\title{
Royal Osteoporosis Society, Osteoporosis Online Conference December 1st 2020: Abstracts
}

\section{Case Report/case series with a learning outcome}

All abstracts marked with ${ }^{\star}$ received commendations.

\author{
Abstract ID: 39 \\ Case Report/case series with a \\ learning outcome \\ Subcategory: Management and treatment \\ Denosumab, an effective therapy for \\ hypercalacamia of sarcoidosis with \\ severe renal impairment
}

\section{R Alshakh, N Gendi}

Department of Rheumatology, Basildon and Thurrock University Hospitals NHS Foundation Trust, Basildon, UK

Background: Denosumab has been used for the management of hypercalcaemia of malignancy where bisphosphonates are contraindicated (e.g. due to severe renal impairment). Unlike bisphosphonates, denosumab is not cleared by the kidneys. In one case report of a multiple myeloma patient with severe renal impairment, denosumab therapy was associated with improvement in renal function. There are limited data for its use in the management of resorption related hypercalcaemia due to other causes including sarcoidosis.

Clinical presentation: A 60 year old female was admitted with hypercalcaemia of $3.7 \mathrm{mmol} / \mathrm{L}$. Comorbidities included chronic kidney disease (CKD), diabetes mellitus with severe retinopa- thy, non-alcoholic fatty liver disease, hypertension, and previous stroke.

Investigation: Parathyroid hormone (PTH) was suppressed at $0.7 \mathrm{pmol} / \mathrm{L}$ (normal range 1.3-9.3) and total 25 vitamin $\mathrm{D}$ was low at $35 \mathrm{nmol} / \mathrm{L}$. CT scan showed splenomegaly and multiple enlarged lymph nodes. Lungs were clear. A CT guided para-aortic lymph node biopsy revealed non-caseating epithelioid granulomas with multinucleated giant cells.

Management: Hypercalcaemia initially responded to intravenous (IV) hydration, high doses of IV and oral glucocorticoids, and IV pamidronate. Azathioprine was later started and titrated. However on reducing the dose of oral prednisolone, serum calcium started to rise reaching $2.9 \mathrm{mmol} / \mathrm{L}$. Her eGFR was $22 \mathrm{~mL} / \mathrm{min}$. Using FRAX, the 10 year probability of major osteoporotic and hip fracture was $13 \%$ and $1.1 \%$ respectively.

Calcium normalised (and renal function improved) following subcutaneous injection of $60 \mathrm{mg}$ of denosumab (D'mab) (see Table). Repeat PTH was $21.3 \mathrm{pmol} / \mathrm{L}$; elevated as would be expected in CKD. She is currently on $4 \mathrm{mg}$ of
Ther Adv Musculoskel Dis 2020, Vol. 12: 1-66 DOI: $10.1177 /$ 1759720X20969289

(c) The Author(s), 2020. Article reuse guidelines: sagepub.com/journalspermissions

\begin{tabular}{|c|c|c|c|c|c|c|c|c|c|}
\hline Date & $13 / 12 / 18$ & $27 / 12 / 18$ & $18 / 01 / 19$ & $19 / 02 / 19$ & March 2019 & $15 / 05 / 19$ & $11 / 06 / 19$ & $16 / 09 / 19$ & $25 / 02 / 2020$ \\
\hline $\begin{array}{l}\text { Adjusted } \\
\text { calcium (2.2- } \\
2.6) \mathrm{mmol} / \mathrm{L}\end{array}$ & 2.85 & 2.7 & 2.68 & 2.91 & \multirow{3}{*}{$\begin{array}{l}\text { D'mab } \\
\text { given }\end{array}$} & 2.6 & 2.48 & 2.42 & 2.43 \\
\hline $\begin{array}{l}\text { Creatinine (45- } \\
\text { 85) } \mu \mathrm{mol} / \mathrm{L}\end{array}$ & - & 245 & 192 & 198 & & 184 & 183 & 167 & 150 \\
\hline Medications & $\begin{array}{l}15 \mathrm{mg} \\
\text { prednioslone } \\
\text { (pred) }\end{array}$ & $\begin{array}{l}10 \mathrm{mg} \\
\text { pred }+ \\
150 \mathrm{mg} \\
\text { AZT }\end{array}$ & $\begin{array}{l}10 \mathrm{mg} \\
\text { pred + } \\
200 \mathrm{mg} \\
\text { AZT }\end{array}$ & $\begin{array}{l}9 \mathrm{mg} \text { pred } \\
+200 \mathrm{mg} \\
\text { AZT }\end{array}$ & & & & $\begin{array}{l}7 \mathrm{mg} \text { pred } \\
+200 \mathrm{mg} \\
\text { AZT }\end{array}$ & $\begin{array}{l}6 \mathrm{mg} \text { pred } \\
+200 \mathrm{mg} \\
\text { AZT }\end{array}$ \\
\hline
\end{tabular}


prednisolone and gradually weaning off. She remains on azathioprine $100 \mathrm{mg} \mathrm{BD}$.

Discussion: We conclude that denosumab could be an option for the management of hypercalcaemia of sarcoidosis if bisphosphonates are contraindicated. More data are required to establish safety at variable degrees of renal impairment and to identify an optimal dose for this indication.

\section{Abstract ID: 74 \\ Case Report/case series with a learning outcome \\ Subcategory: Case Report}

\section{A spontaneous case of oro-antral fistula following single zoledronate administration}

\section{$R$ Dwivedi}

Department of Health care for older people, Queens Medical Centre, Nottingham University Hospitals, Nottingham, UK

Clinical presentation: An 82 year old male with history of T12 fracture, prostate cancer, inflammatory arthritis, polymyalgia rheumatica on maintenance oral steroid (Prednisolone $2 \mathrm{mg}$ odstable dose) was referred to osteoporosis clinic in June 2016 with history of intolerance to oral bisphosphonate.

Investigations: Vitamin $\mathrm{D}=26 \mathrm{nmol} / 1 \mathrm{DEXA}$ scan: T-score of -1.9 (osteopenia) at femoral neck and T12 fracture on spinal morphometry.

Management: There were no prior dental issues. Given prevalent clinical risk factors, following discussion, he received first IV Zoledronic acid infusion in July 2016. His second annual infusion was delayed due to findings of suppressed bone turnover marker (CTX $0.17 \mathrm{ug} / \mathrm{L})$ with a plan to repeat his CTX 6 months later. In the interim, he presented to Maxillofacial (Maxfax) team where he was found to have developed an oro-antral communication (Figure 1) along the socket of the Upper Left premolar (UL5) region, which

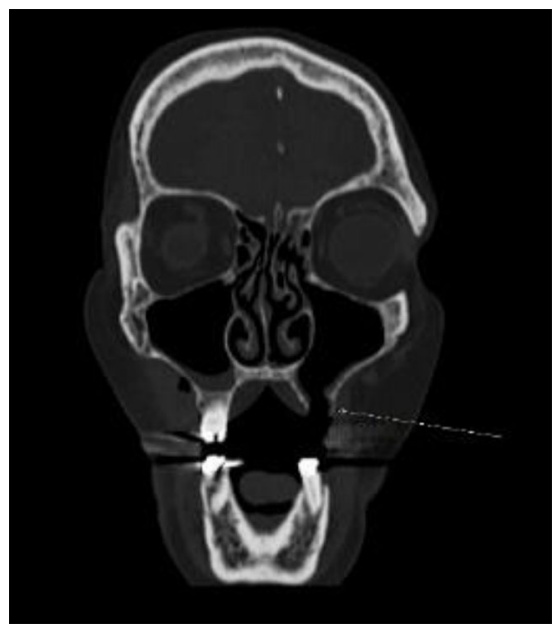

Figure 1.

happened as two adjoining teeth spontaneously came out. He was treated with oral Fluconazole tablets and Nystatin mouth wash and was provided with a cover plate to aid full closure of this oro-antral communication. Further anti-resorptive treatment was discontinued at this point whilst he continued adequate Calcium and vitamin D supplement. As his fistula had considerably shrunk in size at subsequent Maxfax review, he was discharged to community dentists for regular review in June 2019.

Discussion: Oro-antral fistulae are known to occur as sequelae of bisphosphonate-induced osteonecrosis of the jaw (Oladejo O et al. 2010). Risk factors for the development of $\mathrm{ONJ}$ in patients taking anti-resorptive therapy include preexisting dental disease, steroid therapy, smoking and/or alcohol abuse and comorbidities including cancer, with many of these already prevalent in this case. (Khan AA et al. 2015). This case highlights the importance of a through dental assessment in patients with risk factors for ONJ prior to commencement of bisphosphonate. 


\section{Clinical audit/service evaluation}

All abstracts marked with ${ }^{\star}$ received commendations

\section{Abstract ID: 1 \\ Clinical audit/service evaluation Subcategory: DXA}

\section{DXA reporting in men: effect of choice of reference database}

\section{B Roberts ${ }^{1}$, L Blair', S Stewart ${ }^{2}$}

1Osteoporosis and Metabolic Bone Service, Belfast Health and Social Care Trust, Belfast, UK, ${ }^{2}$ DXA Scanning Service, South Eastern Health and Social Care Trust, Belfast, UK

Background: Both the International Society of Clinical Densitometry and WHO recommend that $\mathrm{T}$-scores in both men and women are calculated with reference to a female reference database, as fracture risk correlates better with absolute bone mineral density rather than sexadjusted T-scores. FRAX also states that T-scores should be calculated as above. However anecdotal evidence suggests that most UK centres use sex-specific databases, and 2019 UK guidelines on this are vague, recommending simply that the choice of reference database be by "local agreement".

Objective: To assess the effect of different reference databases on DXA reporting for both GE Lunar and Hologic scanners.

Methods: Fifty male patients' scans, initially reported against a male reference database, were re-analysed against the female equivalent. The change in $\mathrm{T}$-score and bone density classification were noted.

Results: Re-analysis of 25 GE Lunar scans against the female database produced a highly consistent increase of 0.6 in the hip T-score and 0.3 in the spinal $\mathrm{T}$-score. When these patients were classified as osteoporotic, osteopenic or normal, $8 / 25$ men were re-classified at one site and $2 / 25$ at both. For Hologic scans, re-analysis led to an increase in T-score of 0.4 at both hip and spine. Of these 25 patients, six patients were reclassified at one site and one at both. In total, reanalysis of these 50 scans led to an increase in $\mathrm{T}$-score in all cases, and $17(34 \%)$ were given a different "diagnosis" at one or both sites.

Discussion: The choice of reference range for the reporting of male DXA scans makes a very significant change to calculated $\mathrm{T}$-scores and to the proportion of men labelled as osteoporotic or osteopenic. Local variation in this results in overdiagnosis of men with osteoporosis (as defined by WHO), and presumably also wide variation in clinical practice in the management of men with suspected osteoporosis. Using female reference databases for all, as is recommended, would also answer effectively the question of DXA reporting in transgender individuals.

Conclusion: UK authorities should seek to standardise DXA reporting in accordance with scientific reasoning and international guidelines.

\section{Abstract ID: 4 \\ Clinical audit/service evaluation \\ Subcategory: Fracture risk}

\section{An audit: assessing risk of fragility fractures in elderly population and patients on long- term glucocorticoids}

\section{Syed ${ }^{1}$, H Syed ${ }^{2}$, S Quazi ${ }^{1}$}

${ }^{1}$ Barts and The London School of Medicine and Dentistry, Queen Mary University of London, London, UK, ${ }^{2}$ Brighton and Sussex Medical School, University of Sussex, Brighton, UK

Aims and objectives: To determine the adherence of a GP practice to NICE, Bone and Tooth society, National Osteoporosis Society and Royal College of Physicians, guidelines for the risk assessment and prevention of fragility fractures.

Method: This retrospective audit was done using system one to identify all females aged $>65(\mathrm{X})$, all males aged $>75(\mathrm{Y})$ and patients aged $<65$ on repeat prescriptions for oral glucocorticoids ( $Z$ ). Records of patients identified were manually screened to investigate whether they had a fragility fracture assessment since turning 65 for females, and 75 for males. Additionally, for patients $<65$ years on long term glucocorticoids, their records were screened to assess whether they had been referred for DEXA scan as recommended per guidelines.

Relevance/Impact: Falls in the elderly are unfortunately common, with NICE reporting that almost one third of people older than 65 , fall at least once each year. Resulting injuries such as 
fragility fractures can cause significant mortality and both short term and long-term morbidity with a marked decrease in the quality of life. Medical costs from fragility fractures have been estimated to cost NHS England $£ 4.4$ billion each year. These fractures are largely preventable by identifying these high-risk patients and initiating pharmacological or non-pharmacological measures in general practice to reduce the incidence.

Outcomes: Out of 200 patients in group X, only $16 \%$ had records of fragility fracture assessment whereas in patient group Y, only 3\% of had been assessed. Only $30 \%$ of patients identified in group $\mathrm{Y}$ had been referred for DEXA.

Discussion: The lack of awareness and clarity of guidelines have contributed to an inadequate number of patients being assessed for potential risk of fragility fractures. We recommend a presentation outlining the important points from the guidelines and distributing posters created which simplify the guidelines by breaking it down

\section{Abstract ID: 5 \\ Clinical audit/service evaluation Subcategory: DXA}

\section{*Improving DXA scan acquisition: a quality} improvement project

\section{N Powrie' , L Cooper', J Griffin²}

'Department of Radiology, Salford Royal NHS Foundation Trust, Salford, UK, ${ }^{2}$ Royal Osteoporosis Society, Bath, UK

Background: Consistent positioning of patients for Dual Energy X-ray Absorptiometry scans (DXA) is essential for providing accurate and precise measurements of Bone Mineral Density (BMD). Poor positioning can result in artificial increases or decreases in $\mathrm{BMD}$, and an inability to compare serial measurements with confidence. This could result in inappropriate patient management.

Objective: To improve the quality of DXA scan positioning

Methods: A random sample of 25 DXA scans of the spine and hip were selected each month between November 2018 and May 2019 and then evaluated against the following standards, using a standardized audit tool.

Lumbar spine; Does the scan start at L5/S1?; Does the scan terminate at T12?; Is the spine central in the field of view (FOV)?; Is the spine vertical in the FOV?

Hip; Is the femur vertical in the FOV?; Is the acetabulum fully visualised?; Is there little or no lesser trochanter visible (internal rotation)?; Is there at least $5-10 \mathrm{~cm}$ below the ischial tuberosity included?

The percentage of scans meeting each standard (criterion accuracy), the site accuracy (spine and hip) and the overall accuracy was calculated. Areas for improvement identified from the data were regularly fed back to the DXA radiographers during the monthly audit cycle.

Results: Between November 2018 and May 2019 , site accuracy at the spine increased from $65 \%$ to $93 \%$, and at the hip from $76 \%$ to $90 \%$. Overall accuracy increased from $70.5 \%$ to $91.5 \%$. Early trends identified inconsistency in internal rotation of the hip which steadily improved following instruction to DXA staff. The greatest improvements were demonstrated in the correct vertical alignment of both spine and hip scans, and termination of the spine scan at T12.

Discussion: Significant improvements were seen in the overall quality and consistency of DXA positioning, which will improve the reliability and accuracy of BMD measurements at Salford Royal. There is potential for future audit projects to assess the accuracy of scan analysis and positioning in Vertebral Fracture Assessment (VFA) scans using a similar methodology.

Conclusion: This quality improvement project resulted in significant improvements in the quality and consistency of DXA scan positioning

\section{Abstract ID: 6 \\ Clinical audit/service evaluation Subcategory: Management and treatment}

Falls service referral by DXA radiographers improves the identification and appropriate referral of patients at high risk of falls

\section{N Powrie ${ }^{1}$, R Broadbent ${ }^{2}$}

'Department of Radiology, Salford Royal NHS Foundation Trust, Salford, UK, ${ }^{2}$ Intermediate Care Rehabilitation, Salford Royal NHS Foundation Trust, Salford, UK

Background: Falls are common in individuals over 65 and a major cause of fractures. Addressing 
falls risk is essential to osteoporosis management. National Institute of Health and Care Excellence guidelines recommend evaluating falls risk in all patients over 65 . It was routine practice locally to evaluate falls risk when attending for Dual-energy X-ray Absorptiometry (DXA) scans. When appropriate, falls referral was recommended in the DXA report. Baseline audit of a random sample of 50 patients showed only $18 \%$ received a referral. Of those with no previous referral, only $6.8 \%$ received one.

Objective: To evaluate whether falls referral by DXA radiographers improves the proportion of high-risk patients being referred.

Methods: A protocol was developed for falls service referral by DXA radiographers, in line with the local referral pathway. All patients who had experienced one or more falls, reported poor balance or worried about falling, and who consented to referral, were referred by the DXA radiographers.

In a follow up audit, we evaluated; The proportion of patients over 65 who had their falls risk assessed; The proportion of patients at risk of falls offered falls service referral; The proportion of patients who consented to referral who subsequently received one. Data was collected by evaluating standard text in DXA scan reports.

Chi-squared statistics were used to evaluate the probability of change referral rates being due to chance.

Results: We found $96.2 \%$ (359/373) of patients over 65 had falls risk assessed; 99.2\% (246/248) of these were offered falls referral; $91.4 \%(74 / 81)$ of patients who consented to referral received one.

Most (76\%) patients eligible for referral declined this offer.

Compared to the baseline audit, the proportion of patients with no previous referral, who subsequently received one, significantly increased $(6.8-19.4 \%, p=0.044)$.

Discussion: Radiographer led falls referral improved the number of patients appropriately referred. However, most patients declined referral. The reasons for this are unclear; further work is planned. Other reasons for not receiving referrals include patients living out of area, or incomplete evaluations at the DXA appointment.
Conclusion: Falls service referral by DXA radiographers improved the rate of appropriate falls referrals

\section{Abstract ID: 8 \\ Clinical audit/service evaluation Subcategory: Management and treatment}

\section{*Osteoporosis treatment gap in patients at risk of fracture in European primary care: a multi-country cross-sectional study}

EV McCloskey', SE Papapoulos², J Rathi3, M Blagden ${ }^{4}, K$ Palmer ${ }^{5}$, RK Stad ${ }^{6}$, J O'Kelly, $\mathrm{SHeijmans}^{7}$

${ }^{1}$ Oncology and Metabolism, University of Sheffield, Sheffield, UK, ${ }^{2}$ The Leiden Center for Bone Quality, Leiden University Medical Centre, Leiden, Netherlands, ${ }^{3}$ Carrig Medical Centre, Cork, Ireland, ${ }^{4}$ Ashgate Medical Practice, Chesterfield, UK, ${ }^{5}$ Amgen Ltd, Uxbridge, UK, ${ }^{6}$ Amgen Europe $\mathrm{GmbH}$, Rotkrues, Switzerland, ${ }^{7}$ ResearchLink, Linkebeek, Belgium

Introduction: Primary care (PC) physicians are important for the management of osteoporosis (OP). However, recent European data regarding diagnosis and treatment rates of postmenopausal $\mathrm{OP}$ in the PC setting are lacking.

Objective: Assess patterns of real-world OP diagnosis and medical treatment in European PC.

Method: Eligible patients were communitydwelling women aged $\geqslant 70$ visiting their PC physician for any reason were consented to participate in the study. Patient information were collected via self-reported questionnaires and medical records. The primary outcome of the study was the proportion of women aged $\geqslant 70$ years at increased risk of fragility fracture $(\mathrm{FF})$ who were not receiving OP medication (the "treatment gap"). Increased risk of FF was defined as at least one (1) history of fracture, (2) 10-year probability of both hip and major OP fracture above countryspecific FRAX thresholds, (3) T-score $\leqslant-2.5$.

Results: Patients were enrolled between March and October 2018 from eight countries (Belgium, France, Germany, Ireland, Poland, Slovakia, UK and Switzerland). 2077 women (55\%) met at least one definition for increased risk of fracture: 1200 women with a prior fracture and 318 with a $\mathrm{T}$-score $\leqslant-2.5$. Three quarters of women at increased risk of FF were not receiving any OP medication [1550/2077; $75 \% \quad(95 \%$ CI: $73-$ $77 \%)]$. The treatment gap was lower in women who had a diagnosis of OP [31\% (198/641)] than those not diagnosed with OP [94\% (1318/1401)]. 
There were variations between countries in the proportions of patients at increased risk of fracture (from $41 \%$ to $76 \%$ ) and in the proportions diagnosed with OP (from $15 \%$ to $30 \%$ ). The treatment gap was over $50 \%$ in all countries and less in patents with a diagnosis of (by 29-50\%). Types of OP medication used in the different countries were consistent with local reimbursement guidelines.

Conclusions: This study of OP management in European PC found a large treatment gap amongst women aged $\geqslant 70$ who are at increased risk of FF. In the UK, insufficient OP diagnosis appears to be an important barrier to treatment.

\section{Acknowledgements}

Study was sponsored by Amgen

\section{Abstract ID: 9 \\ Clinical audit/service evaluation Subcategory: DXA}

\section{Non-attendance in individuals referred for DXA scan when identified by a fracture Liaison Service}

TC Clark', SAY Young-Min', MF Finnegan', JP Penketh', LH Hamilton', BRM RodgersMansfield', MA Allen', SD Day', RMD MurdayBailey', NH Hoggett'2, SS Spicer', AP Pritt², CB Beevor ${ }^{1}$, MHE Edwards ${ }^{1}$

${ }^{1}$ Rheumatology, Portsmouth NHS Trust, Portsmouth, UK, ${ }^{2}$ Medical Physics, Portsmouth NHS Trust, Portsmouth, UK

Background: Fracture Liaison Services (FLS) have been developed to close the secondary fracture prevention gap. In younger individuals this assessment often requires a DXA scan; we found that a proportion of patients were not attending for their DXA scans when arranged. This has economic implications to the service and could also have adverse outcomes for patients.

Objective: To assess impact of reminder telephone call on attendance for DXA following initial non-attendance.

Methods: Between July 2014 and March 2018 we identified all individuals who did not attend for a DXA scan following referral by our FLS. Having found high rates of non-attendance in those offered a further appointment; we instituted a reminder telephone call prior to the subsequent DXA appointment. Thereafter between April 2018 and
November 2019, we reassessed rates of attendance for the 1st and 2nd DXA appointments.

Results: Between April 2018 and November 2019, 1681 patients were referred by the FLS for a DXA scan. Of these (8\%) did not attend for their first appointment (4\% improvement from 2014 to 2018 ). When a second appointment was offered $(44 \%)$ attended for this (34\% improvement) $46 \%$ did not attend and $10 \%$ were not offered a second appointment. Of the 59 patients who attended their second offered DXA appointment $(37 \%)$ were treated.

Conclusion: It has been noted that there has been a small improvement in the number of patients who attended for their first DXA appointment. There has been a large improvement of $34 \%$ in the patients who attended when given a second DXA appointment $37 \%$ of patients were treated after attending their second DXA scan showing that this group is at significant risk and often require treatment after assessment. Over the last 2 years there has been improvement in the attendance of patients for their DXA scans and the FLS need to maintain this improvement to help reduce the risk of further fragility fractures. The reason for this improvement has been attributed to the DXA department making a reminder phone call the day before appointments.

\section{Abstract ID: 11 \\ Clinical audit/service evaluation Subcategory: Hip fracture}

\section{Best foot forwards: balancing the risks of suffering a hip fracture}

\section{AJ Johanasen', DI Inman', LF Fagan', TB Bunning'2, SH Hawley ${ }^{3}$, AJ Judge ${ }^{3}$}

${ }^{1}$ National Hip Fracture Database (NHFD), Royal College of Physicians, London, UK, ${ }^{2}$ Crown Informatics, Nottinghamshire, UK, ${ }^{3}$ NDORMS, University of Oxford, Oxford, UK

Introduction: Staff on acute trauma wards will be familiar with the observation that patients with a history of stroke usually present with hip fractures affecting their weaker side. This reflects poorer bone density in the paretic limb, ${ }^{1}$ and a greater tendency to fall in that direction. We explored whether there might be a tendency for patients to fall towards their non-dominant side, as reflected in an increased likelihood of presenting with hip fractures on this side. 
Methods: Since 2007 the NHFD has collected data for all patients presenting in England, Wales and Northern Ireland. We analysed data for the 133,789 people who presented during the 2018 and 2019 calendar years, looking at the side and type of fracture recorded for each individual.

Results: We found larger numbers of fractures affected the left hip; 69,775 (52.2\%) compared with just $64,014(47.8 \%)$ affecting the right. This pattern was consistent across the different types of fracture with which these patients presented, and the types of surgery subsequently needed. NHFD data from previous years confirmed that this pattern was consistent within a cohort that now exceeds 500,000 cases.

Conclusions: The NHFD does not record the handedness of patients, but the direction of our finding suggests right hand (and foot) dominance of the population; this might have been more marked if the $9.2 \%$ of left-handed ${ }^{2}$ patients were excluded. In a post-Brexit era, we should not comment on the clinical significance of this $52 \%$ versus $48 \%$ difference, but it is unlikely that clinicians in individual units or even researchers running multicentre trials would have noted or been powered to prove this statistically significant finding. The results may however interest those studying balance and falls prevention who will know the handed-ness of individual patients.

\section{References}

1. Johansen A, Harris W and Stone M. Ultrasound assessment of bone: hemiparesis following stroke as a model for immobilisation osteoporosis. Arch Gerontol Geriatr 1997; 25: 299-304.

2. Papadatou-Pastou M, Panagiotidou DA, Abbondanza F, et al. Hand preference and mathematical learning difficulties: new data from Greece, the United Kingdom, and Germany and two meta-analyses of the literature. Epub ahead of print May 2019. DOI: 10.31234/osf.io/pc6zh.

\begin{tabular}{|lr|rr|rr|}
\hline Type of hip fracture & Total & \multicolumn{2}{|c|}{ Left } & \multicolumn{2}{|c|}{ Right } \\
Intertrochanteric - grade A1/A2 & 42645 & 22054 & $51.7 \%$ & 20591 & $48.3 \%$ \\
Intertrochanteric - grade A3 & 5310 & 2769 & $52.1 \%$ & 2541 & $47.9 \%$ \\
Intracapsular - displaced & 67631 & 35713 & $52.8 \%$ & 31918 & $47.2 \%$ \\
Intracapsular - undisplaced & 11009 & 5607 & $50.9 \%$ & 5402 & $49.1 \%$ \\
Subtrochanteric & 7033 & 3544 & $50.4 \%$ & 3489 & $49.6 \%$ \\
Missing & 161 & 88 & $54.7 \%$ & 73 & $45.3 \%$ \\
Totals & 133789 & 69775 & $\mathbf{5 2 . 2} \%$ & 64014 & $47.8 \%$ \\
\hline
\end{tabular}

Figure 1.

\section{Abstract ID: 12 \\ Clinical audit/service evaluation \\ Subcategory: Management and treatment}

\section{${ }^{*}$ Evaluation of treatment target response in real-world clinical service}

\author{
DE Powell, SF Evans, C Rakieh \\ Metabolic Bone Health Service, Robert Jones \& Agnes Hunt Orthopaedic
} Hospital NHS Foundation Trust, Oswestry, UK

Background: In real-world clinical services pretreatment levels of bone turnover markers are not always available to assess response. A target response in the lower half of the premenopausal reference range has been suggested. However, some subjects may have been within target prior to treatment.

Objective: To evaluate the number of subjects within the treatment target prior to treatment. To evaluate suppression of bone turnover markers in response to bisphosphonate in a real-world clinical osteoporosis service relative to previously published reference intervals.

Methods: Intact amino-terminal propeptide of type I procollagen (PINP) and urinary $\mathrm{N}$-telopeptide crosslink of type I collagen (uNTx) levels were measured in postmenopausal women attending a routine Metabolic Outpatient appointment, 46 treatment naïve subjects (TN), 41 on alendronate (ALN) and 28 on IV Zoledronate (ZOL). Non-fasting PINP was measured using the automated i-SYS autoanalyser and UNTx by ELISA corrected for creatinine levels.

Results: There was no difference in age, height, weight or BMI between the groups. Both PINP and UNTx were significantly higher in the TN group $(p<0.001)$. ZOL suppressed both markers to a greater extent than ALN $(p=0.002)$. The majority of subjects were above the premenopausal mean in the TN group, $87 \%$ for PINP and $82 \%$ for uNTx. Only $2(7 \%)$ ZOL subjects had a PINP level above the premenopausal mean compared to $19(46 \%)$ of ALN subject $(p<0.001)$ for $\mathrm{UNTx}$ the values were $6 \%$ for $\mathrm{ZOL}$ and $35 \%$ for ALN $(p<0.05)$. There was no difference in the proportion of subjects with a result in the lower half of the premenopausal reference interval for PINP compared to UNTX in either the ZOL or ALN groups.

Discussion: Less than $20 \%$ of subjects had a marker level in the lower half of the premenopausal reference range prior to the initiation of therapy. Subjects on ZOL were more likely to achieve 
this target in comparison to ALN. No significant difference between the number of responders measured by PINP or uNTx.

Conclusion: For the majority of subjects without a pre-treatment marker level the lower of the premenopausal reference range is a useful target to determine if there is a response to treatment.

\section{Abstract ID: 13 \\ Clinical audit/service evaluation Subcategory: FLS \\ ${ }^{*}$ Fracture liaison service: audit of telephone medication reviews}

DA Nelson, LAL Robertson, MW French

Bone Metabolism Unit, NHS West Glasgow ACH, Glasgow, Scotland, UK

Background: Osteoporotic fractures cause significant morbidity, mortality and cost. Antiosteoporosis treatments reduce fracture risk but many high-risk individuals remain undiagnosed or untreated. In an established FLS the next priority is to address the Achilles' Heel of osteoporosis care - sub-optimal medication adherence. Contact with healthcare professionals has the potential to support adherence and is integral to the Royal Osteoporosis Society Clinical Standards for FLS. Presented here is audit data from telephone medication reviews with patients who attended the FLS clinic and had a new oral bisphosphonate (OBP) recommendation between January and December 2019.

Objective: To ascertain the extent to which patients were correctly taking recommended OBP, identify and address barriers to adherence.

Methods: FLS Nurses telephoned eligible patients 3 months after OPB recommendation to determine if treatment was commenced, continued, tolerated and taken correctly. Information on new falls or fractures was captured, as was qualitative data reflecting patients' perspective regarding telephone contact.

Results: 144 patients with a new OBP were identified (in clinic, $100 \%$ consented to contact). Of 115 patients contacted by telephone, 74 reported no issues. Of the remainder: 10 declined OBP (five agreed to revisit decision with sooner review); 14 delayed initiating OBP; 12 were taking OBP incorrectly; eight were intolerant of initial OBP (3 switched to an alterative OBP and five were referred for parenteral treatment). Falls prevention or exercise referrals were made for five patients. 18 patients, un-contactable by telephone, were sent a letter inviting them to contact the FLS. 11 had contacted FLS before scheduled follow-up.

Discussion: Consistent with published literature, at 3 months, we found $64 \%$ reported adherence whilst $31 \%$ were non-adherent (taking OBP incorrectly or not at all). Telephone review provided an effective and well-received opportunity to offer additional support and drew consistently positive feedback. This data reflects the first stage of a service development (without additional resources) so began with FLS clinic attenders only. Building on this success, follow-up at 3- and 12-month will be offered to all FLS patients recommended OBP.

Conclusion: This intervention identified $31 \%$ non-adherence and provided the opportunity to improve this.

\section{Abstract ID: 16 \\ Clinical audit/service evaluation Subcategory: Management and treatment \\ Experience of management of chronic kidney disease-mineral and bone disorder at a district general hospital, UK}

\section{RN Alshakh, A Nandagudi}

Rheumatology, Basildon and Thurrock University Hospitals NHS Foundation Trust, Basildon, UK

Background: Abnormal bone turnover or strength is one of the manifestations of chronic kidney disease- mineral and bone disorder (CKDMBD) with subsequent increase in fracture risk. With limited evidence, the management is challenging. In 2016, we developed a local pathway in collaboration with the nephrologists. KDIGO 2009 guidelines recommend consideration of bone biopsy. Due to limited access and the associated risks with this invasive procedure, we agreed to use bone ALP as an indicator of bone turnover state to guide antiresorptive therapy. In CKD stage 4 , we suggest denosumab if there is evidence of high bone turnover. For patients with stage $5 \mathrm{CKD}$, optimisation of the biochemical abnormalities is advised.

Objective: Assess the efficacy of the pathway as per KDIGO standards. 
Methods: Medical records of all CKD-MBD patients were retrospectively reviewed.

Results: A total of 32 patients were seen, 25 $(78 \%)$ were women. All patients were Caucasian and over 50 years of age. Oral bisphosphonates had been first line therapy in $47 \%$. Only four patients $(13 \%)$ were on dialysis. Of the remaining $88 \%$ (28 patients), $64 \%$ (18) had fragility fractures (50\% with osteopenia only), while $36 \%$ had low BMD without fractures.

Six patients (19\%) had stage $5 \mathrm{CKD}$. Amongst the ckd stage four patients, $44 \%$ received denosumab (1-4 doses), 9\% are awaiting first dose, $9 \%$ declined denosumab, $9 \%$ had evidence of low bone turnover, $6 \%$ died, and one patient was discharged due to repeated failure to attend clinic. None of the patients had fragility fractures after commencing therapy.

Discussion: Literature suggests that symptoms and signs of CKD-MBD including fractures do not occur until patients are on dialysis. The majority $(88 \%)$ of patients in this cohort were not on dialysis. Of those, $64 \%$ had fragility fractures. There was osteopenia in $50 \%$ of those who had fractures.

Conclusion: This is a small sample size but the absence of fragility fractures or adverse effects with treatment is encouraging. We aim to reassess in a few years with DXA for more long term data.

\section{Abstract ID: 18 \\ Clinical audit/service evaluation Subcategory: Hip fracture}

${ }^{*}$ Hip fracture incidence is decreasing, but are men being left behind?

\section{AJ Johansen', DI Inman', LF Liz Fagan', TB Bunning ${ }^{2}$, SH Hawley ${ }^{3}$, AJ Judge ${ }^{3}$ \\ 'National Hip Fracture Database (NHFD), Royal College of Physicians, London, UK, ${ }^{2}$ Crown Informatics, Nottinghamshire, UK, ${ }^{3}$ NDORMS, University of Oxford, Oxford, UK}

Introduction: Hip fracture typically affects the oldest age groups - the same age groups that are expanding most dramatically in number with current demographic trends. This might lead the unwary to predict a dramatic increase in hip fracture numbers, ${ }^{1}$ though data from many countries have suggested otherwise. ${ }^{2}$ We examined trends in hip fracture incidence using data

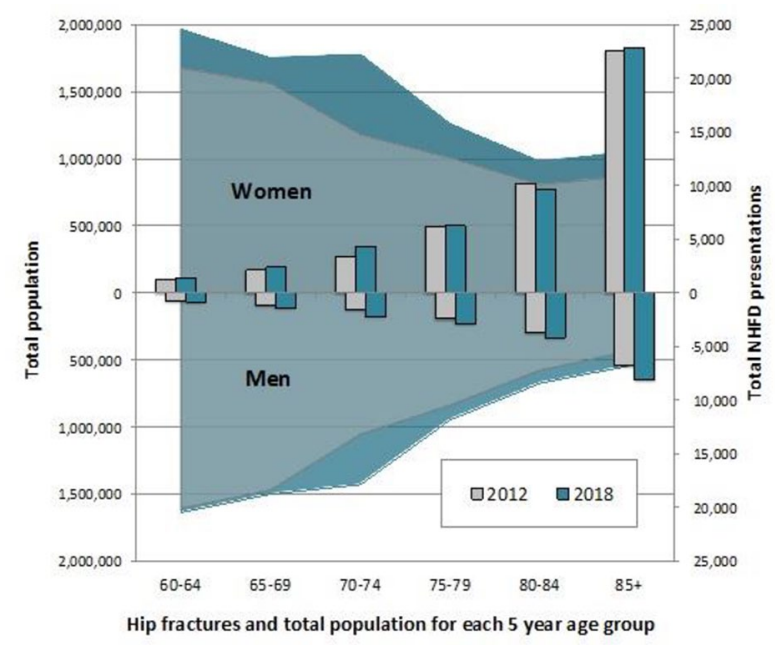

Figure 1.

on presentations recorded by the National Hip Fracture Database (NHFD).

Methods: Since 2007 the NHFD has collected data for all patients presenting in England, Wales and Northern Ireland. We examined NHFD data from the 2012 calendar year (when case ascertainment first reached its current 95\% figure) and drew comparison with equivalent 2018 data; the most recent year for which ONS figures for the population of these three countries are available.

Results: ONS data show the over 60-year-old population to have increased by $18.5 \%$ between 2012 and 2018, but we found total hip fracture presentations had only increased by $7.1 \%$. This might suggest a decrease in age-specific hip fracture incidence due to general improvements in older people's health. However, we found a difference between the two sexes. The proportion of hip fractures affecting men rose from $26.3 \%$ to $29.4 \%$. Per 1000 over 60 -year-olds, hip fracture incidence fell in women by $17 \%$, from 6.5 to 5.3 $(p<0.001)$, which contrasts to a $7 \%$ rise from 2.7 to 2.9 in men $(p<0.001)$.

Conclusions: Improving public awareness about the impact of osteoporosis in women may have contributed to this difference and suggests greater attention should be paid to the importance of hip fracture prevention in men; especially important since the outcome of hip fracture is so much poorer in men; in 2018, $10.5 \%$ of men died during their hospital admission, nearly twice the $5.7 \%$ figure for women (NHFD). 


\section{References}

1. Burge R, Worley D, Johansen A, et al. The cost of osteoporotic fractures in the United Kingdom: projections for 2000-2020. I Medical Economics 2001; 4: 51-62.

2. Cooper C, Cole ZA, Holroyd CR, et al. Secular trends in the incidence of hip and other osteoporotic fractures. Osteoporos Int 2011;22: 1277-1288.

\section{Abstract ID: 24 \\ Clinical audit/service evaluation Subcategory: FLS}

\section{4 and 12 month treatment adherence checks: best use of precious resources?}

MS Jamal', J Shipley', D Hart', S Warren', J Webb' ${ }^{1}, C_{\text {Gregson 2,3, S Hardcastle1, T Ahmed }}$

${ }^{1}$ Rheumatology, Royal United Hospital, Bath, UK, ${ }^{2}$ Geriatrics, Royal United Hospital, Bath, UK, ${ }^{3}$ Musculoskeletal Research Unit, University of Bristol, Bristol, UK

Background: Adherence with bone protection treatment is a common challenge worldwide. UK national clinical standards recommend assessing adherence at 4 and 12 months post-fracture. In our unit we routinely assess adherence at 12 months post-fracture via postal questionnaire.

Objective: To evaluate if additional adherence checks at 4 months improve treatment adherence at 12 months.

Methods: Our FLS identified patients between March 2017 and August 2018 across three clinical commissioning groups (CCGs). Adherence questionnaires were sent to Somerset CCG patients at 4 and 12 months and to BANES and Wiltshire CCG patients at 12 months only. Data were collected through our locally-held FLS database.

Results: 2592 patients were analysed.192 fractured a hip and 256 a vertebrae. Treatment to reduce fracture risk was recommended in 1510 $(58.3 \%)$ patients. See Figure 1: we found no evidence that an adherence questionnaire at 4 months improved reported adherence by 12 months in Somerset $(28 \%$ versus $35 \%$; $p=0.116$ ), with no difference in reported adherence by 12 months between the two CCG groupings $(31 \%$ versus $35 \%$; $p=0.315)$.

Discussion: Somerset patients improved adherence by $7 \%$ in absolute terms between 4 and 12 months. If we replicated this improvement in BANES/Wiltshire, this would have resulted in an additional 91 patients reporting adherence. If these patients all had a 10-year probability of hip fracture of 5\% and major osteoporotic fracture of $20 \%$, we might prevent an additional 2 hip and 7 peripheral fractures over 10 years.

Conclusion: An additional questionnaire-based adherence check at 4 months appears to lead to a minimal (non-significant) improvement in reported adherence. Telephone follow-up may be more effective but requires much greater resource. Focusing on improving fracture identification may be a better use of limited resources than instituting additional adherence checks.

\section{Abstract ID: 31 \\ Clinical audit/service evaluation Subcategory: Vertebral fracture}

*Incidental vertebral fractures on radiographic imaging: a novel radiologist initiated rapid referral pathway to the fracture liaison service

\section{MDJ Gibson, AL Dolan}

Department of Rheumatology, Queen Elizabeth Hospital, Lewisham and Greenwich NHS Trust, London, UK

Introduction: The presence of vertebral fractures is known to be a powerful predictor for

\begin{tabular}{|c|c|c|c|c|c|}
\hline CCG & $\begin{array}{l}\text { Patients with a } \\
\text { fracture (n) }\end{array}$ & $\begin{array}{c}\text { Treatment } \\
\text { recommended } \\
(\%)\end{array}$ & $\begin{array}{c}\text { Questionnaire } \\
\text { responses } 4 \text { \& } \\
12 \text { months } \\
(\%)\end{array}$ & $\begin{array}{l}\text { Adherence } \\
\text { reported at } \\
4 \text { months }\end{array}$ & $\begin{array}{l}\text { Adherence } \\
\text { reported at } 12 \\
\text { months }\end{array}$ \\
\hline BANES/Wilts & 2238 & $1298(58 \%)$ & $\begin{array}{c}\mathrm{n} / \mathrm{a} \text { at } 4 \mathrm{~m} \\
797(61 \%) \text { at } \\
12 \mathrm{~m}\end{array}$ & $\mathrm{n} / \mathrm{a}$ & $\begin{array}{c}408 / 1298 \\
(31 \%)\end{array}$ \\
\hline Somerset & 354 & $212(60 \%)$ & $\begin{array}{c}133(63 \%) \text { at } \\
4 \mathrm{~m} \\
130(61 \%) \text { at } \\
12 \mathrm{~m}\end{array}$ & $\begin{array}{l}59 / 212 \\
(28 \%)\end{array}$ & $74 / 212(35 \%)$ \\
\hline
\end{tabular}

Figure 1. 
future hip fractures but the recent $\mathrm{RCR} / \mathrm{RCP} /$ ROS national audit on radiology reporting of osteoporotic vertebral fragility fractures found that underdiagnosis of vertebral fractures remains widespread. In response, our unit implemented a radiology initiated referral pathway to the fracture liaison service (FLS) following the incidental finding of vertebral fracture on imaging.

Methods: We incorporated a FLS referral button into the radiology reporting system by adapting a pre-existing process used to generate oncology referrals. Patient's were subsequently invited to attend for bone mineral density measurement and estimation of fracture risk by FRAX. Referrals between October 2019 and January 2020 were audited. We measured patient demographics \& characteristics, osteoporotic risk fractures, bone mineral density and treatment outcomes. FRAX-10-year fracture risk was compared pre and post vertebral fracture identification (two-tailed paired $t$-test with significance threshold $p<0.05$ ).

Results: 40 patients were referred [63\% female $(n=25)$, median age 77 years (range: $39-$ 91 years)]. A total of 63 vertebral fractures were identified on imaging (CT: $47.5 \%(n=19)$, MRI: $12.5 \%(n=5)$, XR: $40 \%(n=16))$. Fracture severity: Grade I: $25 \%(n=10)$, Grade 2: 35\% $(n=14)$, Grade 3: $40 \% \quad(n=16)$. WHO classification: Normal 7.5\% $(n=3)$, Osteopenia $42.5 \%(n=17)$, Osteoporosis $50 \%(n=20) .72 .5 \%$ had no prior history of fracture $(n=29) .67 .5 \%$ had not previously received treatment for osteoporosis. FRAX 10 year risk of major osteoporotic fracture $(p<0.0001)$ and hip fracture $(p<0.0001)$ differed significantly following identification of vertebral fracture. Bisphosphonate therapy was advocated in $82.5 \%$ (osteoporosis $n=20$, osteopenia $n=13) .45 \%$ of patients $(n=18)$ were referred to osteoporosis specialist clinic for consideration of parenteral therapy.

Conclusions: This novel referral pathway is an inexpensive, easy to implement and high-yield method of identifying patient's at high risk of future osteoporotic fracture. The presence of an incidental vertebral fracture resulted in significantly higher estimation of future fracture risk and frequently resulted in the recommendation to commence bisphosphonate therapy even in those with osteopenic bone density.

\section{Abstract ID: 33 \\ Clinical audit/service evaluation \\ Subcategory: Management and treatment}

\section{*Osteoporosis management when denosumab therapy ends: an audit of current practice}

\author{
J Zhang ${ }^{1}$, MA Clynes ${ }^{1}$, C Cooper ${ }^{1,2}$, \\ EM Dennison ${ }^{1,3}$
}

${ }^{1}$ MRC LEU, Southampton, UK, ${ }^{2}$ NIHR Musculoskeletal Biomedical Research Unit, University of Oxford, Oxford, UK, ${ }^{3}$ Victoria University of Wellington, Wellington, New Zealand

Introduction: In recent years denosumab therapy has been widely used in the treatment of osteoporosis. However, since its first introduction to clinical practice, evidence has demonstrated discontinuation leads to rebound bone loss and risk of vertebral fracture. In view of this, guidance has been updated recommending transition to an alternative osteoporosis therapy. Given that osteoporosis management is largely managed in primary care, we performed this audit to investigate whether this educational message was being effectively relayed to primary care clinicians.

Methods: In this study we closed the audit loop of a previous study of the first 50 patients commenced on denosumab at University Hospital Southampton in 2013; we explored the percentage of patients remaining on denosumab 6 years later, using the hospital electronic system, which is linked to the general practice electronic system, to confirm prescriptions for osteoporosis therapy. In cases where it was unclear, letters were written or telephone calls were made to general practices for clarification. Continuation of denosumab or use of an alternative agent was then recorded.

Results: Nineteen (38\%) patients had died since the initial audit, reflecting the use of the agent in later life post hip fracture. Of 15 patients no longer on denosumab, $9(60 \%)$ were found not to be on alternative bone protection without other information recorded, 1 (7\%) had denosumab suspended by the GP (with the presumption it may be restarted at a later date), 1 (7\%) had denosumab suspended due to recurrent cellulitis with the view to reassess in a year and 3 $(20 \%)$ were on alternative therapy. One patient was recorded as being on a 'drug holiday'. Of 16 patients remaining on denosumab, $13(81 \%)$ had pre-injection calcium level checks, and 12 
(75\%) had a recorded pre-injection renal function check.

Conclusion: These data reflect inappropriate widespread discontinuation of denosumab without follow-on with an alternative osteoporosis therapy, suggesting that targeted education of primary care physicians is necessary. A high proportion of patients on denosumab are having the relevant blood tests in primary care as per clinical guidelines.

\section{Abstract ID: 35 \\ Clinical audit/service evaluation Subcategory: Management and treatment \\ Outcomes of delayed denosumab retreatment on bone mineral density and fragility fractures: results from an audit of prescribers' adherence to guideline}

JE McLorinan, MY Md Yusof, K Nadesalingam Rheumatology, Bradford Teaching Hospitals NHS Foundation Trust, Bradford, UK

Background: Denosumab increases bone mineral density (BMD) and reduces bone turnover markers for up to 6 months post-treatment. Hence, adherence to timely retreatment is fundamental. Locally, denosumab is prescribed 6 -monthly $(+/-10$ days) in primary care following review and approval by rheumatologists.

Objective: To audit prescribers' adherence and assess outcomes of delayed retreatment with respect to BMD and fragility fractures.

Methods: We conducted a retrospective audit of the first 115 consecutive patients treated with denosumab over a 4-year period. Data was gathered from SystmOne electronic record. DEXA scan results and the presence of fragility fractures pre- and post-therapy were reviewed.

Results: 87/115 patients provided consent and were included in the audit [mean age was 76.1 years; 81 (93\%) were female; $42(48 \%)$ had other rheumatic diseases: $\mathrm{RA}=26, \mathrm{PMR} /$ $\mathrm{GCA}=7, \mathrm{SLE}=3$, Sjogrens $=2$, polymyositis $=2$, others $=2 ; 66(76 \%)$ had prior fragility fractures]. $78 / 87(89 \%)$ had $\geqslant 1$ retreatment with denosumab. Of these, only $35 / 78(45 \%)$ had all their injections on time. 32/87 (36\%) had pre- and post-denosumab DEXA scans, with a mean gap of 42 months between scans. These patients were divided into $100 \%$ adherence $(n=12)$ versus any treatment delay $(n=20)$ groups. Median (IQR) percentage change in BMD total hip was +1 $(-1.4$ to 4.1$) \%$ and $+0.3(-4.7$ to 2.0$) \%$ respectively; $p=0.182$. Fragility fractures following denosumab were recorded in 5/87 (6\%) patients (vertebral $=2$, neck of femur $=1$, pubic ramus $=1$, wrist $=2)$. Of these, $3 / 5(60 \%)$ had at least one treatment delay.

Discussion: More than half of the patients in this audit had delays in denosumab treatment. BMD increased with treatment. Where there were delays there was a trend to a lower degree of improvement in BMD as well as increased rate of fragility fractures, though a formal relationship cannot be deduced.

Conclusion: Measures are required to improve prescriber adherence with the denosumab regimen. Recommendations include better communication with GPs through presentation at training days and utilisation of medication alert systems. Re-audit is in progress following educational events.

\section{Abstract ID: 38 \\ Clinical audit/service evaluation Subcategory: Management and treatment}

*Denosumab adherence rates in a community specialist denosumab clinic

N Dale ${ }^{1}$, F Crawford-Manning 1,2, E Adams', M Tian-Clarke', T Malpass', A Twemlow', K Gould 1 , Z Paskins ${ }^{1,2}$

'Osteoporosis Service, Midlands Partnership NHS Foundation Trust, Haywood Hospital, Stoke-on-Trent, UK, ${ }^{2}$ School of Primary, Community and Social Care, Keele University, Staffordshire, UK

Background: Recent evidence indicates that in even the 'best' GP practices, many patients on denosumab are not having injections on time indicating suboptimal adherence to denosumab injections in primary care. At the local community hospital in 2019 we instigated a community denosumab service; injections are delivered by a member of the secondary care specialist team at community locations close to patient's homes.

Objective: To assess adherence to denosumab injections in a community hospital osteoporosis service.

Methods: 160 patients were who had baseline treatment during the months of January-June 
2019 were identified from department records. Subsequent treatment was recorded as (i) within 4 weeks of due date, (ii) within 8 weeks of due date, (iii) not given within 8 weeks of due date.

Results: Of the 160 patients audited all patients received their follow-up denosumab injection, $91 \%(142 / 160)$ of these were within 4 weeks of the due date. A further $9 / 160$ (5.8\%) received their injection within 8 weeks, and another $9 / 160$ $(5.8 \%)$ outside of 8 weeks. Of those who had delayed treatment (4weeks + of due date) $27.8 \%$ $(5 / 18)$ were due to patient reasons (cancelations or DNA), and $66.7 \%(12 / 18)$ were due to contraindications or comorbidity issues.

Discussion: Adherence to denosumab injections in a community specialist service is excellent and illustrates the value of maintaining these injections in secondary care. Further audit and evaluation would be helpful to explore other clinical and patient outcomes such as monitoring and follow up, fracture rates and satisfaction.

\section{Abstract ID: 40 \\ Clinical audit/service evaluation Subcategory: DXA \\ *UK multi center dual energy $x$-ray absorptiometry scan reporting audit}

\section{JGL Griffin', S Mighton'², KAtkinson', M Cunningham ${ }^{4}, R$ Pettit ${ }^{5}$, A Phair 6 , $R$ Whittleworth ${ }^{7}$}

1Professional Development, Royal Osteoporosis Society, Bath, UK, ${ }^{2}$ Radiology, NHS Western Isles, Stornoway, UK, ${ }^{3}$ Radiology, Leeds Teaching Hospital, Leeds, UK, ${ }^{4}$ Radiology, South Tyrone Hospital, NI, UK, ${ }^{5}$ Medical Physics, Cardiff and Vale UHB, Cardiff, UK, ${ }^{6}$ Radiology, South West Acute Hospital, NI, UK, ${ }^{7}$ Radiology, Llandudno General Hospital, Llandudno, UK

Background: The clinical evaluation (reporting) of diagnostic imaging studies involving ionising radiation is mandated under the ionising radiation (medical exposure) regulations 2017 (IR(ME)R $)^{1}$ and subject to standards set by the Royal College of Radiologists (RCR). ${ }^{2}$ Inconsistencies in the reporting of dual energy x-ray absorptiometry scans (DXA) have been observed through peer review processes and networking events such as the Royal Osteoporosis Society (ROS) National Training Scheme for Bone Densitometry. To support quality improvements in the provision of
DXA services, and secondary fracture prevention services such as fracture liaison services (FLS) in achieving national targets for fractureosteoporosis treatment times, the ROS published a set of standards for reporting of DXA scans in $2019 .^{3}$

Methods: This audit evaluated current DXA reporting practices across 6 UK NHS DXA scanning services, for compliance to the ROS published reporting standards and included 300 reports authored by $>6$ reporters. The audit interrogated each standard through one or more question resulting in some granularity of response for further evaluation.

Overall Results: Standard 1 (report within 3 weeks): $65 \%$. Standard 2 (training and IR(ME) $\mathrm{R}$ entitlement): $76 \%$. Standard 3 (quality assurance and clinical governance) $34 \%$. Standard 4 (local protocol) 54\%. Standard 5 (vertebral fracture assessments) $0 \%$ Standard, 6 (management advice and access to support) $79 \%$.

Discussion: The audit demonstrated variability across all the reporting standards. The area's most commonly non-compliant were around specialist training in DXA reporting, local protocols, quality audit programmes and clinical governance of DXA reporting. The audit also demonstrated variability across sites in achieving the standards with average compliance of $59 \%$, ranging from $23 \%$ to $89 \%$ across all 6 standards. A further audit is planned following quality improvement interventions, which include postgraduate training for the reporting of DXA scans, at all services.

This project is supported by an educational grant by UCB administered by the ROS.

\section{References}

1. Department of Health and Social Care. The ionising radiation (medical exposure) regulations 2017. 2018.

2. Royal College of Radiologists. Standards for interpretation and reporting of imaging investigations. 2nd ed. London: Royal College of Radiologists, 2018.

3. Royal Osteoporosis Society. Reporting dual energy $X$-ray absorptiometry scans in adult fracture risk assessment: Standards for quality. London: Royal Osteoporosis Society, 2019. 


\section{Abstract ID: 41 \\ Clinical audit/service evaluation \\ Subcategory: Management and treatment \\ Pain assessment and management following hip fracture: superior analgesia for the cognitively intact}

\author{
D Muir, A Turk, W Havelock, A Johansen \\ Trauma Unit, University Hospital of Wales, Cardiff, UK
}

Background: Pain management following hip fracture is challenging, as uncontrolled pain and injudicious opioid analgesia can both precipitate delirium. At the 2014 and 2016 NOS conferences we demonstrated that people with cognitive impairment received less opioid analgesia than those with intact cognition. Our quality improvement work has since focused on two new strategies: (a) patients are given individualised doses of morphine or oxycodone at breakfast and lunchtime, in anticipation of physiotherapy, (b) patients' mental state and experience of pain on hip movement is examined on daily rounds, and their analgesia adjusted accordingly.

Objective: To review our approach to pain management after hip fracture surgery.

Method: During February to March 2020, we assessed sequential patients admitted to our orthogeriatric wards. Patients were interviewed 3 days after hip fracture surgery to self-assess their pain on rest and movement. Opioid analgesic use was calculated as an 'oral morphine equivalent dose' over the previous 24-hours.

Results: We assessed 31 patients; 12 (35.5\%) with cognitive impairment (Abbreviated Mental Test $\leqslant 7 / 10$ ). The day- 3 oral morphine equivalent dose was higher in cognitively intact patients than for those with cognitive impairment (18.6 mg versus $11.5 \mathrm{mg}$; $p=0.07$ ). Both figures are higher than those we reported in 2016 (12.5 $\mathrm{mg}$ versus $9.6 \mathrm{mg}$ ). A higher proportion of cognitively intact patients described pain at rest as 'none' or 'mild' $(89.47 \%$ versus $66.67 \%$; $\mathrm{OR}=4.25 ; \quad 95 \%$ CI: $0.64-28.25 ; p=0.134)$. However, some patients still described pain on movement as 'moderate' or 'severe'. This was significantly more frequent within cognitively intact patients $(79.0 \%$ versus $41.7 \%$; $\mathrm{OR}=0.19$; 95\% CI: $0.04-0.94 ; p=0.04)$ than for those with cognitive impairment.
Discussion: Despite our introduction of proactive individualised pain management within this orthogeriatric ward, cognitively impaired patients continue to receive lower doses of opioid analgesia and report persistent pain at rest.

Conclusion: There is no simple regime that will meet the needs of cognitively impaired patients, and the balance between the benefits and sideeffects of opioid analgesia must be central to every ward round if these people are to receive appropriate pain relief and so avoid the catastrophic consequences of delirium.

\section{Abstract ID: 44 \\ Clinical audit/service evaluation Subcategory: DXA}

Bone densitometry worldwide: a global survey by the ISCD and IOF

MA Clynes', LD Westbury', EM Dennison 1,2, JA Kanis $^{3,4}$, MK Javaid ${ }^{5}$, NC Harvey 1,6, M Fujita', C Cooper ${ }^{1,6,8}$, WD Leslie9, CR Shuhart ${ }^{10}$

'MRC Lifecourse Epidemiology Unit, University of Southampton, Southampton, UK, 2Victoria University of Wellington, Wellington, New Zealand, ${ }^{3}$ Mary McKillop Health Institute, Australian Catholic University, Melbourne, Australia, ${ }^{4}$ Centre of Metabolic Bone Diseases, University of Sheffield Medical School, Sheffield, UK, ${ }^{5}$ Nuffield Department of Orthopaedics, Rheumatology and Musculoskeletal Sciences, University of Oxford, Oxford, UK, ${ }^{6}$ NIHR Southampton Biomedical Research Centre University of Southampton and University Hospital Southampton NHS Foundation Trust, Southampton, UK, ${ }^{7}$ International Osteoporosis Foundation, Nyon, Switzerland, ${ }^{8}$ NIHR Oxford Biomedical Research Centre, University of Oxford, Oxford, UK, ${ }^{9}$ Department of Internal Medicine, Rady Faculty of Health Sciences, University of Manitoba, Winnipeg, Manitoba, Canada, ${ }^{10}$ Swedish Medical Group, Swedish Bone Health and Osteoporosis Center, Seattle, WA, USA

Background: Whilst the use of dual-energy x-ray absorptiometry (DXA) has been widely adopted worldwide for the assessment of bone mineral density (BMD), the quality of DXA facilities is unknown.

Objectives: To report the findings of a survey of Fracture Liaison Services (FLS) conducted by the International Society for Clinical Densitometry (ISCD) and the International Osteoporosis Foundation (IOF) assessing the quality of their DXA facilities.

Materials and Methods: A questionnaire for the accessibility and quality of DXA services was cocreated by representatives of the ISCD (International Society of Clinical Densitometry) and the IOF and made available to institutions who participated in Capture the Fracture (CTF) 
Best Practice Framework (BPF). From a list of 331 contacted invitees, 124 FLS centres responded; analyses were based on 121 centres with suitable data.

Results: Over $70 \%$ of institutions reported that, for over $90 \%$ of the time, DXA access met service needs and the scanning/reporting quality was perceived as excellent. However, $25 \%$ of DXA facilities reported not being accredited by a professional/governmental organisation and adherence to some basic DXA quality assurance and reporting procedures was confirmed by $<50 \%$ of services. Importantly, in excess of $50 \%$ of institutions stated that they desired ongoing education in osteoporosis and DXA for operators and interpreters.

Discussion: There is significant variability in the access to and quality of DXA services for established FLS worldwide.

Conclusion: Despite two decades of training initiatives in osteoporosis densitometry, many centres are falling short of the standards of the IOF-ISCD Osteoporosis Essentials criteria.

\section{Abstract ID: 46 \\ Clinical audit/service evaluation Subcategory: COVID}

\section{Virtual fracture liaison service clinics in the COVID era}

S English, L Coyle, S Bradley, W Wilton, J Cordner, R Dempster, JR Lindsay

Fracture Liaison \& Osteoporosis Service, Musgrave Park Hospital, Belfast, UK

Objective: We examined the impact of provision of virtual FLS telephone clinics, as an alternative to face to face clinics during the lockdown.

Methods: patients presenting with low trauma fracture were recruited according to standard criteria. A structured telephone clinic appointment was offered to those aged $\geqslant 50-80$ years, which included fracture risk $\&$ health promotion assessment and a treatment plan. We analysed outcomes from the first 100 patients who 'attended' virtual clinics. Risk factors, demographics, fracture type, FRAX scores and outcomes were analysed. We also assessed patient experience with an anonymised patient survey, with a rating scale of
1-5 (from poor-excellent) to describe their experience of the virtual FLS service.

Results: We observed high 'attendance' rates at $79 \%$, however a significant number were not available for telephone review (11\%) or cancelled their appointment (10\%). 77 women and 23 men were assessed (mean age 77.4 years). The mean 10 year observed fracture risk for major osteoporotic fracture was $25 \%$ and $8 \%$ for hip fracture. Smoking status (30\%), parental hip fracture (16\%) and secondary causes (16\%) were reported most frequently. A recommendation for bisphosphonate was identified in $54 \%$ of the cohort based on NOGG criteria. Treatment with calcium and/ or vitamin $\mathrm{D}$ was recommended in a majority of cases $(90 \%)$ alongside health promotion recommendations for falls prevention (97\%) and lifestyle management (98\%). Follow up DXA assessment is planned for $64 \%$, as part of the NOGG recommendation and/or general clinical care. We received 60 responses from the initial patient survey. $90 \%$ rated their overall experience of service at 4 or 5 (very good to excellent). $98 \%$ indicated they would recommend the service to others. 33/46 rated the ease of making an appointment by phone as excellent, 3 as very good, 6 as good and the remainder did not comment.

Discussion: Virtual FLS clinics are effective in delivery of fracture risk assessment, health promotion and clinical management and are well received by patients with positive patient experience. While a significant proportion will require DXA follow up to complete the clinical assessment virtual clinics may mitigate delays in fracture prevention interventions during COVID.

\section{Abstract ID: 48 \\ Clinical audit/service evaluation Subcategory: FLS}

\section{Using QI to improve FLS monitoring: how sustainable are the results}

PC Stevens 1, G White1, V Haeri', MK Javaid'1,2, S Connacher ${ }^{1}$, R Eckert ${ }^{1}$, P Osborne1, J Kelly', S Mahoney', C Milan', D Prieto Alhambra1,2, $R$ Low $^{1}$

${ }^{1}$ Fracture Prevention Service, Oxford University Hospitals Foundation Trust, Oxford, UK, ${ }^{2 N D O R M s, ~ O x f o r d ~ U n i v e r s i t y, ~ O x f o r d, ~ U K ~}$

Background: Patients need to adhere to antiosteoporosis medication to benefit. The current 
approach to improve adherence for FLSs is by monitoring patients 16 and 52 weeks after a sentinel fracture.

Objective: To increase our monitoring indicator at four months from $38 \%$ to $50 \%$, by July 2019 , and then increase to $75 \%$ by December 2019 as demonstrated by the FLS Database.

Method: Our FLS enrolled in the national RCPQI FLS Breakthrough Collaborative, which included three sessions with pre-meeting tasks and mentoring calls between sessions. We form a quality improvement team to develop a driver diagram describing the challenges and series of interventions. The impact of our interventions was measured using the FLSDB run charts as well as feedback from patients and nurses.

Results: We invited a patient to join our team, and this was key as it helped to change the driver diagram confirming there were multiple obstacles: patient compliance, staff knowledge, and IT issues.

Our primary intervention was switching from posting paper questionnaire to telephone follow up. Other interventions included a new pathway for patients in care homes, an educational day to update staff, modifying the patient letters and improving the quality of uploads to FLSDB. The impact was a four-month monitoring rate of $56 \%$ by July and $63 \%$ by December 2019 . Patients favoured whichever method they had been contacted by. The nurses reported the telephone follow up calls were more efficient and produced an improved patient outcome, as patients were able to have any concerns or issues addressed immediately. We estimated switching to telephone monitoring saved $£ 8000$ per year.

Discussion: The RCPQI project gave 'permission to spend time' on improving our service.

Although we thought the telephone monitoring was the only intervention, the QI process highlighted several issues that had to be addressed.

Conclusion: Even though we had participated in the FLSDB since 2016, taking part in RCPQI collaborative has delivered improvements in patient outcomes. While we achieved our shortterm objective, further QI is needed to take our monitoring indicator to $80 \%$.

\section{Abstract ID: 50 \\ Clinical audit/service evaluation Subcategory: Vertebral fracture}

\section{Prevalent vertebral fracture in patients with acute hip fracture}

JGL Griffin ${ }^{1,2}$

'University Hospitals Plymouth NHS Trust, Plymouth, UK, ${ }^{2}$ Royal Osteoporosis Society, Bath, UK

Background: Mortality in osteoporotic hip fracture is significant with $6.1 \%$ of patients dying within 30 days of fracture and $1 / 3$ within 12 months $s^{1,2}$. Fracture liaison services identify fragility fractures and intervene with appropriate assessment and treatment for the further prevention of fragility fractures. ${ }^{3,4}$ Only approximately $6 \%$ of vertebral fragility fractures are identified by FLS through routine case finding ${ }^{5}$ however many diagnostic imaging tests include the spine which provides a valuable opportunity to identify patients a risk of fracture.

Methods: The imaging history of 176 patients with hip fracture was investigated and any crosssectional imaging reports in the 5 years prior to hip fracture scrutinised to identify the report of vertebral fracture. Indications for the investigation were noted as unrelated to bone health, trauma or vertebral fracture.

Results: $74(42 \%)$ of the cohort had cross sectional imaging in the 5 years prior to hip fracture. $22 \%$ of the reports included vertebral fracture or terminology suggestive of fracture for example, wedge, end plate depression.

Discussion: $22 \%$ of hip fracture patients in this study had a prior fragility fracture and a missed opportunity for secondary fracture prevention. The prevalence is similar to that reported in the general population of those aged over 50 years ${ }^{6,7}$. This study did not scrutinise the imaging to verify report finding or identify vertebral fractures that were unreported and therefore results and prevalence may be underestimated.

\section{References}

1. Royal College of Physicians. National hip fracture database annual report. London: Royal College of Physicians, 2019.

2. Public Health England. NHS right care: falls and fragility fractures. London: Public Health England, 2017. 
3. National Institute for Clinical Excellence. Osteoporosis quality standard QS149. London: National Institute for Clinical Excellence, 2017.

4. Royal Osteoporosis Society. Effective secondary fracture prevention of fragility fractures: clinical standards for fracture liaison services. Bath: Royal Osteoporosis Society, 2019.

5. Royal College of Physicians. Fracture Liaison service database commissioners report. Royal College of Physicians, 2019.

6. O'Neill W, Felsenberg D, Varlow J, et al. The prevalence of vertebral deformity in European men and women: the European Vertebral Osteoporosis Study. F Bone Mineral Res 1996; 11: 1010-1018.

7. Cooper C, Atkinson EJ, O'Fallon WM, et al. Incidence of clinically diagnosed vertebral fractures. F Bone Mineral Res 1992; 7: 221-227.

\section{Abstract ID: 51 \\ Clinical audit/service evaluation Subcategory: DXA}

\section{Are DXA scans in the older adult clinically important?}

\author{
J Knell' ${ }^{1}, J G L$ Griffin ${ }^{1,2}$ \\ 'University Hospitals Plymouth NHS Trust, Plymouth, UK, ${ }^{2}$ Royal \\ Osteoporosis Society, Bath, UK
}

Background: Bone mineral density measurement (BMD) by dual energy x-ray absorptiometry (DXA) is the preferred diagnostic method for the diagnosis of osteoporosis in adults. ${ }^{1,2}$ NICE guidance on treatment of osteoporosis for primary and secondary prevention of fragility fractures ${ }^{3,4}$ suggest that 'a diagnosis of osteoporosis may be assumed in females over the age of 75 years or older if the responsible clinician considers a DXA scan to be clinically inappropriate or unfeasible.' This has led to barriers for access to DXA for this age group.

Method: This audit evaluated 100 DXA referrals and the acceptance rate of those for people aged 75 and older, and the clinical outcomes of fracture risk assessment with and without BMD measurement.

Results: $19 \%$ of referrals were for patients aged 75 or over. $84 \%$ of these were accepted for scanning. The average fracture risk $\left(\mathrm{FRAX}^{\circledR}\right)^{5} 10$ year probability of a major osteoporotic fracture: excluding BMD measurement was $22.32 \%$ and including BMD measurement was $18.2 \%$.
Discussion: The fracture risk was significantly influenced by the inclusion of BMD in $47 \%$ of cases.

Conclusion: The majority of patients aged 75 and over referred for DXA are accepted and DXA is a useful tool in the around half of these patients to refine fracture risk and make treatment decisions including the decision not to treat. Identifying those where this benefit may be perceived was not predictable prior to scanning.

\section{References}

1. World Health Organisation. Assessment of fracture risk and its application to screening for postmenopausal osteoporosis: report of a WHO study group [meeting held in Rome from 22 to 25 Fune 1992]. WHO Technical Report Series 843, 1994. Geneva: WHO.

2. International Society for Clinical Densitometry. (2019) Official Positions-Adults. https://www.iscd .org/official-positions/2019-iscd-official-positionsadult/ accessed May 2020

3. National Institute for Clinical Excellence. Raloxifene for the primary prevention of osteoporotic fragility fractures in post-menopausal women: technology appraisal guidance [TA160]. London: NICE, 2018.

4. National Institute for Clinical Excellence. Raloxifene and teriparetide for the secondary prevention of osteoporotic fragility fractures in post menopausal women: Technology appraisal guidance [TA161]. London: NICE, 2018.

5. University of Sheffield (2008), https://www .sheffield.ac.uk/FRAX/ accessed May 2020

\section{Abstract ID: 52 \\ Clinical audit/service evaluation Subcategory: COVID}

\section{"Covid-19 learning: a telephone triage system for treatment/discharge in a DXA scanning service}

\author{
J Knell' ${ }^{1}$, JGL Griffin ${ }^{1,2}$ \\ 'University Hospitals Plymouth NHS Trust, Plymouth, UK, ${ }^{2}$ Royal \\ Osteoporosis Society, Bath, UK
}

Background: The global COVID-19 pandemic resulted in a 'lockdown' and restrictions of movement within the UK. As a result, many bone densitometry (DXA) services suspended scanning. The risk of bringing patients out of their homes for their DXA scan was assessed and noted to be greater than the risk of delaying patient DXA scans in the management of fracture risk and osteoporosis. In 
order to support patients access to treatment where they were of high fracture risk a telephone triage system was developed and rolled out.

Methods: A telephone triage system was set up for patients to be contacted for a consultation with a specialist DXA radiographer. During this appointment questions were asked specific bone health and lifestyle questions that correlated to the national Fracture Risk Assessment Tool (FRAX). ${ }^{1}$ The individuals responses were input into the FRAX tool online and the fracture risk was calculated and $\mathrm{NOGG}^{2}$ guidance viewed. The outcome then prompted what advice would be offered to the referrer and patient in accordance with local and national protocols and guidance.

Results: Over a ten week period 270 patients undertook telephone consultations, $61(23 \%)$ patients were given lifestyle advice, reassurance and discharged to their primary care practitioner, 152 (56\%) patients were recommended to be scanned (and placed onto a planned waiting list) 57 (21\%) of patients were recommended treatment as per $\mathrm{NICE}^{3}$ guidelines and the referrer was asked to re-refer for a quasi-baseline DXA following lockdown.

Conclusion: A telephone triage system has been able to support high risk patients access treatment without a DXA scan supporting secondary fracture prevention. Patients understood why their scan was delayed and appreciated information to support them looking after their bone health in the mid-term. Discharging patients at low risk will support onward waiting list management following easing of lockdown restrictions in the UK. Pathways developed have supported the development of a Royal Osteoporosis Society DXA (COVID19) restoration toolkit. ${ }^{4}$ Trailing a telephone triage may support development of new pathways for those over 75 in a post COVID-19 era.

\section{References}

1. University of Sheffield (2008), https://www .sheffield.ac.uk/FRAX/ accessed March 2020

2. National Osteoporosis Guidance Group (2017) https://www.sheffield.ac.uk/NOGG/index.html Accessed March 2020

3. NICE (National Institute for Health and Care Excellence) https://www.nice.org.uk/guidance/ ta464 Accessed March 2020

4. The Royal Osteoporosis Society, (2020) Toolkit: DXA restoration of service https://theros.org.uk/ healthcare-professionals/covid-19-hub/ Accessed June 2020

\section{Abstract ID: 53 \\ Clinical audit/service evaluation Subcategory: Management and treatment}

\section{Audit on denosumab prescribing in the elderly in a large teaching hospital}

\section{Sayed, R Dwivedi}

Health Care for Older People, Nottingham University Hospitals NHS trust, Nottingham, UK

Background: Denosumab is approved for the treatment of osteoporosis in postmenopausal women and men at increased risk of fractures (Cummings et al. 2009, Langdahl et al. 2015). It remains the only treatment option in patients with advanced kidney disease (Block et al. 2020).

Objectives: We set out to assess our current departmental practice of Denosumab prescribing in accordance with the NOGG (National Osteoporosis Guideline Group) 2017 guidance. Our aim was to standardise our management and monitoring protocol for patients on Denosumab and to come up with trust wide recommendations.

Methods: A retrospective audit on 50 patients who have received Denosumab across five different osteoporosis clinics between January 2015 and March 2020 was carried out. Data was captured on indication, bone biochemistry monitoring, documentation of consent, monitoring for rare side effects etc. Data was obtained from review of clinic correspondence and clinical information obtained from hospital IT systems.

Results: Majority of the patients were females (46/50) with a mean age of 82.5 years. CKD (Chronic Kidney Disease) was the major indication of Denosumab (29/50) followed by intolerance or contraindication to bisphosphonate (12/50), and ineffective response to bisphosphonates (11/50). Among patients with CKD, bone biochemistry monitoring and renal follow up was in place for only seven out of $18(39 \%)$ patients with Creatinine Clearance $<30$. Only four patients were enquired about dental issues $(8 \%)$ and only 11 patients (22\%) were provided information leaflets about this injection.

Discussion: Bone biochemistry monitoring is required for all patients with advanced CKD due to the risk of hypocalcemia. Similarly, information leaflets needs to be offered to all patients or their families prior to initiating treatment and there is a need to improve documentation of 
consent and monitoring for rare side effects in our clinic correspondence.

Conclusion: The findings of this audit has been shared with our colleagues to improve practice and will form the basis of a future business case for joint care with the renal team of patients with advanced CKD. We plan to re-audit in 12 months to assess improvement.

\section{Abstract ID: 56 \\ Clinical audit/service evaluation \\ Subcategory: Vertebral fracture}

\section{NICE guidance 41: is it applicable to a real- world cohort of cervical spine fractures in older people?}

FHA Gilbert' ${ }^{1}$ D Mony' ${ }^{1}$ N Fuller², O Stokes², M Brown ${ }^{2}, K_{\text {Knapp }}^{3}$, EM Clark ${ }^{1,4}$

${ }^{1} T$ ranslational Health Science, University of Bristol, Bristol, UK, ${ }^{2 T h e}$ Royal Devon and Exeter Hospital, Exeter, UK, ${ }^{3}$ University of Exeter, Exeter. UK, ${ }^{4}$ Rheumatology Department, North Bristol NHS Trust, Bristol, UK

Background: Currently cervical spine fractures (CSFracs) in the elderly resulting from a low trauma mechanism of injury are under researched. Clinically there are missed opportunities around delays in diagnosis due to inappropriate use of imaging, and lack of consideration for future fracture prevention. NICE Guidance 41 covers the assessment and initial management of CSFracs, using the Canadian C-spine rule to identify if people are at risk of a CSFrac.

Objective: To use a hospital cohort of CSFracs in older adults collected over a four-year period to identify if the rules highlighted in NG41 are appropriate.

Methods: Data were extracted from the medical notes from all adults aged $>50$ diagnosed with a CSFrac at the Royal Devon and Exeter Hospital over a 4-year period, as part of a service evaluation. Data collected included mechanism of injury plus self-reported symptoms on presentation to A\&E. As part of a Year three medical student choice project, these data were analysed to identify if the injury mechanism and symptoms in this real-world cohort were similar to that described in the Canadian C-spine rule highlighted in NG41.

Results: 84 older adults were identified with at least one CSFrac during this 4-year period. Only $32.1 \%$ described a 'dangerous mechanism of injury' according to the description in NG41. Conversely, $69.0 \%$ of those aged $>65$ described a ground level fall. In addition, only $6.0 \%$ described paraesthesia in the upper or lower limbs. Of those aged $>65,25.4 \%$ would be classified as high risk of CSFrac according to NG41. The majority of the cohort described neck pain, but this does not feature in the Canadian C-spine rule.

Discussion: Relying on the Canadian C-spine rule only, as recommended by NG41, may be contributing to delay in diagnosis of CSFrac in three-quarters of older adults, as age $>65$ was the biggest predictor. We recommend adding ground level falls and neck pain into the guidance. This modified guidance needs testing in a real-world setting.

\section{Abstract ID: 57 \\ Clinical audit/service evaluation Subcategory: Vertebral fracture}

\section{${ }^{*}$ Prevalence of vertebral fragility fractures in patients aged $\mathbf{7 0}$ years and older attending computed tomography imaging in UK radiology settings}

\section{C Howlett ${ }^{1}$, K Drinkwater $^{2}$, N Mahmood $^{1}$,} J Illes ${ }^{3}$, J G L Griffin ${ }^{4}$, K Javaid ${ }^{5}$

${ }^{1}$ Radiology, Eastbourne Hospital, Eastbourne, UK, ${ }^{2}$ The Royal College of Radiologists, London, UK, ${ }^{3}$ Radiology, Dorset County Hospital, Dorchester UK, ${ }^{4}$ The Royal Osteoporosis Society, Bath, UK, ${ }^{5}$ The Nuffield Department of Orthopaedics, Rheumatology and Musculoskeletal Sciences, Oxford, UK

Background: Osteoporotic vertebral fractures (VFx) are the commonest fragility fracture presenting in $12-20 \%$ of women aged $50+$ according to data published $>10$ years ago. Outcomes of VFx include increased morbidity and mortality and yet up to $70 \%$ of Vfx do not come to medical attention/remain undiagnosed. The presence of a Vfx is also a powerful predictor of future fracture presenting a critical opportunity to intervene with secondary fracture prevention measures. Opportunities are missed during imaging studies to report on Vfx that are incidental to the primary indication for the imaging test.

Method: This study was designed to investigate the presence of vertebral fragility fracture in patients aged 70 and over attending UK imaging centres for computed tomography (CT) scans for indications unrelated to Musculo-skeletal conditions. National radiology audit leads undertook retrospective data gathering at their local centre 
by scrutiny of 50 consecutive CT scans that included the thoracolumbar spine in patients age 70 or over. Trauma, known bony metastatic disease and myeloma were excluded. Vfx identification criteria used was the Genant semi-quantitative method, and Grade 1 (mild) fractures were not included in order to exclude confounders that may mimic grade 1 fractures.

Results: 6357 patients' scans were evaluated at 127 centres. $1362(21.4 \%)$ scans demonstrated one or more grade $2 / 3 \mathrm{Vfx}$.

Discussion: Prevalence is concordant with data published by studies $>10$ years ago. Reporting and onward referral for secondary fracture prevention were also investigated and found that $5.5 \%$ of the reports identifying Vfx recommended further investigation for osteoporosis and only $0.8 \%$ of the reports were regarded by auditors as 'actionable' by the referrer. The number of vertebral fractures identified in this study was not reported.

Conclusion: Prevalence of vertebral fragility fracture has remained static in the past decade suggesting that prevention of vertebral fragility fracture strategies have yet to be fully defined or effective and incidence has not reduced. CT imaging that includes the spine is a valuable opportunity to intervene where there are previously unreported vertebral fracture which can prevent further fracture, morbidity and mortality. Current systems are not effective in identifying and acting on incidentally found vertebral fracture

\section{Abstract ID: 60 \\ Clinical audit/service evaluation Subcategory: Vertebral fracture}

\section{Improving vertebral fracture identification by the fracture liaison service}

\footnotetext{
J McGregor ${ }^{1}$, H Watts ${ }^{2}$, S Tuck ${ }^{3}$, S Pletts ${ }^{1}$

${ }^{1}$ Osteoporosis Service, South Tees NHS Foundation Trust, Middlesbrough, UK, ${ }^{2}$ Radiology Department, South Tees NHS Foundation Trust, Middlesbrough, UK, ${ }^{3}$ Rheumatology Department, South Tees NHS Foundation Trust, Middlesbrough, UK
}

Background: The Fracture Liaison service aims to identify low trauma fractures for patients within a large population CCG area. The service had no robust pathway in place to identify patients sustaining vertebral fractures as many of these are found opportunistically and do not attend Accident and Emergency. This is missing opportunities to assess patients. The Service identified 48 vertebral fractures in 2018 which is $11 \%$ of estimated cases for estimated population size of 273,000. National average of identification of vertebral fractures was $36 \%$

Objective: Improve the identification of vertebral fracture by the local fracture liaison service

Methods: Initially Osteoporosis Specialist Nurse reviewed if identification of fractures was possible using search terms built in to the IMPAX system. Further meetings were then planned with stakeholders in Radiology department leading to a report published monthly to the service of all lumbar and thoracic images with term "vertebral fracture" in report. The Advanced Practitioner Reporting Radiographer agreed with the service to implement a search code in reports to directly identify patients to the service with new /or not previously identified vertebral fractures.

Results: Following the collaborative working with radiology system teams and Advanced Practitioner Reporting Radiographer, 138 patients who had sustained a vertebral fracture where identified by the service in 2019 compared to 48 in 2018. Figures for 2020 up to the 25 th June indicate a continued improvement in identification, with 93 patients with vertebral fracture identified so far.

Discussion: The initial report provided the Radiology Systems Team increased the identification significantly, however was unreliable with identification. This proved time consuming for the Fracture Liaison Service, on average 179 possible patients were identified by this report when only an average of 31 patients had vertebral fracture. A new Standard Operating Procedure has been agreed by reporters in the trust to place a short code in the report for appropriate patients.

Conclusion: The collaborative work between the Fracture Liaison Service, Radiology Systems Team and The Advanced Practitioner Reporting Radiographer successfully improved the identification rate of vertebral fractures. Further review of the effectiveness of the short code included in the report will be required. 


\section{Abstract ID: 68 \\ Clinical audit/service evaluation Subcategory: Vit D}

\section{A specialist pharmacist clinic in hard-to- treat vitamin D deficiency}

\author{
LA Statham ${ }^{1,2}, V$ Woodhall2, F Cooles², \\ TJ Aspray ${ }^{2}$ \\ 'School of Pharmacy, University of Sunderland, Sunderland, UK, ${ }^{2 T h e}$ \\ Bone Clinic, Freeman Hospital, Newcastle upon Tyne Hospitals NHS
} Foundation Trust, Newcastle, UK

Background: Our metabolic bone clinic treats patients with persistent vitamin D deficiency, many of whom also need treatment for osteoporosis. In 2018, while 800 patients received parenteral antiresorptive therapies, $13 \%$ had to defer because their serum $25(\mathrm{OH})$ vitamin $\mathrm{D}(25 \mathrm{OHD})$ was low.

Objective: To review the impact of a specialist vitamin $\mathrm{D}$ clinic on our service.

Methods: Patients with low 25OHD attended our Pharmacist-led clinic for review and consideration of buccal vitamin D therapy, which is a "red" hospital-only drug locally. Previous prescriptions were reviewed, therapy amended and 25OHD levels were monitored. A hospital prescribing audit was performed for patients prescribed buccal colecalciferol, and demographic and clinical details were collated from hospital records.

Results: 24 patients were identified. 17 were from our service: $12: 5$ were female:male with a mean (range) age of 56 (34-82) years. All had osteoporosis and low 250HD [mean (range) $26(<13$ $41 \mathrm{nmol} / \mathrm{L})]$. Reasons for referral comprised: malabsorption due to Crohn's disease/short bowel (9), pancreatic insufficiency (2), other (2) or intolerance to oral preparations (4). Serum 250HD and calcium were checked at 1 month, then 2-3 monthly, with dose titration, until a stable $25 \mathrm{OHD}$ was maintained. Mean (range) starting dose and final dose of colecalciferol were 3000 (1000-9000) IU and 6000 (3000-9000IU) daily; and it took 9 (3-16) months to achieve threshold 25OHD $(50 \mathrm{nmol} / \mathrm{L})$ in 8 of 12 patients. Reasons for four patients stopping were: sore mouth (1), prescriptions not collected (2), low 25OHD despite 9000 IU/d (1). 10 of 12 patients successfully received parenteral treatment: ibandronic/zoledronic acid (5), denosumab (5), with two patients declining.

Discussion: Two-thirds of people, who failed using standard vitamin D loading regimens, responded. Barriers to effectiveness included: poor adherence and the need to attend hospital for therapy. Although it took an average of 9 months to reach target, more aggressive dosing might allow antiresorptive medication to be given sooner. The system required a pharmacist prescriber and regular patient engagement.

Conclusion: Buccal vitamin D enabled antiresorptive therapies to start in $>80 \%$ of patients with persistently low vitamin D levels, and optimised use of day case appointments.

\section{Abstract ID: 69 \\ Clinical audit/service evaluation \\ Subcategory: Hip fracture}

\section{Review of the care home divide: secondary fracture prevention before admission with a hip fracture}

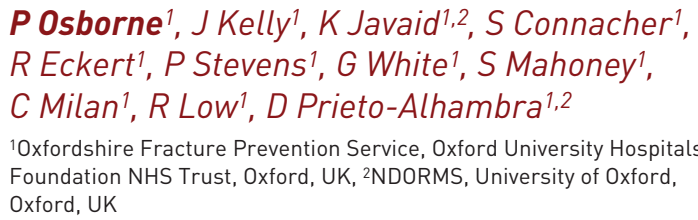

Introduction: Secondary fracture prevention has been demonstrated to be clinically and costeffective. However, the number of patients with a prior fracture who are treatment naïve remains high despite the previous fracture being an important predictor of future fracture. In 2018 we demonstrated 55\% care home patients admitted with a hip fracture and with a prior fracture had received secondary fracture prevention. To improve this, adapted communication between our FLS and care homes in terms of recommendations, monitoring and working with the local care home support team. This audit re-visits data in 2018 to review if the practice has improved further for care home patients and those still living in their own home.

Materials and Method: Patients who had sustained a hip fracture in the local area were identified from the local FLS database. We identified those patients admitted from a care home and also if they had previously fractured on treatment using NHS records and patient self-report. We classified a care home as a Residential or Nursing Home. Treatment was classified as an ongoing prescription of any bone protection medication at the time of the hip fracture. 
Results: From a sample of 50 patients admitted from a care home in 2019 with a hip fracture, $40 \%$ had a previously reported fracture, and $55 \%$ were prescribed bone protection treatment. In comparison patients with a hip fracture who were admitted from their own home, $24 \%$ had a previous fracture, and $17 \%$ were prescribed treatment. In 2018 , patients admitted from a care home $36 \%$ had a previous fracture, and of these $55 \%$ were on treatment. In comparison patients admitted from their own home, $35 \%$ had a previous fracture $38 \%$ were on treatment.

Conclusion: The number of patients already on secondary fracture who are admitted from care homes has NOT increased since the implementation of a Fracture Prevention Service and further improvement work is needed.

\section{Abstract ID: 73 \\ Clinical audit/service evaluation Subcategory: Management and treatment}

\section{An audit cycle of renal bone disease against kdigo guidelines}

N Cernovschi-Feasey', S Zeb' ${ }^{1}$, T Salter $^{2}$, $M$ Lloyd $^{1}$

${ }^{1}$ Rheumatology, Frimley Park Hospital, Frimley, UK, ${ }^{2}$ Nephrology, Frimley Park Hospital, Frimley, UK

Background: Chronic kidney disease-mineral and bone disorder (CKD-MBD) is complex and management can be difficult. We aimed to compare management of our CKD patients to 2018 KDIGO guidelines. The guidelines suggest checking calcium and phosphate $\left(\mathrm{Ca}^{2}+/ \mathrm{PO}_{4}\right)$ within 12 mths in CKD $3 a$ and $3 b$, within 6 months for CKD 4, and within 3 months for CKD 5. Parathyroid hormone (PTH) should be checked at baseline in CKD 3a-b, within 12 months in CKD 4 and within 6 months in CKD 5. Alkaline phosphatase (ALP) should be measured within 12 months in CKD 4 and 5. 25-(OH)D levels 'might' be measured at baseline in CKD 3a to 5D. BMD scanning is suggested if the result will impact treatment decisions. Calcitriol and vitamin $\mathrm{D}$ analogues are no longer routinely advised in CKD 3a-5; 25-(OH)D insufficiency should be corrected as in the normal population.

Methods: We randomly selected 70 patients to audit from renal clinics in 2019, and repeated the audit 6 months later in 2020, randomly selecting 70 different patients.
Results: Audit 1: Mean age 67, 41 M, 29 F. 47\% CKD 3a-b; 44\% CKD 4; 9\% CKD5. Audit 2: Mean age $67,42 \mathrm{M}, 28 \mathrm{~F}, 37 \%$ CKD 3a-b; $56 \%$ CKD $456 \% ; 7 \%$ CKD 5 . The results of testing and treatment against guidelines are presented below:

\begin{tabular}{|l|l|l|}
\hline \multicolumn{3}{|c|}{ Tests / treatment as per guideline (\% of patients) } \\
\hline & Audit 1 & Audit 2 \\
\hline $\mathrm{Ca}^{2+} / \mathrm{PO}_{4}$ & 97 & 100 \\
\hline $\mathrm{ALP}$ & 100 & 100 \\
\hline $\mathrm{PTH}$ & 36 & 25 \\
\hline $25-(\mathrm{OH}) \mathrm{D}$ & 24 & 40 \\
\hline DXA & 17 & 8 \\
\hline Bone biopsy & 0 & 0 \\
\hline Activated vitamin D Rx & 14 & 0 \\
\hline 25-(OH)D analogue $\mathrm{Rx}$ & 18 & 21 \\
\hline Bisphosphonate Rx & 4 & 0 \\
\hline
\end{tabular}

Conclusions: The two groups were well matched for age; Audit two had relatively more CKD four patients. PTH testing remained relatively low but we saw improvement in vitamin $\mathrm{D}$ testing and treatment. Bone biopsy was not performed in either group.

\section{Abstract ID: 75 \\ Clinical audit/service evaluation Subcategory: Fracture risk}

Optimising fracture risk assessment in patients with prostate cancer on androgen deprivation therapy: FRAX and more?

N Hannaway' ${ }^{1}$ X Jiang ${ }^{1}, J$ Ferguson'2, A Burns', T Watson', I Pedley', J Frew', A Azzabi', $R$ Pearson', R Chandler', R McMenemin', A Bennett ${ }^{1}$, H Showler ${ }^{1}$, T Aspray ${ }^{4}$

${ }^{1}$ Oncology, Northern Centre for Cancer Care, Newcastle upon Tyne, UK, 2 Urology, Freeman Hospital, Newcastle upon Tyne, UK, ${ }^{3}$ Nuclear Medicine, Freeman Hospital, Newcastle upon Tyne, UK, ^Rheumatology, Freeman Hospital, Newcastle upon Tyne, UK

Background: Patients with prostate cancer are at increased risk of adverse skeletal events including bony metastasis and treatment induced osteoporosis. FRAX is commonly used to estimate osteoporotic fracture risk but evidence for its use in patients with prostate cancer is limited.

Objective: (1) Estimate fracture risk in prostate cancer patients at diagnosis. (2) Inform the development for bone health management in this patient group. 
Methods: Between 2016 and 2018, 111 consecutive patients commencing ADT therapy at a regional cancer centre were seen and data collected prospectively on patient demographics, androgen deprivation therapy (ADT) duration and additional anticancer treatments. Patients underwent a baseline DXA scan and FRAX score was calculated. Unfortunately, repeat DXA at 2 years is delayed due to COVID-19.

Results: Median age was 73 years. 39 (35\%) patients had bone metastases, of whom 16 had additional lymph node metastases. Mean 10-year fracture risk for patients without metastases/with metastases was $9.6 \% / 9.9 \%$ for major osteoporotic and $4.2 \% / 4.6 \%$ for hip fracture. 91 patients had DXA at baseline with mean T-score without/with metastases of $0.7 / 0.4$ (spine), $-0.8 /-1.1$ (femoral neck) and 0.1/0.4 (total hip).

Discussion: Most prostate cancer patients now receive multimodal anticancer treatment. Average overall survival for patients presenting with metastatic disease is now greater than 5 years. Long term ADT use increases osteoporotic fracture risk and pelvic radiotherapy is associated with sacral insufficiency fracture, while additional anticancer treatments can cause accelerated bone loss. FRAX was developed in other clinical contexts than prostate cancer and may significantly underestimate the fracture risk in these patients.

Conclusion: The true value of FRAX and DXA are unknown in prostate cancer patients. Given the increased risk of bone events, fracture risk should be calculated at baseline as a standard for these patients and surveillance performed in patients with increased risk. A lower threshold for commencement of bone protective agents may be appropriate at baseline given anticipated negative effects on bone density and a high incidence of skeletal events.

\section{Abstract ID: 76 \\ Clinical audit/service evaluation Subcategory: Fracture risk}

\section{UK FRAX Vs. Indian FRAX in South Asians in UK}

S Arya, S Smitten, H Sapkota, S Venkatachalam

Cannock and Wolverhampton Rheumatology Centre, The Royal Wolverhampton NHS Trust, Wolverhampton, UK

Background: The FRAX tool (www.shef.ac.uk/ FRAX) calculates the 10-year probability of hip fracture or a major osteoporotic fracture based on clinical risk factors and bone density. It varies depending on ethnicity and the epidemiological data on osteoporotic fractures from different countries. We wanted to address any discrepancy in treatment recommendations following a DXA scan in South Asians applying UK FRAX.

Objectives: To compare performance of FRAX for UK population with FRAX for the Indian population in South Asians in UK.

Methods: Retrospective analysis of data from 100 consecutive South Asian patients who had a DXA Scan at the Cannock and Wolverhampton Rheumatology Centre between April and July 2019. All patients answered a FRAX questionnaire before the DXA scan and the original recommendations were based on the UK FRAX.

Results: The mean age of patients was $64.5 \pm 10.8$ years. There were 79 females and 21 males. The mean BMI of patients was $27.7 \pm 5.1 \mathrm{~kg} /$ $\mathrm{m}^{2}$. FRAX risk factors included previous fragility fracture in $45 \%$ of the patients, history of parental hip fracture in $12 \%$ had and rheumatoid arthritis in $9 \%$. The mean femoral neck BMD was $0.85 \pm 0.15 \mathrm{~g} / \mathrm{cm}^{2}$ and $\mathrm{T}$-score $-1.24 \pm 1.22$. The correlation coefficient between UK and Indian FRAX was 0.76 . UK FRAX recommended treatment in 34 patients, but Indian FRAX in only 23 patients. 11 more South Asian patients in UK were advised treatment based on UK FRAX.

Conclusion: Assessing fracture risk in South Asians by UK FRAX tool leads to more patients getting treated as compared to using Indian FRAX tool. Indian FRAX tool is preferred in patients of South Asian ethnicity in UK to avoid over treatment of osteoporosis.

\section{Abstract ID: 77 \\ Clinical audit/service evaluation Subcategory: COVID}

The audit of phone consultations for osteoporosis; a valuable service during COVID and beyond

\section{AL Dolan}

Rheumatology Dept, Queen Elizabeth Hospital (Lewisham and Greenwich Trust), Woolwich, London, UK

Recent debate has acknowledged the traditional model of outpatient care is no longer fit for 
purpose, as discussed in the Royal College of Physicians report, 'Outpatients: the future', 2018. This is particularly true for patients with Osteoporosis, who may be frail, elderly and for whom travel to clinic is difficult. Much of the consultation will depend on discussion and review of a bone density report rather than examination findings.

The uptake of virtual clinics had been slow, however all this changed in March 2020 with the Covid-19 epidemic. There was rapid change to deliver virtual consultations, which now needs analysis in order to consider their role in the future delivery of Osteoporosis services.

We report an audit of Phone Consultations undertaken in a Rheumatology Department during the Covid-19 epidemic.

Results: 140 Rheumatology telephone consultations took place in May 2020. 38 were for Osteoporosis including four with vertebral deformities on CT. 29 were new referrals, nine follow-up. Patients were pre-warned of the consults by text or phone call. 32 answered the call personally (two no response, one carer, three dementia/care home). 25 were deemed to be fully assessed by phone with appropriate management plan generated. In seven decision-making was limited by lack of DEXA. Zolendronate was offered to 12 , but due to Covid-19 service closures, could not proceed; six also required dental assessment. None were limited by inability to examine, in this sample. Eight were offered follow-up to complete discussion after DEXA or reading information.

Conclusions: Phone consultations provide an effective method of patient consultation for Osteoporosis, allowing discussion of treatment options, side-effects and plans for ongoing management. During Covid-19, closure of services delayed management, including DEXA availability, access to dental assessment and closed Zolendronate infusion service, which would normally not limit care. Lack of examination may miss some cases of vertebral fracture and there is a need to question on presence of spinal curvature or backpain and offer a face-to-face consultation and imaging if there is any suspicion. As a result of these findings phone consultations will continue to be offered as the initial point of contact for our Osteoporosis service.

\section{Abstract ID: 78 \\ Clinical audit/service evaluation Subcategory: DXA}

\section{Enabling patients: changes in \\ bisphosphonate prescription rates before and after service change in DXA}

K Gould ${ }^{1}$, T Malpas
1, M Tian-Clarke1,
F Crawford-Manning

1Osteoporosis Service, Midlands Partnership NHS Foundation Trust, Haywood Hospital, Stoke-on-Trent, UK, 2 School of Primary, Community and Social Care, Keele University, Staffordshire, UK

Background: Improving communication between primary and secondary care services may be important in addressing the osteoporosis treatment gap. Within our osteoporosis service at a communitybased hospital all DXA reports contain treatment recommendations. Previously, local procedure meant that the GP was the only one who received written treatment recommendations. With the aim of increasing the proportion of patients who actually initiated treatment, in January 2019, the service changed to send an additional patient letter notifying them of the need for treatment.

Objective: To compare prescription rates of bisphosphonates before and after the implementation of a patient letter indicating the need for medication.

Methods: A service evaluation was conducted. Osteoporosis service reports were reviewed for the first 3 months of 2018 (prior to the service change) and the first 3 months post change (Jan to June 2019) to identify all those in whom treatment was recommended. Using the Spine portal record of repeat prescriptions, for each patient who had received a treatment recommendation, we identified whether they were or not receiving a prescription in May 2020.

Results: $126 / 552$ (22.8\%) and 190/771 (24.6\%) patients received a treatment recommendation pre- and post-service change, respectively. Of those receiving a treatment recommendation $57 / 126(45 \%)$ and $104 / 190$ (55\%) were prescribed treatment, representing a $10 \%$ increase in prescription rates.

Discussion: One major limitation of this audit is that prescription was recorded 2 years after recommendation for the group with treatment recommendations pre-service change, and 1 year 
after recommendation for the post-service change group. Unfortunately, it was not possible to identify historic prescribing records. It is therefore possible prescribing rates are lower in the first cohort due to reducing persistence with bisphosphonates over time. However, these results suggest that our letter, designed to involve and enable patients may be an important component of facilitating smooth secondary-primary care communication and may have improved uptake of treatment. Given that $45 \%$ recommendations were still not followed, more needs to be done to facilitate uptake of secondary care treatment recommendations.

\section{Abstract ID: 84 \\ Clinical audit/service evaluation Subcategory: Management and treatment}

Bone health plan by orthogeriatricians: does it always get followed through?

\section{Berbecila, AR Lewis, L Marshall, NK Weerasuriya, MH Poon \\ Trauma and Orthopaedic, Nottingham University Hospitals, Nottingham, UK}

Background: Bone health assessments are performed on all hip fracture patients as part of the Best Practice Tariffs. Future fracture prevention is discussed with patients and a plan made for outpatient DEXA scan, antiresorptive treatment or both. Unfortunately, following discharge, some patients decide not to go ahead with the agreed plan.

Objective: To establish the number of patients who did not comply with their bone health plan as recommended by the orthogeriatricians, and to determine their subsequent management in the community and clinical outcomes.

Methods: This study was undertaken retrospectively on patients over age 60 with fragility hip or femoral fractures who were admitted to a large university hospital between January 2019 and April 2020, using three hospital databases.

Results: 835 patients were treated for acute hip or proximal femoral fractures and a bone health plan was agreed, however $11.3 \%$ did not follow through with this plan once discharged. Those who did not have a confirmatory DEXA were less likely to receive empirical treatment from primary care $(70 \%$ versus $30 \%)$, despite a written request to GPs to consider this as they had not received treatment in secondary care. Incidence of fracture, however, was not increased compared to those who did receive treatment $(14.3 \%$ versus $16.7 \%)$. Of those who turned down the offer of antiresorptive from secondary care, most remained untreated in primary care $(70.6 \%$ versus $30.4 \%)$. Again, there was no increase in number of fractures in the untreated group. 26 patients were offered both DEXA and antiresorptive therapy. $34.9 \%$ declined both, and none were subsequently treated empirically in primary care. $61.5 \%$ declined DEXA but agreed to having intravenous antiresorptive therapy, suggesting that patients are willing to accept treatment without confirmatory scan. $3.9 \%$ had a normal DEXA scan and therefore not treated.

Conclusions: A significant proportion of patients who had agreed a bone health management plan with the orthogeriatrician changed their mind following discharge from the hospital. Further studies are needed to understand the reasons for this as, although not proven in our small study, there are potential consequences of further fracture and complications. New initiatives are needed to encourage patients to comply with the plan for investigations and antiresorptive therapy.

\section{Abstract ID: 85 \\ Clinical audit/service evaluation \\ Subcategory: Management and treatment}

\section{The impact of delaying denosumab injections and choice of subsequent management on fracture rate}

\section{Jones, $K$ Lewis}

Rheumatology, City Hospitals Sunderland NHS Foundation Trust, Sunderland, UK

Background: Denosumab is a fully human monoclonal antibody used to treat Osteoporosis. Literature suggests patients who have delays between injections or those who have completed a course are at risk of rebound fractures. In post hoc analysis of the FREEDOM trial (Fracture Reduction Evaluation of Denosumab in Osteoporosis every 6 Months), authors recommend that those who discontinue denosumab should have an alternative anti-resorption treatment. ${ }^{3}$

Objective: The primary aim was to identify any link between delayed injections and fractures. The secondary aim was to evaluate if the choice 
of treatment after denosumab affected fracture rates.

Methods: Ninety patients who completed a course were analysed (two had incomplete data). The date of each injection was recorded, delays and reasons were noted. Follow on treatment and subsequent fractures were recorded.

Results: Sixty-two of 88 patients had at least one delayed treatment. Of 642 injections, 109 $(16.9 \%)$ were delayed, ranging from 1 to 36 weeks. In $65.1 \%$ no reason for delay was documented. The most common reason was blood abnormalities $(15.6 \%)$. Eleven patients sustained fractures. Ten experienced delays of varying length (215 weeks). Three were on Calcium/vitamin D, one on oral bisphosphonate and seven while on denosumab. Due to COVID-19, 57 patients had injections rescheduled. These were not included in the analysis.

Discussion: Fractures did not appear to correlate with longer delays. Four patients had fractures following completion of denosumab, $75 \%$ were not on anti-resorption treatment post denosumab. These numbers were not large enough to attach any statistical significance. Fracture rates were not analysed for the COVID-19 group as it is too early to capture the impact but this could done retrospectively in 18 months' time.

Conclusion: In this small patient cohort there was no correlation found between delays and occurrence of fractures. It appears that patients are more likely to fracture if not on anti-resorption treatment post denosumab but the numbers were too small to firmly conclude this. Further studies with larger numbers are required to robustly compare fracture rates and outcomes between groups receiving different anti resorption treatments.

\section{Abstract ID: 87 \\ Clinical audit/service evaluation Subcategory: Management and treatment}

\section{Sex hormone screening in men with established osteoporosis: a review of current practice}

\section{Lis, V Nadkarni, T Le, F Roberts, R Shahab,} M Osterdahl, F Martin

Perioperative Medicine for Older People Undergoing Surgery (POPS), Guy's \& St Thomas's NHS Foundation Trust, London, UK
Background: Hypogonadism has been identified in $5-15 \%$ of men with osteoporosis. ${ }^{1}$ As many as $50 \%$ of English Fracture Liaison Services (FLS) and $100 \%$ of Welsh FLS routinely screen for hypogonadism. ${ }^{2}$ Yet there is no evidence that treating hypogonadism reduces fracture risk or negates need for specific osteoporosis treatment. ${ }^{1}$

Objective: To review the clinical effectiveness of screening for hypogonadism in male patients under 70 years with confirmed osteoporosis by:

1. Establishing evidence base to support practice of sex hormone $(\mathrm{SH})$ testing (expert opinion, systematic review). This is reported in a separate abstract.

2. Auditing current practice - who are we screening? Is our screening practice robust? Does it change management?

3. Reviewing if there are more prevalent drivers of secondary osteoporosis present.

Methods: Retrospective analysis of the FLS-DB between September 2016 and October 2019 and manual review of electronic notes by two investigators of all men $\leqslant 70$ years with confirmed osteoporosis.

- Was SH testing completed appropriately (before $11 \mathrm{am}$, paired with sex hormone binding globulin (SHBG))?

- Did the result change management?

- Were other secondary causes more prevalent?

Results: 103 men aged $\leqslant 70$ were identified.

- 56/103 (54\%) had testosterone levels measured with abnormal results in 14/56 (25\%). Of those tested, only $26 / 56(47 \%)$ were performed before $11 \mathrm{am}$ and only 5/56 $(4.9 \%)$ had a paired SHBG.

- All 103 men were treated with anti-resorptive medications; the $\mathrm{SH}$ testing did not change management or prompt onward referral.

- This patient cohort has a higher prevalence of smoking ( $36 \%$ versus $\left.1.4 \%^{3}\right)$ and alcohol dependence $\left(44 \%\right.$ versus $\left.1.3 \%{ }^{4}\right)$ compared to national data.

Discussion: Our internal protocol lacks a robust evidence base. In turn, we are poorly adherent to it - the reasons for this have not been explored 
here but are likely varied. Where we are adherent, results of screening have not altered our practice. Local prevalence of smoking and alcohol dependency is high and may represent a more clinically effective target for risk factor modification in our cohort.

Conclusion: Our practice review has prompted a shift towards active screening for and treatment of alcohol and smoking dependence and away from routine screening for hypogonadism.

\section{References}

1. Hoppé E, Bouvard B, Royer M, et al. Is androgen therapy indicated in men with osteoporosis?. Foint Bone Spine 2013; 80: 459-465.

2. Royal College of Physicians, 2016. Fracture Liaison Service Database (FLS-DB) Facilities Audit. Royal College of Physicians, viewed 15 May 2020, <https://www.fffap.org.uk/fls/flsweb. nsf/docs/DownloadFiles/\$File/FLS-DB $\% 20$ facilities\%20audit\%20report_final_web. pdf?openelement>

3. UK Government, 2019. Alcohol dependence prevalence in England, UK Government, viewed 15 May 2020, <https:/www.gov.uk/government/ publications/alcohol-dependence-prevalence-inengland>

4. NHS Digital. https://digital.nhs.uk/data-andinformation/publications/statistical/statistics-onsmoking/statistics-on-smoking-england-2019

\section{Abstract ID: 89 \\ Clinical audit/service evaluation Subcategory: FLS}

\section{Community fracture liaison service: strategies to deliver specialist bone health consultant approved care in the community: a review}

\section{R Barbary, H Barnes, R Ramdewor}

Nottinghamshire County Fracture Liaison Service, East Bridgford Medical Centre, Nottingham, UK

Introduction: The Fracture Liaison Service (FLS) provides a specialist nurse led service in the community for patients over 50 years that have sustained a low traumatic fracture. This service utilises intravenous zoledronate as preferred first line choice of treatment. The service was set up as a pilot in 2015 through Vanguard funding and has significantly grown and changed over the last 5 years. New ways of working focus on patient centred care by delivering consultant approved management plans, within the community, through integrated shared care via Virtual Clinic.

Objective: The service aims to improve quality of life, avoid unnecessary hospital attendances in accordance with locally agreed pathways.

Method: Patients referred to FLS were reviewed between 01/01/2020 and 31/03/2020 and their treatment and intervention pathways were recorded.

Results: Virtual clinic reviews were collated $01 / 01 / 2020-31 / 03 / 2020$. These demonstrated large numbers of GP and clinic referrals. These referrals have led to 272 being assessed within the community, reducing the number of patients required to attend secondary care clinics.

167 approved for intravenous zoledronate 10 approved for subcutaneous denosumab 42 received 6 monthly repeat CTX or other blood monitoring

43 did not require bone sparing treatment and were given life style advice only

6 declined treatment

1 consideration of teriparatide

1 did not fit service criteria

2 known to bone health consultant with plan.

Discussion: Over 3 months FLS received 272 patients that were discussed with Virtual Clinic; and had treatment formalised and managed within the community. The FLS reviews, assesses, manages and systematically follows up patients. Investigations such as DXA and blood monitoring are requested and managed internally. The service continues to receive an increasing number of referrals from primary and secondary care and provides specialist guidance and support for effective treatment of bone health.

Conclusion: The FLS has led to 272 patients receiving specialist bone health treatment in the community. The service provides a secondary care consultant management plan within a primary care setting, decreasing attendance to secondary care clinics, consequently reducing time and costs. 


\section{Abstract ID: 90 \\ Clinical audit/service evaluation \\ Subcategory: Management and treatment}

\section{*Denosumab persistence across the secondary and primary care interface}

\author{
LA Statham 1,2,3, V Woodhall2,3, S Abdy², G Tobin², \\ S Oliver ${ }^{3}$, TJ Aspray² \\ 'School of Pharmacy, University of Sunderland, UK, ${ }^{2}$ The Bone Clinic, \\ Newcastle upon Tyne Hospitals NHS Foundation Trust, Newcastle, UK, \\ ${ }^{3}$ Pharmacy Directorate, Newcastle upon Tyne Hospitals NHS Foundation \\ Trust, Newcastle, UK
}

Background: Denosumab is a "Green Plus" formulary drug with initiation in secondary care and furtherdoses given in primary care. Discontinuation should be carefully considered, as the benefits of denosumab treatment wear off with a risk of rebound vertebral fracture.

Objectives: To identify whether denosumab prescriptions initiated in our hospital bone clinic have been continued in primary care. If discontinued: whether alternative treatment is in place and whether there have been any vertebral fractures.

Methods: Patients issued denosumab hospital prescriptions from January/2014 to March/2019 (or GP surgeries) were contacted to confirm on-going denosumab prescription. Data were evaluated in Microsoft Excel and patients categorised as being on/off denosumab or on/off alternative treatment.

Results: 610 patients were identified with a mean (range) age 74 (32-98) years, $88 \%$ female). 166 were excluded: deceased (120), not bone clinic/ never started denosumab (46). 352/444 (80\%) persisted with denosumab. Baseline T-scores were available for 258 patients: mean (range) femoral neck (FN), $-2.51(-0.5$ to -4.6$)$; total hip (TH) -2.32 , (+0.5 to -5.5$)$; lumbar spine (LS), -2.02 ( +4.8 to -5.1$)$. Mean T-scores for those continuing/discontinuing had been $\mathrm{FN}, 2.5 /-$ 2.54; $\mathrm{TH},-2.3 /-2.2 \& \mathrm{LS},-2.06 /-1.83$. Mean/ median (range) denosumab persistence was $34 / 30$ (12-66) months, but was less for those who stopped treatment: 15/6 (6-60) months for planned and 18/12 (6-60) months unplanned. Following discontinuation, 17 (18\%) were prescribed alternative therapy (oral bisphosphonate, strontium, zoledronic acid) and 75 were not [invited for review (45), patient choice (15), no suitable alternative (15)]. Adverse effects leading to discontinuation included felt unwell (9), fear of dental side-effects (1), skin conditions (3), nasal congestion (1), sciatica (1), Raynaud's (1), uveitis (1). One vertebral fracture following discontinuation was identified.

Discussion: Most patients, who typically had low BMD on initiation, persisted with denosumab. Of $20 \%$ who discontinued, half had no appropriate alternative, potentially increasing vertebral fracture risk, which may have been under-reported. Current improvements include: clear/concise clinic letters, treatment discussion and written information on denosumab (including discontinuation) to patients/GP.

Conclusion: Denosumab prescription mostly continued in primary care, but $10 \%$ patients experienced unsupervised discontinuation. Further work to improve effective and sustained prescribing between the patient, GP and secondary care continues.

\section{Abstract ID: 91 \\ Clinical audit/service evaluation Subcategory: DXA}

Extended femur scans for opportunistic identification of incomplete atypical femoral fractures: precision errors for hip bone mineral density and breaking measurements

\section{Smith', CW Wright², KM Knapp², A Black',} $R$ Hollick 2,3

'Department of Rheumatology, NHS Grampian, Aberdeen, UK, ${ }^{2}$ College of Medicine and Health, University of Exeter, Exeter, UK, ${ }^{3}$ Health Sciences Research Unit, University of Aberdeen, Aberdeen, UK

Introduction: The extended femur scan is new software introduced by GE to opportunistically identify incomplete atypical femoral fractures (iAFF) in people at an increased risk due to longterm bisphosphonate therapy. The clinical scan protocol includes a scan of the full length of femur, with the inclusion of a typical hip DXA scan for measurement of BMD in this region.

Objective: To investigate the in-vivo precision error of BMD and beaking index measurements of extended femur scans, in patients routinely attending for a DXA scan.

Methods: A short term precision study using extended femur scanning software was undertaken using same day duplicate scans in 30 participants with repositioning between scans. The root mean square standard deviation (RMSSD) and root 


\begin{tabular}{|c|c|c|c|}
\hline & $\begin{array}{l}\text { Total hip } \\
\text { BMD }\end{array}$ & $\begin{array}{l}\text { Neck of femur } \\
\text { BMD }\end{array}$ & Beaking Index (mm \\
\hline RMS SD & 0.011 & 0.015 & 0.473 \\
\hline RMS CV & 1.43 & 2.05 & 38.18 \\
\hline LSC (\%) & 3.96 & 5.68 & \\
\hline
\end{tabular}

mean square coefficient of variation (RMSCV\%) were calculated for BMD and the beaking index.

Results: Both sites indicate precision to be within the accepted figures quoted by the ISCD for $95 \%$ least significant change (LSC) for total hip 5.0\%, and femoral neck $6.9 \%$.

Discussion: The hip BMD precision errors are in line with published literature for standard hip DXA scans suggesting that there is no negative impact of using the extended femur scan hip measurements in clinical practice. The beaking index measurements yielded a much higher precision error in the femur, but this was in part due to two outliers. These were created by inaccurate placement of the cortical borders by the scan software and indicate a requirement to visually assess the lateral femoral cortex in conjunction with the scan software for indication of iAFF.

Conclusion: In clinical practice, visual assessment of the femoral cortex is required, alongside the beaking index to check the measurements and assess for iAFF.

\section{Abstract ID: 93 \\ Clinical audit/service evaluation Subcategory: Vertebral fracture}

\section{Using artificial intelligence technology to improve case finding for vertebral fractures in the fracture liaison service: a follow up}

\section{R Eckert ${ }^{1}, S$ Connacher ${ }^{1}, R$ Mansour ${ }^{2}$, PStevens' ${ }^{1}, G$ White ${ }^{1}$, P Osborne', J Kelly', $S$ Mahoney', C Milan', D Prieto-Alhambra ${ }^{3}$, $R$ Low' $^{1}$, MK Javaid ${ }^{1,3}$}

${ }^{1}$ Oxfordshire Fracture Prevention Service, Oxford University Hospitals, Oxford, UK, ${ }^{2}$ Radiology Department, Oxford University Hospital, Oxford, UK, ${ }^{3}$ NDORMS, NDORMS, Oxford, UK

Introduction: While opportunistic identification of patients with a vertebral fracture is recommended, the uptake and adherence to treatment recommendations in patients who have not presented with a fracture to an FLS are unclear.
Aim: To evaluate the impact of semi-automatic artificial intelligence technology for identification of vertebral fractures in the Fracture Liaison Service setting, focusing on patient experience and compliance.

Methods: Following the implementation of the Zebra AI system into the FLS setting, we reviewed the data of 50 patients who were positively identified as having an opportunistic vertebral fragility fracture to assess compliance with recommended treatments and also patient satisfaction of the care they received.

Results: Of 50 patients reviewed, average age 78 years, $66 \%$ were recommended treatment. $22 \%$ had died before their one-year assessment. Of those recommended treatments, $79 \%$ were compliant with their recommended medications at 1 year. No patients sustained a further fracture in the year after their consultation. Patient experience questionnaires sent after consultation had a $40 \%$ response rate. $100 \%$ would recommend the service to family \& friends.

Discussion: The long-term patient benefits from opportunistic vertebral fracture identification are unknown. This preliminary data confirm that patients identified in this way, accept and adhere to anti-osteoporosis medication. Further work is needed to explore methods to avoid treatments in patients with shorter survival.

Conclusion: The opportunistic identification of patients with vertebral fractures is associated with high patient initiation and adherence to anti-osteoporosis medication, but the mortality rate in this patient group was high.

\section{Abstract ID: 94 \\ Clinical audit/service evaluation Subcategory: Vertebral fracture \\ Improving knowledge of incidental vertebral column fractures amongst imaging requesters}

SA Anyan-Brown, E Watkins, H Morrin,
H Khalid, T Hinchliffe, F Martin
POPS Department, Guy's \& St Thomas' NHS Trust, London, UK

Background: Vertebral column fractures (VCFs) are a common incidental finding but there is no established terminology used to communicate 


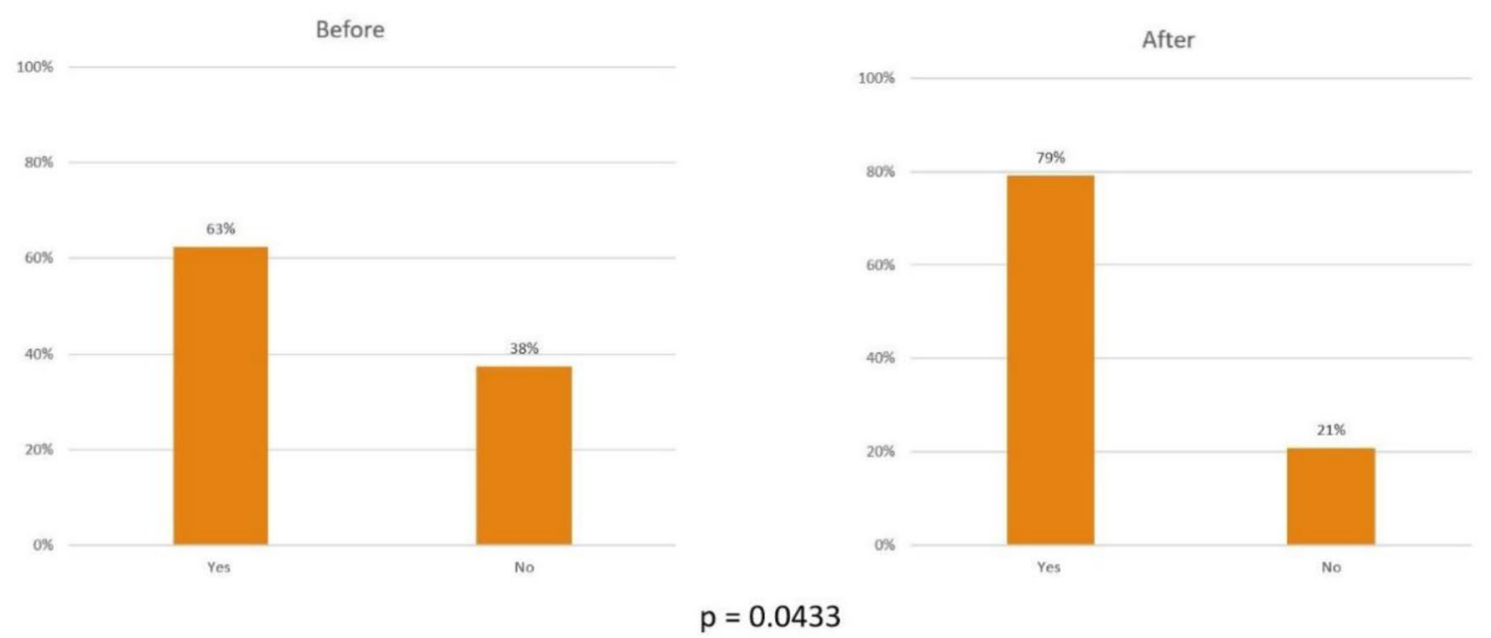

Figure 1. Responses from junior doctors $(n=48)$ before and after educational intervention to the question, “Would you be concerned if an asymptomatic incidental vertebral compression fracture was found on a scan?"

them in reports. They are therefore often missed. Locally, we use a direct referral from radiology to our Fracture Liaison Service (FLS) to mitigate this. However this is likely to contribute to deskilling of imaging requesters who no longer need to identify VCFs described in various ways on imaging reports. We sought to design a user friendly educational tool to counterbalance this.

Aims and Objectives: Improve knowledge and awareness of incidental VCFs amongst imaging requesters.

\section{Objectives:}

1. Measure current awareness of significance of incidental VCFs

2. Design and implement a short educational intervention to improve awareness

3. Reassess for improvement

Methods: Plan-Do-Study-Act methodology used to assess awareness using questionnaires before and after a 3-minute teaching intervention delivered in October and November 2019. Data was collected from 48 junior doctors and categorical data was statistically analysed using McNemar's Chi-Squared Test.

Results: Good baseline awareness of the significance of VCF (63\%) which increased after educational intervention (79\%). This lead to a greater proportion of junior doctors considering onward referral to FLS/osteoporosis clinic (60-92\%) and knowing local referral pathway (17-100\%).
Discussion: This study showed a simple educational intervention significantly improved awareness of different terminology used to describe $\mathrm{VCF}$ and what to do if this is reported.

Conclusion: Our next steps are to continue delivering departmental presentations. This coming academic year we intend to incorporate teaching into the FY1 timetable and expand sessions to ensure coverage to registrar level. This will need to be done creatively using virtual platforms due to COVID era restrictions.

\section{Abstract ID: 95 \\ Clinical audit/service evaluation Subcategory: DXA}

\section{Outcome of bone density assessment in patients on aromatase inhibitors: applying the international recommendations of $\mathbf{2 0 1 7}$ in practice}

\section{S Onuekwa', O Olajobi', J Bradley², S Smitten², S Venkatachalam \\ ${ }^{1}$ Cannock \& Wolverhampton Rheumatology Centre, The Royal Wolverhampton Hospital NHS Trust, Wolverhampton, UK, ${ }^{2}$ Dept of Medical Physics, The Royal Wolverhampton Hospital NHS Trust, Wolverhampton, UK}

Background: Patients on aromatase inhibitors have rapid bone loss and increased of risk of fragility fractures and the duration of their use has gone up to 10 years. The new international recommendations for management of aromatase inhibitor-induced bone loss from IOF and other 
organisations in March 2017 differs from the UK expert consensus recommendations from 2008.

Objectives: Outcome of bone density assessment on patients on aromatase inhibitors after the new international recommendations in March 2017.

Methods: All patients on an aromatase inhibitor who had a bone density scan from October 2018 to March 2019 were analysed for their demographics, clinical risk factors and bone density values from Lumbar spine and Femoral Neck/Total Hip.

Results: 200 patients on aromatase inhibitors had undergone a DXA scan in the 6-month study period. The patients were predominantly Caucasian $(94 \%)$ with a mean age of 67.9 years. Their mean BMI was $29.2 \mathrm{~kg} / \mathrm{m}^{2} .93$ patients had their first DXA on AI and have been on treatment for a mean duration of 11.2 months. 107 patients had a repeat scan and were on AI for a mean duration of 37.2 months. 52 patients had a $\mathrm{T}$ score $<-2.0$ at the hip or spine and 31 patients had a $\mathrm{T}$ score $<-1.5$ at the hip or spine and 2 clinical risk factors warranting intervention. The BMD report was in agreement with new international recommendations in $89 \%$ but differed in 22 patients. It was mainly because of history of previous smoking documented but not accounted as a risk factor.

Discussion: There is still a delay in patients on aromatase inhibitors being referred for a DXA scan. History of previous smoking also can increase fracture risk in patients on aromatase inhibitors and has to be taken into account in recommending treatment.

Conclusion: We need to increase awareness of the new international recommendations for the management of aromatase inhibitor-induced bone loss to avoid delay in referral.

\section{Abstract ID: 96 \\ Clinical audit/service evaluation Subcategory: COVID}

\section{Challenge of denosumab treatment for osteoporosis patients in the COVID pandemic in UK}

A Leong, K Salt, J Dodd, S George, H Sapkota, $S$ Venkatachalam

Cannock \& Wolverhampton Rheumatology Centre, The Royal Wolverhampton NHS Trust, Wolverhampton, UK
Background: Osteoporosis services were impacted by the COVID pandemic as the UK was in lockdown from 23 March 2020. Nearly 300 of our 900 osteoporosis patients on denosumab were due for their next injection and some of them were unable to attend hospital as they were shielding. A delay of more than 4 weeks in denosumab delivery can result in rebound bone loss and vertebral fractures.

Objective: To ensure patients with osteoporosis, do not miss their treatment with denosumab and to enable self-administration or another alternative, to avoid hospital visits during the COVID19 pandemic.

Methods: Osteoporosis patients who were due their denosumab between 25 March 2020 and 24 June 2020 were contacted. They were offered the choice of self-administration or switching to an oral bisphosphonate. Denosumab was sent via a local courier service with a website link for education. The patients who were unable to selfadminister were prescribed either, ibandronate once monthly or alendronic acid once weekly for 3 months, if they had no contraindication. Patients who were not shielding attended our day unit at the cold site for their denosumab injection.

Results: 137 patients had Denosumab home delivery and 124 of them self-administered while 13 patients had district nurse support. 70 patients attended the day unit and 66 of them had denosumab and four were switched to zoledronic acid. 79 patients received oral ibandronate for 3-6 months.

Discussion: We had to plan this at short notice after lockdown and were unable to get blood tests in most patients, but they all had calcium and Vitamin D supplementation. UK, European and American guidelines which came out later support our pragmatic practice during the pandemic. We opted for ibandronate once monthly as an alternative as it can be given in patients with CKD.

Conclusion: COVID-19 pandemic has adversely impacted routine delivery healthcare services, especially to osteoporosis patients. Our experience has empowered most of our patients to selfadminister denosumab in the middle of a health crisis. We plan to extend this practice as we restore our services. 


\section{Abstract ID: 97 \\ Clinical audit/service evaluation \\ Subcategory: FLS}

\section{How has reconfiguring a fracture liaison service changed fragility fracture patients' management?}

\section{J Fleming ${ }^{1,2}$, K Blesic 1, P Gorman' 1 K Tharp $1, G$ Darnell1,3, M Young1,3, H Bebbington 1,3, K Wynn', $R$ Summers ${ }^{5}$, C Chan $^{5}, M_{\text {Vindlacheruvu }}^{6}$ \\ ${ }^{1}$ Fracture Liaison Service, Addenbrooke's Hospital, Cambridge, UK, 2Public Health \& Primary Care, University of Cambridge, Cambridge, UK, ${ }^{3}$ Physiotherapy, Addenbrooke's Hospital, Cambridge, UK, "Radiology, Addenbrooke's Hospital, Cambridge, UK, ${ }^{5}$ Rheumatology, Addenbrooke's Hospital, Cambridge, UK, ${ }^{\circ}$ Medicine for the Elderly, Addenbrooke's Hospital, Cambridge, UK}

Background: Fracture Liaison Services play a key secondary prevention role by systematically identifying $\geqslant 50$-year-old patients with fragility fractures, then investigating, offering information and making appropriate treatment recommendations/referrals. Our hospital previously employed a fracture liaison nurse using research funds. A patient focus group highlighted service development priorities, including more time for patient education, more follow-up regarding medication and more information on exercise for better balance and bone health. This guided our business case for a full commissioned service.

Objective: To track 3 years' service changes and understand their impact on outcomes

Methods: Descriptive analyses of routinely collected data

Results: From April 2017 to March 2020 the team has grown to five (3.2 full-time-equivalent), including a part-time musculoskeletal physiotherapist. Previously the nurse tried to see fragility fracture patients attending fracture clinic. Still outpatient-based, the team now identifies from daily trauma lists, including in-patient admissions and emergency department discharges. We refer all 50/75-year-olds with fragility fractures for bone densitometry in line with NICE guidance and invite to a 'bone health assessment' clinic only those with osteoporosis or osteopaenia along with a high fracture risk score. We aimed to conduct these osteoporosis and fall risk assessments with $>75$-year-olds attending for orthopaedic follow-up but also developed templates with guideline recommendations for referring to their
GP those we missed seeing. Fragility fracture identification has increased (2017/18: $n=733$, 2019/20: $n=1633$, up $123 \%$ ), particularly amongst $>75$-year-olds (from $27 \%$ to $48 \%$ of $2017 / 18$ and $2019 / 20$ totals respectively). Our initial contacts with these older patients has become usually by letter with fracture risk assessments included at follow-up if no in-patient assessment. Crucially, we currently reach near$100 \%$ follow-up in these new 4-month and 12-month post-fracture telephone clinics and $>2$-fold higher than average medication adherence. Weekly multidisciplinary team meetings facilitate regular review of complex cases with orthogeriatrics, metabolic bone and radiography input. Our FLS physiotherapist runs a 5-week $1 \frac{1}{2} \mathrm{~h}$ group programme with team input on patient education sessions. Evening education sessions in local venues 3 times/year offer further information about osteoporosis, bone health and falls prevention.

Discussion/Conclusion: FLS-Database audit confirms above national average outcomes, but gathering local data enables learning how context and implementation can help achieve improvements.

\section{Abstract ID: 98 \\ Clinical audit/service evaluation Subcategory: Vertebral fracture}

\section{Under-reporting of vertebral fractures in CT scans: a missed opportunity for preventing osteoporotic fractures}

\section{S Byravan', E Comben², D Davda², W Pang², H Sapkota', S Venkatachalam \\ ${ }^{1}$ Cannock \& Wolverhampton Rheumatology Centre, The Royal Wolverhampton NHS Trust, Wolverhampton, UK, ² Dept of Radiology, The Royal Wolverhampton NHS Trust, Wolverhampton, UK}

Background: After an initial vertebral fracture, the subsequent risk for another fracture is $25 \%$ in the first year. Vertebral fragility fractures are often left unreported during routine radiological examination.

Objectives: To assess local practice in identification and reporting of vertebral fragility fractures as part of a national audit of the Royal College of Radiology and identify the clinical characteristics of these patients. 
Methods: An audit of CT-TAPs in patients over the age of 70 years over a 3-week period in January 2019 was carried out from the Radiology Reporting System. The reformatted CT images were reviewed, and the vertebral fractures were graded by the semi-quantitative method of Genant. Patients with bone metastases and severe trauma were excluded. Electronic medical records of the patients were reviewed to identify the underlying diagnosis, previous DXA scan, ongoing osteoporosis treatment, previous fragility fractures and relevant comorbidities.

Results: There were 105 CT-TAPs performed during the audit period. 14 patients were excluded because of bony metastases; 18 of the remaining 91 patients had moderate/severe vertebral fragility fractures. Five of them had all the vertebral fractures identified in the initial report, four patients had some but nine did not have any reported. None of the scans included an appropriate recommendation for further investigation for osteoporosis. Five patients had a previous fragility fracture, one had a recent DEXA scan and only two patients were on bisphosphonates. There were multiple comorbidities including previous cancer in five and concurrent cancer in nine patients.

Discussion: Identifying those patients with moderate/severe vertebral fragility fractures provides an opportunity to treat osteoporosis. This can prevent more disabling future fractures that can have a significant impact on patient's morbidity and quality of life.

Conclusion: Increasing awareness of the incidental vertebral fragility fractures in the reporting radiologists and a direct referral pathway to the local fracture liaison service is recommended.

\section{Abstract ID: 99 \\ Clinical audit/service evaluation Subcategory: Vertebral fracture}

\section{Does routine VFA improve vertebral fracture identification and improve osteoporosis treatment?}

\footnotetext{
A Reynolds', S Smitten², S Naylor², J Bradley², H Sapkota', S Venkatachalam'

${ }^{1}$ Cannock \& Wolverhampton Rheumatology Centre, The Royal Wolverhampton NHS Trust, Wolverhampton, UK, 2Dept of Medical Physics, The Royal Wolverhampton Hospital NHS Trust, Wolverhampton, UK
}

Background: Vertebral fractures are the most common type of osteoporotic fracture, yet majority are not clinically recognised. Vertebral Fracture Analysis (VFA) is a diagnostic tool with the potential to significantly improve vertebral fracture identification with significantly reduced radiation dose compared to standard radiography.

Objective: To identify the incidental vertebral fractures and the number of patients advised osteoporosis treatment following use of VFA when undertaking a dual-energy X-ray absorptiometry (DXA) scan.

Methods: We had a retrospective audit over 3 months of 1354 DXA scans and $43 \%$ fulfilled criteria for VFA. This was followed by a month of prospective study with all adult patients ( $\geqslant 18$ years) referred for BMD measurement stratified against the International Society for Clinical Densitometry (ISCD) criteria for VFA (ISCD, 2019). Those fulfilling the criteria underwent a VFA using Genant semiquantitative classification, in addition to the axial DXA scan. Patients were recommended treatment based on FRAX $^{\circledR}$ score or the presence of a Grade 2 vertebral fracture as per UK National Osteoporosis Guideline Group (2017) recommendations.

Results: A total of 202 patients underwent a DXA scan, with 88 patients (43.6\%) meeting the ISCD criteria for VFA. Of the 629 evaluable vertebrae a total of 71 vertebral fractures $(11.3 \%)$ were identified (Grade 1 or above) in $58.3 \%$ of patients. Patients with one or more VFs had a significantly lower BMD than patients without a vertebral fractures $(p=0.005) .86$ patients $(44.1 \%)$ had a change in management, a relative increase of $24.6 \%$ utilising stratified VFA combined with routine DXA.

Discussion: ISCD criteria are highly effective at identifying the high-risk patients as one in two patients were identified to have a vertebral fracture of at least Grade 1 or above. VFA led to a $25 \%$ increase in the number of patients receiving treatment for osteoporosis.

Conclusion: Routine VFA with DXA when indicated was more effective at screening patients for prevalent vertebral fractures. We plan to extend VFA analysis as a routine with increased time allotted for DXA scan. 


\section{Abstract ID: 100 \\ Clinical audit/service evaluation \\ Subcategory: Vertebral fracture}

The acute spine fracture in the emergency department; a retrospective review

o Thorpe, A Beirne, P Fox, A Nic Uidhir, FDockery

Geriatric Medicine, Beaumont Hospital, Dublin, Republic of Ireland

Background: As to who should manage osteoporotic spine fractures presenting to Emergency departments (ED) is sometimes debated. We sought to review practice regarding their management in our institution, that might inform a clinical pathway.

Methods: We conducted a search of radiology reports for a consecutive series of thoracic or lumbar spine $\mathrm{x}$-rays ordered by ED team only, that is, a series of patients whose presenting complaint was a suspected acute fracture. We narrowed search to include terms compression or wedge or end-plate.

Results: Over 7 months, there were 1505 such reports; narrowed search and excluding duplicates yielded 168 patients of whom 84 had a fracture. We looked at the acute management of those $>50$ years, excluding one metastatic fracture leaving $n=64$. Of these, $65 \%$ occurred following a fall, $14 \%$ on twisting/bending/coughing, $14 \%$ spontaneous, $7 \%$ unclear onset. ED first consulted orthopaedics for 10 cases, neurosurgeons for 2, physicians for 18. A total of 21 were discharged from ED (five having speciality review pre-discharge). A further 11 were sent home from ED with fracture diagnosis made only when x-ray subsequently reported. Of those admitted, 28 went to physicians with consult to surgeons in $39 \%$, two went to orthopaedic surgery, two to other specialists. Admission was complicated by pressure ulcer in 13\% (4/32), pneumonia in 13\%. Overall 24/64 (38\%) went on to have MRI/CT (mainly admitted cases). At least 12 were managed with a brace (all records not available). $n=7$ $(11 \%)$ had later vertebroplasty. More than half had no documented osteoporosis treatment plans nor GP instruction to address.

Conclusion: Care of spine fractures presenting acutely varies; a high proportion managed by ED solely. Whether outcomes vary as a result is not answered by this audit but there is a need for a pathway to inform best practice. Osteoporosis is inadequately-addressed in this high risk group, highlighting need for fracture liaison services in post-acute management 


\section{Original Research}

All abstracts marked with * received commendations

\section{Abstract ID: 2 \\ Original Research \\ Subcategory: Management and treatment}

\section{Experiences of falls in domestic settings and use of ambulance services: an ethnographic study}

\section{Heaton ${ }^{1,4,5,6}$, T Williamson' ${ }^{1}$ J Leigh'2,} E Collier ${ }^{3}$

${ }^{1}$ Family care \& Dementia studies, University of Worcester, Worcester, UK, ${ }^{2}$ Nurse Education Practice, University of Salford, Manchester, UK ${ }^{3} \mathrm{H}$ ealth and Social Care Research Centre, University of Derby, Derby, UK, "Bridgewater Foundation NHS Community Trust, Greater Manchester, UK, ${ }^{5}$ NorthWest Ambulance Service, Greater Manchester, UK, ${ }^{6}$ Wrightington, Wigan \& Leigh Foundation NHS trust, Wigan, UK

Introduction: Each year, 35\% of people over age 65 years experience falls and approximately $45 \%$ of those are aged over 85 and live in the community. Local unpublished data from an English ambulance service found that following ambulance callouts, $24-32 \%$ of the patients were not conveyed to hospital and of those, only $32-52 \%$ were referred to the local Falls Prevention Service for a specialist multifactorial assessment (NICE, 2013).

Background: The literature review undertaken supports the need for a falls pathway (guidance for practitioners) to be in place which is fully utilised, by all practitioners. However, it did not uncover the reasons for low referral rates of non-conveyed patients to Falls Prevention Services. This thesis will outline the design of a study which aims to, gain an in-depth understanding of the falls patient journey from patient and ambulance crew perspectives and generate a clear understanding of the ambulance service customs and practices which could inform improvement of the existing falls pathway.

Methods: A critical ethnographic approach enabled participants' values, behaviours and beliefs to be explored. The methods were participatory observation, semi-structured interviews of patients/carers and ambulance crew and in-depth field notes. The sample was people over 50 years of age who had fallen or their carers $(n=10)$ who had been seen by the ambulance service. Ambulance crew were also recruited $(n=10)$.

Results: The study gained an in-depth understanding of the experiences of crew and patients/ carers. For both ambulance crew and patients/ carers, there are two shared themes 'falls journey' \& 'falls not being a problem for patients'. For crew 'training' was a theme and for patients/carers 'language' and 'patient transport' are themes. The study's unique contribution is that it has gained an in-depth understanding of the patient journey from patient and ambulance crews' perspectives and a better understanding of the fall's pathway. In summary, the falls pathway in the local area was clearly understood and followed by all crew observed. The study shared the challenges of recruiting in a hard-to-reach group.

\section{Abstract ID: 7 \\ Original Research \\ Subcategory: MSK}

\section{How does radiographic knee osteoarthritis impact musculoskeletal aging in midlife? Findings from the Hertfordshire Cohort study}

NR Fuggle, MA Clynes, KA Jameson, C Cooper, EM Dennison

MRC LEU, University of Southampton, Southampton, UK

Objectives: Osteoarthritis (OA) is the most common joint condition and can be defined clinically or radiologically. The aim of this study was to investigate whether a diagnosis of radiological knee OA impacted involution in muscle or bone in the midlife in a group of community-dwelling older adults in the UK.

Materials and methods: Our study comprised 220 members of the Hertfordshire Cohort Study (118 males and 102 females), a group of community dwelling older adults in the United Kingdom. Knee radiographs were performed at baseline, with osteoarthritis defined as a Kellgren and Lawrence score $\geqslant 2$. At baseline and follow-up included questionnaires to assess physical activity and lifestyle factors, JAMAR dynamometry, eight foot walk test and DXA, both of whole body and hips. Linear regression was performed to analyse associations before and after adjustment for follow-up time, lifestyle factors and anthropometry 
and the development of hand osteoarthritis was adjusted for grip strength outcomes.

Results: The mean age of participants at baseline was 65.0 years. Median follow-up was 16.7 years (range 15.0-18.4 years). Knee osteoarthritis was present in 75 (34\%) participants.

Radiographic knee osteoarthritis status at baseline was significantly associated with grip strength $(\beta=-3.2 \mathrm{~kg},-5.3$ to $-1.1, p<0.01)$ at follow-up in females but not in males $(\beta=-2.9,-5.8$ to 0.1 , $p=0.06)$.

No significant associations between radiographic osteoarthritis status at baseline and other measures of sarcopenia (gait speed or lean mass) or bone mineral density were found in either sex.

Conclusions: Our findings demonstrate that the presence of knee osteoarthritis in midlife can have a substantial bearing on grip strength in women over 15 years later, independent of the presence of hand osteoarthritis. Grip strength has been shown to correlate closely with lower limb strength and so the demonstrated association between grip strength and knee osteoarthritis is representative of a global weakness resulting from the disease. Thus, in order to countermand this association, it may be that concerted interventions (including physical therapy, strengthening and surgical intervention) should be recommended for those with knee osteoarthritis at this stage in the life course. Of course, these findings require replication in other cohorts.

\section{Abstract ID: 10 \\ Original Research \\ Subcategory: Hip fracture}

\section{*Incidence and predictors of hip fracture in patients with intellectual disabilities}

\footnotetext{
V Frighi' ${ }^{1}$ MC Smith², TM Andrews ${ }^{3}$, GS Collins ${ }^{4}$, J Roast ${ }^{5}$, L Clifton $^{6}$, A Fuller ${ }^{2}$, TA Holt ${ }^{2}$

${ }^{1}$ Deptartment of Psychiatry, University of Oxford, Oxford, UK, ${ }^{2}$ Nuffield Department of Primary Care Health Sciences, University of Oxford Oxford, UK, ${ }^{3}$ Learning Disabilities, Oxford Health NHS FT, Oxford, UK, ${ }^{4}$ Centre for Statistics in Medicine, University of Oxford, Oxford, UK,

${ }^{5}$ Learning Disabilities, Dimensions UK, Oxford, UK, ${ }^{6}$ Nuffield Department of Population Health, University of Oxford, Oxford, UK
}

Background: In current guidelines, patients with intellectual disabilities (ID), including Down's Syndrome (DS) do not figure as at high risk of osteoporosis, despite some evidence of increased fracture rates and decreased bone mineral density.

Objective: To investigate the incidence and predictors of hip fractures in patients with ID.

Methods: All patients aged 30-79 with ID in a Primary Care database (Clinical Practice Research Datalink) with linkage to the Hospital Episode Statistics were included. They were followed for maximum 10 years or until their first new fracture. Hazard ratios (HR) for potential fracture predictors were estimated using multivariable Cox regression. An age and sex matched cohort without ID was extracted for comparison.

Results: 39,073 patients were identified, of whom 3,915 with DS. Median (10th-90th percentile) age at entry was $39.2(30-61.8)$ years. $56.7 \%$ were men, $20.6 \%$ had epilepsy (vs $1.2 \%$ in the controls). In patients with ID and controls respectively, incidence rate $(95 \% \mathrm{CI})$ of major osteoporotic fracture (vertebra, wrist, shoulder, hip) was 45.7 (43.0-48.5) versus 24.2 (22.326.3)/10,000 person-years and of hip fracture $15.6(14.0-17.3)$ versus $4.6(3.8-5.5) / 10,000$ person-years. Hip fracture represented $35.8 \%$ of all major osteoporotic fractures in the ID versus $22.4 \%$ in the control group. Independent predictors of hip fracture [HR (95\% CI)] within the ID group included age [2.31 (2.06-2.60) in females, $2.08(1.86-2.33)$ in males for each ten-years increase]; previous osteoporotic fracture [1.89 (1.37-2.61)]; previous other fracture [1.44 (1.081.91)]; history of falls [1.75 (1.26-2.43)]; epilepsy $[1.89(1.49-2.40)]$; diabetes $[1.55$ (1.08-2.23)]; Down's syndrome [1.93 (1.302.87)]. Higher BMI predicted lower risk [0.79 $(0.70-0.89)$ for each 5 points increase].

Discussion: These results, from the largest cohort of ID and DS patients ever studied, show that their hip fracture rate is 3.4 times higher than their general population counterparts. A diagnosis of Down's Syndrome confers a particularly high risk in the ID group.

Conclusion: Patients with intellectual disabilities are at high risk for major osteoporotic fractures, particularly hip fractures. Current guidelines should be revised, and measures introduced to address bone health and reduce fracture risk in this population 


\section{Acknowledgement/disclaimer}

This abstract presents independent research funded by the National Institute for Health Research (NIHR) under its Research for Patient Benefit (RfPB) Programme (Grant Reference Number PB-PG-1216-20017). The views expressed are those of the authors and not necessarily those of the NIHR or the Department of Health and Social Care.

We would like to acknowledge the Royal Osteoporosis Society, the Royal Mencap Society, and the Down's Syndrome Association as our research partners.

\section{Abstract ID: 14 \\ Original Research \\ Subcategory: Vertebral fracture}

Concurrent and predictive validity of visual approaches to densitometric vertebral fracture assessment: a systematic review of the literature

\section{N Powrie', C Richards² \\ 'Department of Radiology, Salford Royal NHS Foundation Trust, Salford, UK, 2University of Derby, Derby, UK}

Background: Vertebral fractures are associated with future fracture, mortality, and decreased quality of life, therefore are strong indicators for osteoporosis therapies. ${ }^{1}$ Research on conventional radiographs has suggested the Algorithm Based Qualitative approach (ABQ) to diagnosis may be superior to the Semi-Quantitative (SQ) approach in terms of association with Bone Mineral Density (BMD) and future fracture. ${ }^{2}$ Vertebral fracture assessment (VFA) is an image of the spine, obtained on a Dual-energy X-ray Absorptiometry (DXA) scanner, to diagnose vertebral fractures, as a low radiation dose and convenient alternative to conventional radiographs in patients undergoing DXA. The optimal diagnostic approach on VFA remains unclear.

Methods: A systematic review of the literature was conducted to identify studies evaluating strength of association with BMD and future fracture (concurrent and predictive validity) of either approach. Searches of the AMED, EMBASE, MEDLINE and CINAHL electronic databases, in addition to manual searches of reference lists and journal contents identified eleven relevant studies.
Results: Similar strength of evidence was identified for both approaches for concurrent validity, for all grades of fracture, and moderate-severe fractures only, for the SQ approach. There was some evidence of concurrent validity for mild fractures for the ABQ, but not SQ approach, based on one post-hoc analysis. There were similar levels of evidence for predictive validity for all grades of fracture and moderate-severe fractures, for both approaches. There is no evidence mild fractures identified using either approach are independent predictors of future fracture, however it is probable studies were underpowered for such a sub-group analysis.

Discussion: No comparative studies were identified; it cannot be concluded one diagnostic approach is superior. It is probable mild vertebral fractures are the major difference between approaches, however, comparative studies are required.

Conclusion: Evidence for concurrent and predictive validity of the $A B Q$ and $S Q$ approaches is of similar strength. Further comparative studies are required to define the optimal diagnostic approach.

\section{References}

1. Kendler DL, Bauer DC, Davison KS, et al. Vertebral fractures: clinical importance and management. Am F Med 2016; 129: e1-e10.

2. Lentle B, Koromani F, Brown JP, et al. The radiology of osteoporotic vertebral fractures revisited. F Bone Miner Res 2019; 34: 409-418.

\section{Abstract ID: 15 \\ Original Research \\ Subcategory: Vertebral fracture}

\section{*Does thoracic kyphosis severity predict response to physiotherapy rehabilitation in patients with osteoporotic vertebral fracture?}

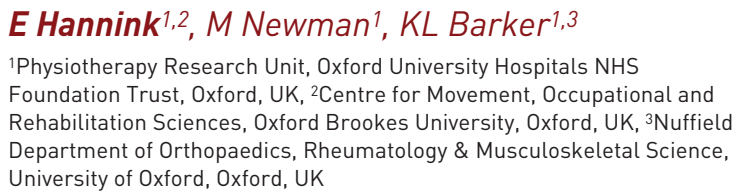

'Physiotherapy Research Unit, Oxford University Hospitals NHS Foundation Trust, Oxford, UK, ${ }^{2}$ Centre for Movement, Occupational and Rehabilitation Sciences, Oxford Brookes University, Oxford, UK, ${ }^{3}$ Nuffield Department of Orthopaedics, Rheumatology \& Musculoskeletal Science, University of Oxford, Oxford, UK

Background: Symptoms of osteoporotic vertebral fracture (OVF) and hyperkyphosis include 
pain, limitations in physical function, reduced activities of daily living and social participation, and restrictive pulmonary function. Hyperkyphosis and OVF both typically progress with age, and while they can be overlapping in their presentation, they are not synonymous physiological phenomena. Increased kyphosis angles in people with and without osteoporosis have been associated with decreased gait and balance stability.

Objective: To explore the relationship between kyphosis severity and physical function measures and to investigate whether kyphosis severity predicts response to physiotherapy intervention in people with OVF.

Methods: Secondary data analysis of the PROVE trial, a multicentre randomised controlled trial. Data were divided into severity quartiles of baseline thoracic kyphosis. Linear mixed models estimated the effects of kyphosis severity and time point on changes in kyphosis, Six-minute walk test (6MWT), Functional reach (FR), and Short Performance Physical Battery (SPPB).

Results: Of 604 participants, the moderately and severely hyperkyphotic reduced their kyphosis angle at 4 months and maintained improvement at 12 months (3.02 and 8.04 degrees, respectively), adjusting for age, height, back extensor endurance, and type of physiotherapy intervention received. FR scores were lower in the severely hyperkyphotic compared to normal kyphosis quartiles Q1 and Q2 (3.0 cm (95\% CI 1.1, 4.9) and $2.3 \mathrm{~cm}$ (95\% CI 0.4, 4.1), respectively). $6 \mathrm{MWT}$ scores were lower in the severely hyperkyphotic compared to the normal kyphosis group (Q2) by 39.63 metres (95\% CI 8.77, 70.49). SPPB scores were lower in the severely hyperkyphotic compared to the hypo/normal kyphosis group (Q1) by 0.72 points (95\% CI $0.14,1.31$ ), and all quartiles cumulatively improved at 4 months (0.42 (95\% CI $0.24,0.60)$ and 12 months $(0.25$ (95\% CI $0.05,0.45)$ compared to baseline.

Discussion: Results suggest that people with OVF(s) and severe hyperkyphosis can improve both spinal curvature and physical performance after a physiotherapy intervention.

Conclusion: People who have the most affected thoracic curvature can benefit the most from physiotherapy.

\section{Abstract ID: 17 \\ Original Research \\ Subcategory: MSK}

Are matrix metalloproteinase breakdown products associated with musculoskeletal health in older male adults? Findings from the Hertfordshire Cohort Study

NR Fuggle, HS Morgan, MA Clynes, KA
Jameson, P Titcombe, C Cooper, EM Dennison
MRC Lifecourse Epidemiology Unit, University of Southampton, UK

Objectives: Matrix metalloproteinases (MMPs), responsible for collagen degradation, have been detected in osteoblasts, osteocytes, osteoclasts, and chondrocytes in bone tissue, where they are known to perform specific functions. Furthermore, they play an important role in the homeostasis and maintenance of myofibre functional integrity in skeletal muscle. We therefore aimed to identify whether there was an association between MMPbreakdown products and musculoskeletal health in older adults from a community dwelling UK cohort.

Materials and methods: 259 male participants in the Hertfordshire Cohort Study (HCS) attended a study clinic where a lifestyle questionnaire was administered and fasting blood samples were taken and tested for MMP breakdown products [MMP-degraded type 1 collagen $(\mathrm{C} 1 \mathrm{M})$, MMP-degraded type III collagen (C3M) and citrullinated and MMP-degraded vimentin (VICM)]. We investigated associations between levels of MMP breakdown products and history of previous fall or fracture. Grip strength was measured using a Jamardynamometer. Bone mineral density (BMD) at the total hip and lumbar spine was measured in a subset using a Hologic QDR 4500 instrument. Linear regression was used to examine associations in both unadjusted models and those adjusted for age, BMI, social class, smoker status, alcohol consumption, physical activity score and dietary calcium intake.

Results: Mean age of study participants was 65.6years $(\mathrm{SD}=2.9)$ and median $\mathrm{BMI}$ was $26.9 \mathrm{~kg} / \mathrm{m}^{2}(\mathrm{IQR}=24.6-29.4)$. Median values of MMP breakdown products were $49.2 \mathrm{ng} / \mathrm{ml}$ $(\mathrm{C} 1 \mathrm{M}), \quad 22.0 \mathrm{ng} / \mathrm{ml} \quad(\mathrm{C} 3 \mathrm{M})$ and $2.20 \mathrm{ng} / \mathrm{ml}$ (VICM). There was no significant association between MMP breakdown products and BMD, or history of previous falls or fracture. A weak 
negative association was found between $\mathrm{C} 1 \mathrm{M}$ and grip strength ( $\beta$ coefficient $=-0.020(95 \%$ confidence interval $=-0.039,-0.002), p=0.031$ ) but this was not maintained after adjustment for confounders.

Conclusions: We found no association between MMP breakdown products and musculoskeletal health in this male cohort after adjustments. Studies in women are, however, warranted.

\section{Abstract ID: 19 \\ Original Research \\ Subcategory: Fracture risk}

Fragility fractures, falls, osteoporosis and their predictors in 20693 South Asian adults aged over 50 years: analysis of UK primary care data

\section{AL Darling', S de Lusignan',3}

${ }^{1}$ Nutrition, Food \& Exercise Sciences, University of Surrey, Guildford, UK, ${ }^{2}$ Nuffield Department of Primary Care Health Sciences, University of Oxford, Oxford, UK, ${ }^{3}$ Research and Surveillance Centre, Royal College of General Practitioners, UK

Background: There is a lack of data on falls, fractures and osteoporosis in western dwelling South Asian populations.

Objective: We assessed how many South Asian adults over the age of 50 years are diagnosed with fragility fractures $(\mathrm{FF})$, falls and osteoporosis, as well as the factors predicting these outcomes and the treatment given by General Practitioners.

Methods: We analysed routinely collected, pseudonymised patient data from the UK Royal College of General Practitioners (RCGP) Research and Surveillance Centre primary care network, including all data up to 1st January 2019.

Results: For females and males combined ( $n 20$ $693), 19 \%$ had a previous fall, $6.2 \%$ had a previous $\mathrm{FF}$ and $11.2 \%$ had osteoporosis. Very few $(1-2 \%)$ had been coded as having been offered osteoporosis specific health, diet or exercise advice. Forty-six per cent had been prescribed a calcium and vitamin D combined supplement, and $8 \%$ prescribed a calcium only supplement. Some form of vitamin D intake or deficiency assessment was common (30.6\%, with $13.4 \%$ having at least one 25 -hydroxyvitamin D assessment). Logistic regression analysis showed an increased odds of $\mathrm{FF}$ with increased age
$(\mathrm{OR}=1.05 ; 95 \% \mathrm{CI} 1.04-1.05)$ as well as reduced odds with male gender $(\mathrm{OR}=0.45$; 95\% CI 0.39 0.51 ) and increased body mass index (BMI) $(\mathrm{OR}=0.98 ; 95 \%$ CI $0.97-0.99)$.

Discussion: Age, gender and BMI were key predictors of FF. General Practitioners appeared to be monitoring vitamin $\mathrm{D}$, with extensive usage of vitamin $\mathrm{D}$ and calcium. However, they less commonly give osteoporosis specific health, dietary or exercise advice, or at least are not coding having done so using osteoporosis specific codes.

Conclusion: Falls, FF and osteoporosis were common in South Asian adults over the age of 50. Primary Care physicians are commonly monitoring vitamin $\mathrm{D}$ and also prescribing vitamin $\mathrm{D}$ and calcium supplements but less attention may be being paid to osteoporosis specific health, diet and exercise advice.

This research was funded by the Royal Osteoporosis Society.

\section{Abstract ID: 22 \\ Original Research \\ Subcategory: Fracture risk}

\section{Social isolation in older adults and implications for bone health: findings from the Hertfordshire cohort study}

G Bevilacqua', KA Jameson', J Zhang', MA Clynes' ${ }^{1}$ C Cooper ${ }^{1,2}$, EM Dennison ${ }^{1,3}$

'MRC Lifecourse Epidemiology Unit, University of Southampton, Southampton, UK, ${ }^{2}$ NIHR Musculoskeletal Biomedical Research Unit, University of Oxford, Oxford, UK, ${ }^{3}$ School of Biological Sciences, Victoria University of Wellington, Wellington, New Zealand

Introduction: Previous studies have found that social isolation is prevalent in the elderly and that the number of older adults at risk of becoming isolated is increasing. Social isolation has been associated with lifestyle factors linked to poorer musculoskeletal health. We therefore sought to study associations between social isolation and bone health in older communitydwelling adults.

Methods: Hertfordshire Cohort Study participants [222 men and 221 women; mean (SD) age 75.5 (2.5) years for men and 75.8 (2.6) for women] were visited at home by a trained fieldworker, who administered a lifestyle questionnaire that included the Hospital Anxiety and 
Depression (HAD) Scale. Participants attended a research clinic for bone mineral density (BMD) assessment using a dual X-ray absorptiometry (DXA) scan (Lunar Prodigy). Social isolation was assessed using the six-item Lubben Social Network Scale (LSNS-6) and the Maastricht Social Participation Profile (MSPP).

Results: Data were available for 369 participants (185 men and 184 women). Social isolation was high in our sample of community-dwelling adults: $40 \%$ of men and $42.4 \%$ of women were identified as socially isolated on either the LSNS-6 or MSPP scales. Seven percent of men and $9.8 \%$ of women reported having previously been diagnosed with depression by a doctor. Isolated men and women had a higher odds of depressive disorder (HAD-D); in men, the OR was $4.84,95 \%$ CI $0.97-24.15, p=0.05$, and in women OR was 3.03, 95\% CI 0.93-9.86, $p=0.07$ for depression in individuals who reported isolation compared to those who were non-isolated (results adjusted for the following confounders: age, BMI, smoker status, alcohols consumption,

Table 1. Comparison of patients with and without liver cirrhosis

\begin{tabular}{|c|c|c|c|}
\hline & Controls & Liver cirrhosis Cases & $\mathrm{P}$ value \\
\hline Male & $19(63 \%)$ & $19(63 \%)$ & 1 \\
\hline Age (years) & $65.5 \pm 11$ & $63.8 \pm 10.8$ & 0.55 \\
\hline Body mass index (BMI) & $27.9 \pm 4.3$ & $29.5 \pm 4.5$ & 0.16 \\
\hline Femoral neck T-score & $-1.36 \pm 1.24$ & $-1.17 \pm 1.14$ & 0.53 \\
\hline Total hip T-score & $-0.78 \pm 1.19$ & $-0.56 \pm 1.2$ & 0.48 \\
\hline Lumbar spine T-score & $-0.78 \pm 1.80$ & $-0.60 \pm 1.82$ & 0.69 \\
\hline Lumbar spine BMD $\left(\mathrm{g} / \mathrm{cm}^{2}\right)$ & $0.989 \pm 0.20$ & $1.001 \pm 0.21$ & 0.83 \\
\hline Lumbar spine TBS (unitless) & $1.35 \pm 0.14$ & $1.20 \pm 0.17$ & $<0.001^{*}$ \\
\hline TBS T-score & $-1.02 \pm 1.27$ & $-2.3 \pm 1.62$ & $<0.001^{*}$ \\
\hline Degraded TBS (TBS T-score $\leq-2.5$ ) & $10 \%$ & $40 \%$ & $<0.001^{*}$ \\
\hline Osteoporosis (lowest BMD T-score $\leq-2.5$ ) & $26.6 \%$ & $30.4 \%$ & 0.77 \\
\hline FRAX major osteoporotic fracture (\%) & $10.64 \pm 8.8$ & $8.15 \pm 8.34$ & 0.38 \\
\hline FRAX hip fracture (\%) & $3.39 \pm 4.25$ & $2.26 \pm 4.33$ & 0.42 \\
\hline Current smoking & $13 \%$ & $26 \%$ & 0.23 \\
\hline Alcohol excess ( $>3$ units daily) & $4.7 \%$ & $6.6 \%$ & 0.87 \\
\hline
\end{tabular}
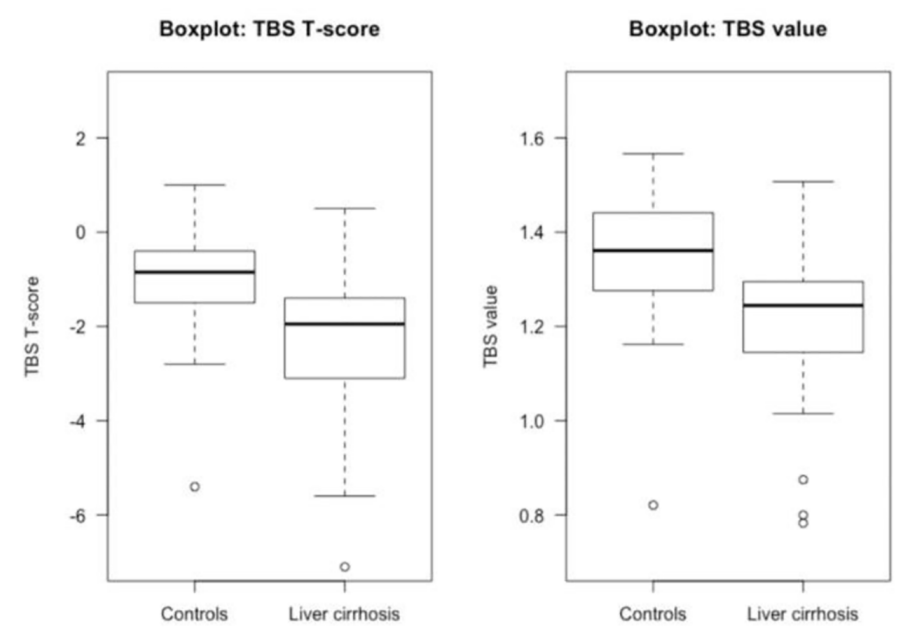

social class, physical activity time, and prudent diet score). There were however no associations between social isolation and BMD in this study.

Conclusion: We report high levels of social isolation in community-dwelling older adults; this was associated with an increased risk of depression, which may lead to poorer bone health in the future. Longitudinal studies are warranted.

\section{Abstract ID: 23 \\ Original Research \\ Subcategory: DXA}

\section{Trabecular bone score as a risk factor of bone disease in liver cirrhosis}

\section{CR Rakieh ${ }^{1}$, SS Silva', TR Roberts', DEP \\ Powell', SH Ho ${ }^{2}, \mathrm{RB}$ Butler ${ }^{1}$}

${ }^{1}$ Rheumatology, Robert Jones and Agnes Hunt Orthopaedic Hospital, Oswestry, UK, ${ }^{2}$ Geriatric Medicine, Robert Jones and Agnes Hunt Orthopaedic Hospital, Oswestry, UK

Background: Trabecular bone score (TBS) is an index of bone microarchitecture and has been validated as an independent risk factor for fracture. Reduced lumbar spine (LS) TBS has been found to be associated with secondary osteoporosis, but no studies have been published to date on TBS in patients with liver cirrhosis.

Objective: To compare LS TBS in subjects with and without liver cirrhosis and assess whether TBS can be used as a bone risk factor independent of bone mineral density (BMD).

Methods: Case control study. Patients $\geqslant 18$ years with body mass index (BMI) of 15-37 were eligible. Dual energy x-ray absorptiometry (DXA) BMD and LS TBS were measured in 30 consecutive patients with liver cirrhosis and 30 controls matched for sex, age ( \pm 3 years), BMI $\left( \pm 3 \mathrm{~kg} / \mathrm{m}^{2}\right)$, and lumbar spine BMD $( \pm 0.03 \mathrm{~g} /$ $\mathrm{cm}^{2}$ ). Degraded TBS was defined as TBS $\mathrm{T}$-score $\leqslant-2.5$.

Results: $63 \%$ of the patients were males. TBS measurements were significantly lower in the liver cirrhosis group compared to the matched control group $(p<0.001) .40 \%$ of those with cirrhosis had degraded TBS compared to $10 \%$ of the controls. The risk of having degraded TBS was significantly higher in cirrhotic patients compared to controls; odds ratio $=6(95 \%$ confidence interval $=1.48-24.3$ ). 
Discussion: Degraded TBS is common in liver cirrhosis. Cirrhotic patients had lower TBS than controls matched for spine BMD and other potential confounders (such as age and BMI) demonstrating bone microarchitecture deterioration as an independent marker in these patients.

Conclusion: Compromised bone quality may have an important role in the aetiology of metabolic bone disorder associated with chronic liver disease. TBS should be considered as a risk factor of bone disease in these patients. Other studies are needed to validate these results and assess the prognostic value of TBS in liver disease.

\section{Abstract ID: 25 \\ Original Research \\ Subcategory: Vit D}

\section{Circulating vitamin $\mathrm{D}$ and bone health in midlife: findings from the Hertfordshire cohort study}

\section{FL Laskou', GB Bevilacqua', MC Clynes', KJ Jameson', BB Boucher², KN Noonan², CC Cooper ${ }^{1}$, ED Dennison ${ }^{1}$ \\ 1MRC Lifecourse Epidemiology Unit, University of Southampton, Southampton, UK, ${ }^{2}$ The Blizard Institute, Bart's \& The London School of Medicine and Dentistry, Queen Mary University of London, London, UK, ${ }^{3}$ Department of Clinical Biochemistry, Barts NHS Trust, London, UK}

Objective: Vitamin D insufficiency is common in later life, and is often reported in older institutionalised adults, but fewer data are available in midlife. Low circulating $25(\mathrm{OH})$ vitamin D [25( $\mathrm{OH}) \mathrm{D}]$ levels has been linked to low bone mineral density (BMD) and increased fracture rates. Most studies, though, have reported findings in institutionalised older cohorts with severe vitamin $\mathrm{D}$ deficiency. In this study we report the association of circulating concentration of serum $25(\mathrm{OH}) \mathrm{D}$ in a community cohort of adults in midlife and BMD.

Methods: Participants were recruited from the Hertfordshire Cohort Study, an established longitudinal study of community-dwelling adults. Lumbar spine and total femur BMD were measured using a Hologic QDR 4500 instrument. Serum 25(OH)D concentrations were measured using a DiaSorin Liason chemiluminescent assay.

Results: 820 subjects (397 men, 423 women) participated. The median (IQR) age of participants was $64.0(61.8-66.5)$ and $65.5(63.3-67.6)$ years for men and women respectively. Median circulating concentration of $25(\mathrm{OH}) \mathrm{D}$ was $44.6(35.0-$ 63.0) $\mathrm{nmol} / 1$ and $41.3(29.8-53.5) \mathrm{nmol} / \mathrm{l}$ in men and women respectively. Circulating $25(\mathrm{OH}) \mathrm{D}$ values were associated with season of blood testing $(p=0.001)$ and with supplement use $(p=0.002)$. Higher femoral neck BMD was observed in men, but not women, with higher vitamin $\mathrm{D}$ status after adjustment for age, season, BMI, smoker status, alcohol consumption, physical activity and social class $(p=0.01)$.

Conclusion: Mild vitamin D insufficiency is common in community-dwelling adults in midlife, and seasonal fluctuations in its levels were also observed as expected. Circulating $25(\mathrm{OH}) \mathrm{D}$ concentration was associated with hip BMD in men, highlighting the need to promote an adequate vitamin $\mathrm{D}$ intake over the winter months in men, as well as in women.

\section{Abstract ID: 27 \\ Original Research \\ Subcategory: DXA}

\section{Association between bone mineral density and arteriosclerosis in postmenopausal women: a 10-year follow- up of the Japanese population-based osteoporosis cohort study}

M Jaalkhorol' ${ }^{1}$, F Fujita ${ }^{2}$, M Iki², K Kouda'2, A Yura ${ }^{2}, J$ Tamaki ${ }^{3}$, M Komatsu', D Namiraa ${ }^{5}$, E Kajita' ${ }^{6}$, T Tachiki', S Kagamimori ${ }^{7}$

1 Preventive Medicine, Mongolian National University of Medical Sciences, Ulaanbaatar, Mongolia, ${ }^{2}$ Public Health, Kindai University Faculty of Medicine, Osaka, Japan, ${ }^{3}$ Hygiene \& Public Health, Osaka Medical College,

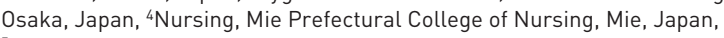
${ }^{5}$ Orthopedic Medicine, Second Affiliated Hospital of Inner Mongolia Medical University, Huhhot, China, ${ }^{6}$ Public Health and Home Nursing, Nagoya University, School of Health Sciences, Nagoya, Japan, ${ }^{7}$ University of Toyama, 7 University of Toyama, Toyama, Japan

Background: Arteriosclerosis and osteoporosis may progress in common pathological conditions such as oxidative stress and glycoxidative damage. Previous studies show that arteriosclerosis increases the risk of osteoporosis. However, little is known whether low bone mineral density (BMD) predicts the incidence of arteriosclerosis.

Objective: This study assessed the association between arteriosclerosis and BMD in the 10-year follow-up of the Japanese Population-Based Osteoporosis (JPOS) cohort study. 
Methods: A total of 725 women aged $\geqslant 50$ years participated in the 2006 survey (baseline). Arteriosclerosis was defined as value of brachialankle pulse wave velocity $(P W V) \geqslant 1800 \mathrm{~cm} / \mathrm{s}$. We analyzed data from 446 women who completed measurements of brachial-ankle pulse wave velocity (PWV) at both baseline and followup surveys (in 2012 and/or 2015), after excluding 191 women with $\mathrm{PWV} \geqslant 1800 \mathrm{~cm} / \mathrm{s}$ at baseline. $\mathrm{BMD}$ at the lumbar spine, total hip, and femoral neck were measured by dual-energy X-ray absorptiometry at baseline.

Results: We identified 166 women with PWV $\geqslant 1800 \mathrm{~cm} / \mathrm{s}$ in the follow-up surveys. Odds ratios (OR) for a $1 \mathrm{SD}$ decrease in $\mathrm{BMD}$ were significant at the total hip [OR $=1.5,95 \%$ confidence interval $(\mathrm{Cl}): 1.03-1.64]$ and femoral neck $(\mathrm{OR}=1.3$, 95\% Cl: 1.03-1.64) after adjusting for age, BMI, smoking, diabetes mellitus, and hyperlipidemia.

Conclusions: Our findings suggest that low BMD may predict the subsequent progress of arteriosclerosis.

\section{Abstract ID: 28 \\ Original Research \\ Subcategory: Fracture risk}

\section{Supporting teams to prevent secondary fractures in patients over 50 using a breakthrough series collaborative approach}

G Rice1, B Wiles', A Cracknell1,3, C Spice ${ }^{1,4}$, MK Javaid ${ }^{1,4}$

${ }^{1}$ Care Quality Improvement Department, Royal College of Physicians, London, UK, ${ }^{2}$ Department of Medicine for Older People, Leeds Teaching Hospitals NHS Trust, Leeds, UK, ${ }^{3}$ Department of Older Persons Medicine, Portsmouth Hospitals NHS Trust, Portsmouth, UK, ${ }^{4}$ Nuffield Department of Orthopaedics, Rheumatology and Musculoskeletal Sciences, University of Oxford, Oxford, UK

Aims: The patient, clinical and health-economic benefits from secondary fracture prevention are accepted, and both national and international guidelines recommend the Fracture Liaison Services (FLS) model. The national Fracture Liaison Service Database (FLSDB) audit provides real-time performance metrics at the FLS and national level. Over the years improvements have been made in FLS provision within England and Wales but gaps remain. The Royal College of
Physicians' Quality Improvement (QI) programme supports the establishment of QI in clinical settings. We aimed to test the impact of adding a 'Breakthrough Series Collaborative' on delivery of clinical outcomes.

Methods: Using the Institute for Healthcare Improvement's 'Breakthrough Series Collaborative ${ }^{1}$ methodology, an FLS specific curriculum was developed. The curriculum included the theory of the Model for Improvement, examples specific to the FLS setting and outcome metrics from the FLSDB. The content was delivered to ten FLSs from across the UK, in three face-to-face meetings, providing valuable time away from the clinical setting to learn more about QI, supporting peer-to-peer exchanges of ideas and learning. Between learning sessions, teams were supported by an expert QI faculty and the FLSDB team through coaching calls.

Results: The ten FLSs identified three themes for improvement: identification of vertebral fractures; post-fracture follow up and monitoring; identification and bone health management of inpatients with previous fractures. All ten FLSs were able to setup a local QI team that met regularly, design sustainable interventions and deliver at least one cycle of plan-do-study-act. Nine FLSs were actively participating in the FLSDB and $40 \%$ were able to demonstrate a statistically significant improvement on their initial data that had not been present in the past 2 years of FLSDB participation. Comparing pre with post-collaborative surveys, there was improved confidence in QI principles, using data for improvement and a team approach to overcoming challenges.

These findings demonstrate the benefit of active involvement of FLSs in QI to maximise patient outcomes from FLS' participation with national audits. Further work is needed to quantify the scale of patient benefit and its sustainability to justify specific inclusion of time for QI in the commissioning of FLSs in the NHS.

\section{References}

1. The breakthrough series: IHI's collaborative model for achieving breakthrough improvement. IHI Innovation Series white paper. Boston: Institute for Healthcare Improvement, www.IHI.org. (2003). 


\section{Abstract ID: 29 \\ Original Research \\ Subcategory: Management and treatment}

\section{Is there an endogenous circadian rhythm in bone turnover?}

\section{AL Darling', F Gossiel'2, BA Middleton³, $R$ Eastell'2, SA Lanham-New', DJ Skene ${ }^{3}$}

'Department of Nutrition, Food and Exercise Sciences, University of Surrey, Guildford, UK, ${ }^{2}$ The Mellanby Centre for Bone Research, University of Sheffield, Sheffield, UK, ${ }^{3}$ Section of Chronobiology, University of Surrey, Guildford, UK

Background: Previous studies have attempted to demonstrate a circadian rhythm in bone turnover but have only shown diurnal $24 \mathrm{~h}$ rhythms, due to not having controlled the possible confounding effects of meals, posture, activity, sleep/ wake and light/dark conditions.

Objective: To assess whether bone turnover in humans has a true circadian rhythm using the gold-standard constant routine protocol to remove/minimise confounding effects.

Methods: Serum procollagen type I N-terminal propeptide (PINP) and C-terminal telopeptide of type I collagen (CTX) was measured in banked 2 hourly samples taken sequentially across 26 hours. These samples were from a previously conducted constant routine study $(n=22$ individuals ( $n=11$ females), aged 19-27years). All females were taking combined oral contraceptives. Measurements of bone markers were undertaken using automated electro-chemiluminescent immunoassay (ECLIA) (Cobas e411, Roche Diagnostics). To assess circadian rhythmicity cosinor/linear analysis was undertaken on Z-scored data using MATLAB 2019b software.

Results: In men, 9/11 (82\%) had a statistically significant $(p<0.05$, rhythmicity $\geqslant 40 \%)$ cosine fit for CTX, with a mean ( \pm SEM) acrophase time (peak) at 02:58h ( $\pm 00: 49)$ and an amplitude of $0.17(0.1) \mathrm{ng} / \mathrm{mL}$. All the females had a statistically significant cosine + linear fit for CTX, with an acrophase time of 03:24h ( $\pm 00: 18)$ and an amplitude of $0.05(0.01) \mathrm{ng} / \mathrm{mL}$. By contrast, only $27 \%$ of both males and females showed significant PINP rhythms (acrophase and amplitude thus not calculated).

Discussion: Serum CTX, but not PINP, exhibits a clear circadian rhythm in both males and females. Sex differences in the shape of the CTX rhythm may be the result of the daily oral dose of oestrogen in the oral contraceptives.

Conclusion: Our analysis is the first to demonstrate a true endogenous circadian rhythm in serum CTX in men and women. It will now be of importance to investigate whether this rhythm is disrupted in osteoporosis and other bone disorders.

This work was funded by a Royal Osteoporosis Society Project Grant (Grant No. 420).

\section{Abstract ID: 30 \\ Original Research \\ Subcategory: Management and treatment}

\section{*Effect of selenium supplementation on biomarkers of bone turnover}

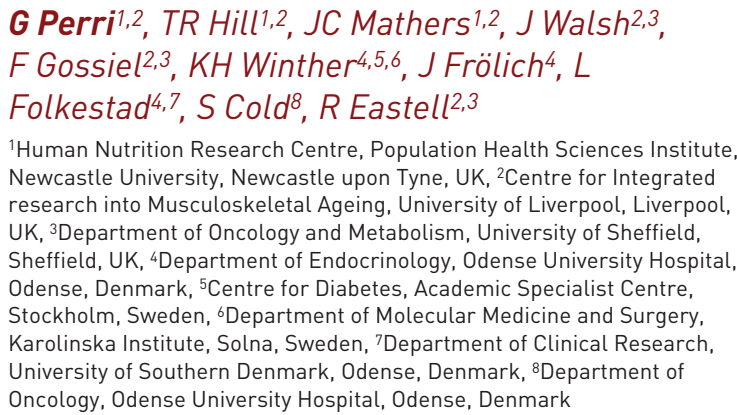

${ }^{1}$ Human Nutrition Research Centre, Population Health Sciences Institute, Newcastle University, Newcastle upon Tyne, UK, ${ }^{2}$ Centre for Integrated research into Musculoskeletal Ageing. University of Liverpool, Liverpool, UK, ${ }^{3}$ Department of Oncology and Metabolism, University of Sheffield, Sheffield, UK, 4Department of Endocrinology, Odense University Hospital, Odense, Denmark, ${ }^{5}$ Centre for Diabetes, Academic Specialist Centre, Stockholm, Sweden, 'Department of Molecular Medicine and Surgery, Karolinska Institute, Solna, Sweden, ${ }^{7}$ Department of Clinical Research, University of Southern Denmark, Odense, Denmark, ${ }^{8}$ Department of Oncology, Odense University Hospital, Odense, Denmark

Background: Selenium is an essential trace element for human health. Osteoclast inactivation is associated with selenium supplementation in vitro and selenium status is correlated negatively with markers of bone turnover (BTM). However, the impact of selenium supplementation on BTM has not been studied.

Objective: To investigate the effects of selenium supplementation on BTM including OC, P1NP, CTX and BAP in the short- and long-term.

Methods: 491 Danish men and women (6074 year) were randomised to receive $0,100,200$ or $300 \mu \mathrm{g}$ of selenium-enriched yeast daily. Serum selenium concentration was measured using inductively-coupled-plasma mass spectrometry and BTM were measured using an autoanalyser (IDS iSYS) at baseline, 6 months and 5 years in non-fasted samples. Data were analysed by ANCOVA with polynomial contrasts to investigate the shape of the dose-response relationships. Covariates included: age, BMI, baseline selenium 


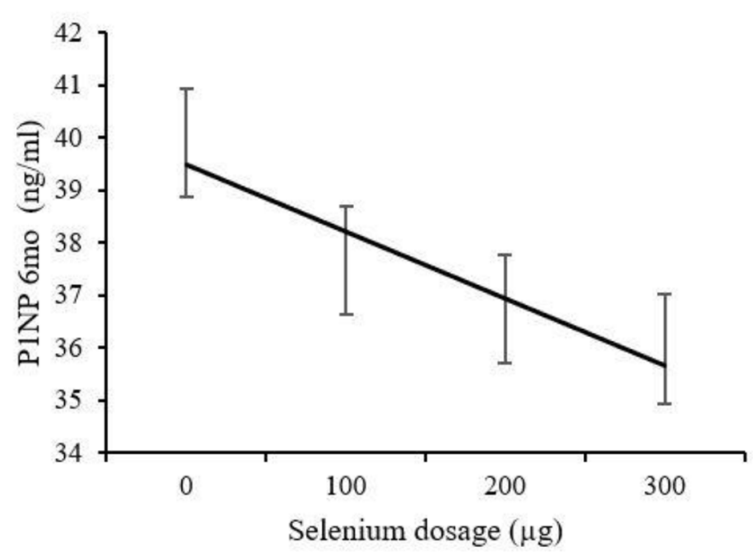

Figure 1. Mean concentration of serum PINP at 6 months following selenium supplementation at does: 0, 100, 200 and $300 \mu \mathrm{g}$. Points are means from the ANCOVA with a fitted linear regression line. SE bars are displayed.

status, baseline BTM, smoking, alcohol, supplement use and medication.

Results: Plasma selenium concentration increased significantly with increasing selenium supplementation at 6 months and remained elevated at 5 years $(p<0.001)$. Selenium status remained constant in the placebo-treated group over time. At 6 months serum selenium concentration increased by 79,145 and $203 \%$ for doses $100-300 \mu \mathrm{g}$, respectively. At 6 months, there was a significant linear decrease in P1NP $(p=0.019)$ with increasing selenium supplementation (Figure 1). There was no significant effect of selenium supplementation on any other BTM.

Discussion: P1NP is a marker of osteoblast function. The fall in PINP with increasing selenium intake suggests a reduction in new bone formation.

Conclusions: Selenium supplementation reduced P1NP at 6 months but there were no significant effects on other BTM or at later times. The impact of this change in bone turnover on bone health remains to be determined.

\section{Abstract ID: 32 \\ Original Research \\ Subcategory: Management and treatment \\ *Self-perception of fracture risk and use of anti-osteoporosis medication among}

participants in the GLOW study: a possible mechanism to boost medication adherence?

J Zhang 1, S Carter1, A Litwic 1, LD Westbury',
K Ward1 , C Cooper1,2, EM Dennison'1,3
'MRC LEU, MRC LEU, Southampton, UK, 2NIHR Musculoskeletal
Biomedical Research Unit, University of Oxford, Oxford, UK, 3Victoria
University of Wellington, Victoria University of Wellington, Wellington, UK

Objective: Self-perception of fracture risk (SPR) asks individuals to rate their risk of fracture relative to others of the same age and has been associated with fracture risk independent of FRAX score. Here we considered how SPR relates to the use of anti-osteoporosis medication (AOM) with regard to initiation and continuation in women over the age of 55 years.

Methods: GLOW is a global longitudinal study of the bone health of 60,000 female participants, aged 55 or older, including the UK. At baseline, and at 1-, 2- and 3-year follow-ups, participants completed questionnaires regarding their medical history, current medication, and SPR. Among 3912 participants with data on baseline SPR and AOM at one or more time-points, the association between baseline SPR and use of AOM at each time-point was examined using logistic regression. In a subsample of 331 participants taking AOM at baseline, SPR at baseline in relation to continued use of AOM up to the 3-year follow-up was also examined.

Results: The mean baseline age of participants was 69 (SD 9) years. Higher SPR at baseline was significantly positively associated with taking $\mathrm{AOM}$ at baseline (odds ratio per higher band of SPR: 3.47, CI 95\% 3.00, 4.02, $p<0.001)$ and at each follow-up (Year 3: 2.50, CI 95\% 2.16, 2.89, $p<0.001)$; relationships were robust before and after adjustment for baseline FRAX score. Among the subsample taking AOM at baseline, participants with higher SPR at baseline were significantly more likely to have continued taking AOM over the 3-year follow-up period (odds ratio per higher band: 1.33 , CI 95\% 1.06, 1.66, $p=0.013$ ) than those with lower baseline SPR.

Conclusions: Perhaps unsurprisingly, SPR is associated with baseline use of AOM in this group, with associations robust to adjustment for baseline FRAX score. Importantly however, GLOW participants with higher SPR were more likely to persist with AOM over the 3-year follow-up, suggesting that education involving SPR might be a strategy to boost long-term AOM adherence. 


\section{Abstract ID: 34 \\ Original Research}

The efficacy of adding a personalised exercise programme to improve strength and balance in individuals following a fragility fracture: a single cohort study

\section{AK Sularz', G Darnell2, M Vindlacheruvu ${ }^{3}$}

'School of Clinical Medicine, Cambridge University Hospitals NHS Foundation Trust, Cambridge, UK, ${ }^{2}$ Department of Physiotherapy, Cambridge University Hospitals NHS Foundation Trust, Cambridge, UK, ${ }^{3}$ Department of Geriatric Medicine, Cambridge University Hospitals NHS Foundation Trust, Cambridge, UK

Background: Physiotherapy and exercise are valuable components of our commissioned Fracture Liaison Service (FLS). We provide vulnerable fragility fracture patients with Individualised Exercise Programmes (IEP) as per evidence-based recommendations. ${ }^{1}$
Objective: To evaluate physiotherapy-led IEPs as a means of reducing the risk of falls in a highrisk cohort.

Methods: Female patients $(n=35$; mean age 76.5) with falls risk factors were recruited from FLS clinic (Table 1). Inclusion criteria included (i) previously independent community dwelling older adults, and (ii) cognitively able to follow instructions. Outcome measures used were the Timed Up and Go Test (TUG) ${ }^{2}$ and the 30 Second Chair Stand Test (30CST). ${ }^{3}$ A one-way repeated measures analysis of variance (ANOVA) was used to analyse outcomes before and after 5 weeks training, and at 3 month follow-up. A separate paired $t$-test was conducted for patients who were lost to follow-up to minimise selection bias.

Table 1. Study cohort details. Patients 1-13 were present at three-month follow-up. All patients were female (NA not available; IEP individualised exercise program; NOF neck of femur; PT physiotherapy).

\begin{tabular}{|c|c|c|c|c|c|c|c|c|c|}
\hline \multirow[t]{2}{*}{ Patient } & \multirow[t]{2}{*}{ Age } & \multirow[t]{2}{*}{ Fracture } & \multirow[t]{2}{*}{$\begin{array}{l}\text { Previous } \\
\text { Fractures }\end{array}$} & \multicolumn{3}{|c|}{ T-Score } & \multirow[t]{2}{*}{$\begin{array}{l}\text { Physiotherapy } \\
\text { sessions } \\
\text { completed }\end{array}$} & \multirow[t]{2}{*}{$\begin{array}{l}\text { Recommendations } \\
\text { on discharge }\end{array}$} & \multirow[t]{2}{*}{ Additional activity } \\
\hline & & & & Spine & Hip & NOF & & & \\
\hline 1 & 76 & Left distal radius & 0 & -2.3 & -2.1 & -2.5 & 5 & IEP & \\
\hline 2 & 61 & Left distal radius & 0 & -3.0 & -1.3 & -0.6 & 5 & IEP & \\
\hline 3 & 53 & Left tibial plateau & 0 & -2.6 & -1.2 & -2.1 & 5 & IEP & Gym \\
\hline 4 & 59 & Right ankle & 0 & -2.9 & -1.3 & -1.4 & 5 & IEP & \\
\hline 5 & 69 & Left distal radius & 0 & -2.5 & -1.8 & -2.2 & 5 & IEP & Local exercise group \\
\hline 6 & 59 & Left humerus & 0 & -2.8 & -1.6 & -1.4 & 5 & IEP & \\
\hline 7 & 63 & Left glenoid & 0 & -3.9 & -2.5 & -2.5 & 4 & IEP & \\
\hline 8 & 61 & Left ankle & 1 & -2.5 & -1.3 & -1.3 & 5 & IEP & Rowing + weight club \\
\hline 9 & 73 & Left hallux & 0 & -3.9 & -2.6 & -2.6 & 5 & IEP & \\
\hline 10 & 64 & Right distal radius & 0 & -3.3 & 0.3 & -0.8 & 4 & IEP & \\
\hline 11 & 76 & Left humerus & 3 & -2.7 & -2.1 & -2.5 & 5 & IEP & \\
\hline 12 & 58 & Left wrist & 0 & -3.0 & -1.6 & -2.0 & 5 & IEP & Running \\
\hline 13 & 74 & Left 5th metacarpal & 2 & -3.4 & -1.5 & -2.1 & 5 & IEP & \\
\hline 14 & 86 & Left patella & 4 & NA & NA & NA & 4 & IEP & Everyone Health Falls \\
\hline 15 & 63 & Right hip & 1 & NA & NA & NA & 5 & IEP & Local exercise group \\
\hline 16 & 54 & Right distal radius & 0 & -2.4 & 0.1 & 0.7 & 5 & IEP & Gym \\
\hline 17 & 82 & Right hip & 0 & NA & NA & NA & 5 & IEP & \\
\hline 18 & 76 & Left hip & 0 & NA & NA & NA & 4 & IEP & Walking group \\
\hline 19 & 70 & Right ankle & 0 & -3.5 & -2.6 & -2.8 & 5 & IEP & \\
\hline 20 & 91 & Right wrist & NA & NA & NA & NA & 5 & IEP + community PT & \\
\hline 21 & 55 & Left wrist & 0 & -3.5 & -1.4 & -2.5 & 5 & IEP + exercise referral & \\
\hline 22 & 56 & Left ankle & 3 & -3.7 & -2.0 & -1.8 & 4 & IEP + exercise referral & \\
\hline 23 & 74 & Left and right wrists & 0 & -1.8 & -0.5 & -1.5 & 5 & IEP & \\
\hline 24 & 71 & Right hip & 0 & -0.9 & -2.6 & -1.0 & 4 & IEP & \\
\hline 25 & 82 & Right hip & 0 & NA & NA & NA & 4 & IEP + community PT & \\
\hline 26 & 73 & Right hip & 0 & -2.8 & -2.3 & -2.4 & 5 & Exercise referral & \\
\hline 27 & 73 & Left hip & 1 & -3.6 & -2.4 & -1.7 & 5 & Exercise referral & \\
\hline 28 & 65 & Left tibial plateau & 1 & -1.8 & -1.8 & -1.7 & 5 & IEP & \\
\hline 29 & 72 & Right humerus & 0 & -1.5 & -0.5 & -1.1 & 5 & IEP + exercise referral & \\
\hline 30 & 87 & Left fibula & 0 & NA & NA & NA & 5 & Exercise referral & \\
\hline 31 & 68 & Right radius & 0 & -1.1 & -1.3 & -0.5 & 3 & IEP & \\
\hline 32 & 63 & Left olecranon & 0 & -2.6 & -1.6 & -2.6 & 5 & IEP & Gym $+50+$ classes \\
\hline 33 & 60 & Left radius & 0 & -1.7 & -1.8 & -1.6 & 4 & IEP & \\
\hline 34 & 59 & Left radius & 0 & -3.1 & -2.0 & -2.6 & 5 & Exercise referral & \\
\hline 35 & 77 & Left hip & 0 & -1.2 & -2.1 & -2.6 & 4 & IEP & Gym \\
\hline
\end{tabular}


Results: Thirty-five patients were tested before and after the training. Only thirteen were present at follow-up due to the COVID-19 pandemic. ANOVA revealed a statistically significant effect of training for TUG $(\mathrm{F}(2,24)=23.994, p=0.001)$ and 30CST $(\mathrm{F}(2,24)=68.527, p=0.001)$. Paired $t$-test analysis on the attrition group was statistically significant for both TUG [pre-training $(M=9.536, S D=3.578)$, immediate post-training $(\mathrm{M}=9.002, \mathrm{SD}=3.526): \mathrm{t}(21)=14.129$, $p=0.001)$ ] and 30CST [pre-training $(M=12.273$, $\mathrm{SD}=5.548)$, immediate post-training $(M=14.273$, $\mathrm{SD}=5.824): \mathrm{t}(21)=-7.165, p=0.001)]$.

Discussion: The outcome measures were validated, reliable and feasible. Further studies are required to determine intervention generalisability.

Conclusion: Physiotherapy-led exercise significantly improves TUG and 30CST scores in highly motivated fragility fracture patients, with sustained improvement at three-month follow-up.

\section{References}

1. Royal Osteoporosis Society. Strong, steady and straight. Royal Osteoporosis Society, theros.org. uk/forms/documents/strong-steady-and-straight (2018)

2. Shumway-Cook A, Braver S and Woollacott M. Predicting the probability of falls in communitydwelling older adults using the timed up and go test. Phys Therapy 2000; 80: 896-903.

3. Jones CJ, Rikli RE and Beam WC. A 30-s chairstand test as a measure of lower body strength in community-residing older adults. Res $Q$ Exerc Sport 1999; 70: 113-119.

\section{Abstract ID: 36 \\ Original Research \\ Subcategory: Management and treatment \\ Associations of polyunsaturated fatty acids with markers of bone turnover in post- menopausal women}

\section{o Feehan', DJ Armstrong², MM Slevin', PJ Magee', LK Pourshahidi', PJ Allsopp', EM McSorley ${ }^{1}$}

${ }^{1}$ Nutrition Innovation Centre for Food and Health (NICHE), Ulster University, Coleraine, UK, ${ }^{2}$ Fracture Liaison and Osteoporosis
Service, Altnagelvin Hospital, Western Health and Social Care Trust, Londonderry, UK

The immunomodulatory properties of n-3 polyunsaturated fatty acids (PUFA) are purported to favourably alter bone remodelling through a reduction of bone resorption that is known to contribute to age associated bone loss in postmenopausal populations.

This study investigated the associations between serum PUFA and bone turnover markers (BTMs) in 300 non-osteoporotic post-menopausal women. Serum BTMs: osteocalcin, C-terminal telopeptides of type 1 collagen (CTX) total alkaline phosphatase, and urinary BTMs: creatinine corrected urinary deoxypyridinoline $(\mathrm{DPD} / \mathrm{Cr})$ and CTX/Cr, were quantified. Total n-6 PUFA (linoleic acid; LA \& arachidonic acid; AA) and n-3 PUFA ( $\alpha$-linolenic acid; ALA + eicosapentaenoic acid; EPA + docosapentaenoic acid; DPA + docosahexaenoic acid; DHA) were measured. Total n-6:n-3 ratio and LA:ALA ratios were calculated. The mean $\pm S D$ age of this cohort was $61 \pm 6.4$ years with a mean body mass index (BMI) of $27.4 \pm 4.8 \mathrm{~kg} / \mathrm{m}^{2}$ and were on average $12.6 \pm 7.6$ years post-menopause. Spearman correlations identified a significant negative correlation between the resorption marker urinary DPD/ Cr and total n-3 PUFA $(r=-0.137, p=0.018)$ as well as EPA $(r=-0.150, p=0.009)$ and DPA $(r=-0.118, p=0.042)$. A significant negative correlation was observed between the resorption marker urinary $\mathrm{CTX} / \mathrm{Cr}$ and AA $(r=-0.121$, $p=0.036)$ and ALA $(r=-0.123, p=0.033)$. A significant positive correlation was found between urinary CTX/Cr and the LA:ALA ratio $(r=0.145$, $p=0.012$ ). Multiple regression identified a significant negative association between ALA and urinary $\mathrm{DPD} / \mathrm{Cr}(\beta=-0.141, p=0.016)$ independent of age, BMI, physical activity, smoking status and years post-menopause. In similar regression models, the LA:ALA ratio was positively associated with urinary $\mathrm{DPD} / \mathrm{Cr}(\beta=0.137$, $p=0.019$ ).

In summary, serum n-3 PUFA status was associated with lower bone resorption whereas a higher serum $n-6: n-3$ ratio was associated with greater bone resorption highlighting a possible beneficial role for dietary intake of n-3 PUFA in reducing post-menopausal bone loss. 


\section{Abstract ID: 37 \\ Original Research \\ Subcategory: Patient experience \\ Quality and readability of online \\ osteoporosis patient information resources}

F Crawford-Manning ${ }^{1,2}$, C Greenall ${ }^{2}$,

L Bullock², A Hawarden², S Leyland ${ }^{3}$, C Jinks²,

J Protheroe2, Z Paskins ${ }^{1,2}$

${ }^{1}$ Osteoporosis Service, Midlands Partnership NHS Foundation Trust, Haywood Hospital, Stoke-on-Trent, UK, ${ }^{2}$ School of Primary, Community and Social Care, Keele University, Staffordshire, UK, ${ }^{3}$ Royal Osteoporosis Society, Bath, UK

Background: The internet is increasingly becoming an influential source of health care information. Although the internet provides a platform for patient engagement with their health conditions, the quality of information is not guaranteed. The quality of patient information encompasses a range of issues including accessibility, content (health literacy), acceptability and presentation style.

Objective: To identify common patient information resources about osteoporosis and osteoporosis drug treatment and appraise the readability and quality.

Methods: Patient information resources were purposively sampled and assessed for readability (Flesch Reading Ease (FRE) and Flesch-Kincaid Grade Level (FKGL)). Acceptable readability was defined as FRE $>60$, FKGL $<6$ (reading age of approximately 11), in accordance with the recommended readability level for provision of medical information. The overall quality of each resource was also assessed utilising a modified International Patient Decision Aid Standard (m-IPDAS). The m-IPDAS assess patient information based on 32 items across 8 categories of: content, bias \& detail, probabilities, accuracy, decision making, conflict of interest, structure \& layout, and reliability.

Results: Nine patient information websites and leaflets were selected, from government, charity and private healthcare providers. $8 / 9$ of the selected resources had an FRE readability score below acceptable, whereas all resources had acceptable FKGL readability scores (FRE 48.262; FKGL 8.0-12.8). Quality scores from the m-IPDAS were all $<70 \% \quad(21.9-65.6 \%)$. Resources scored poorly in the m-IPDAS domains of 'accuracy' and 'probabilities', meaning many resources failed to provide reliable evidence, or present the chance of osteoporosis and/or osteoporosis drug treatment outcomes in an understandable way. Although resources noted the benefits $(88 \%)$ and risks (55\%) of osteoporosis drugs, only two resources reported these with equal detail $(22 \%)$.

Discussion \& Conclusion: The majority of osteoporosis patient information resources do not meet basic readability levels, rendering them too complex for a large proportion of the UK population ( $43 \%$ of working age adults). None of the resources included in this study scored higher than $70 \%$ using the m-IPDAS quality assessment, illustrating problems relating to accuracy, comprehensive coverage, balance, and bias. Our ongoing work with clinical and patient partners will produce recommendations for improving quality.

\section{Abstract ID: 42 \\ Original Research \\ Subcategory: Patient experience}

\section{An exploratory qualitative study looking at the views and knowledge of osteoporosis sufferers on sedentary behaviour in the development and management of their disease}

\section{Grady', F Muirhead', A Mavroeidi', DA Skelton ${ }^{2}$}

1School of Psychological Sciences and Health, University of Strathclyde, Glasgow, UK, ${ }^{2}$ Department of Physiotherapy and Paramedicine, Glasgow Caledonian University, Glasgow, UK

Background: There is evidence linking higher levels of sedentary behaviour (SB) and the onset of chronic illnesses (e.g. diabetes, cardiovascular disease, and all-cause mortality). However, there is only a postulated link between SB and bone health. Co-creation (where the target population actively participate in the conception and creation of the intervention) has been suggested as a collaborative and innovative approach to create new feasible and sustainable lifestyle interventions.

Objectives: (1) To develop an understanding of the thoughts and opinions of older adults (OA) suffering from osteoporosis (OP) regarding SB and (2) investigate strategies used to reduce SB for intervention development. 
Methods: Twelve OA (of which 11 were female) with OP [mean age $=68.4 \mathrm{y} \pm 6.3(\mathrm{SD})$ ] participated in semi-structured interviews (March-May 2020) from the Scottish section of the Royal Osteoporosis Society support group networks and the Strathclyde Age-Friendly-Academy. Telephone interviews were recorded, transcribed verbatim and thematically analysed using Braun $\&$ Clarkes (2006) six steps to data analysis.

Results: Five main and seventeen sub themes emerged: 'OA Knowledge', 'Interrupting SB', 'Motivators to reduce SB', 'OP-related lifestyle changes', and 'OA and Technology'. OA had a good understanding of what $\mathrm{SB}$ is and how it affects health and OP. They felt there was not enough encouragement to break up SB in society and mainly relied on self-motivation. Participants identified facilitators of SB (e.g. Television), motivators to interrupt SB (e.g. Ad-breaks) and their awareness of prolonged periods of sitting. They reported an increase/maintenance of PA levels after OP diagnosis, but had to consider safety measures to prevent slips, trips and falls. Majority of participants appeared to use technology to track their PA and SB (e.g. Fitbit) but were also aware that access and usability are potential barriers when using technology to reduce SB among OA.

Discussion: Older OP sufferers are aware of their own SB levels, and what they can do to reduce periods of inactivity however, there is 'no blanket effect' that is, each case of OP is different and is subject to review.

Conclusion: These results could help future cocreated interventions to stimulate a campaign to reduce $\mathrm{SB}$ among in this population subgroup.

\section{Abstract ID: 43 \\ Original Research \\ Subcategory: DXA}

\section{Job stress and bone mineral density in the Hertfordshire cohort study}

MA Clynes' ${ }^{1}$ S D'Angelo ${ }^{1}$, C Cooper $^{1,2}$, KE Walker-Bone', EM Dennison ${ }^{1}$

${ }^{1}$ MRC Lifecourse Epidemiology Unit, University of Southampton, Southampton, UK, ${ }^{2}$ NIHR Oxford Biomedical Research Centre, University of Oxford, Oxford, UK

Background: Individuals with high levels of selfperceived stress have been reported to have an increased risk of any osteoporotic fracture, in particular at the hip, even after adjusting for confounders. Here we consider the relationships between job stress and bone mineral density (BMD) in participants from the Hertfordshire Cohort Study (HCS).

Objective: Here we consider the relationships between job stress and bone mineral density (BMD), in participants from the Hertfordshire Cohort Study (HCS).

Methods: Study participants in their seventh decade completed a social health questionnaire which detailed lifestyle factors and employment related DC and ERI. Femoral neck and lumbar spine BMD was measured by dual energy X-ray absorptiometry (DXA) using a Hologic QDR 4500 instrument. The characteristics of men and women reporting either or both work stresses were compared, and the association of these with femoral neck BMD and lumbar spine explored.

Results: Data were available for 390 men (mean age 64.1 years) and 357 women (mean age 65.6 years), reporting on their most recent or current job. A total of $241(61.8 \%)$ men and 211 $(59.1 \%)$ women reported neither work stress whilst $52(13.3 \%)$ men and $44(12.3 \%)$ women reported both. Mean (SD) BMD at the femoral neck was higher in men than women $(0.85(0.12)$ and $0.75(0.12)$ respectively). Women reporting both work stresses had significantly higher femoral neck BMD compared with those reporting neither [average difference in $\mathrm{g} / \mathrm{cm}^{2}(95 \% \mathrm{CI})$ $0.04(0.0002,0.09)]$. This association was attenuated after adjustment for BMI. Lumbar spine BMD was not associated with either or both work stress in either sex, before or after adjustment for confounders (age, age when ended education, marital status, smoking, alcohol and body mass index (BMI)).

Discussion: Women reporting higher levels of work stress had higher femoral neck BMD, though adjustment for BMI attenuated this association, suggesting associations were mediated through increased adiposity in this group.

Conclusion: Work stress is known to be associated with cardiovascular mortality and morbidity and it appears that metabolic changes triggered by work stress may also increase adiposity and increase femoral neck BMD. 


\section{Abstract ID: 45 \\ Original Research \\ Subcategory: FLS}

\section{Exploring experiences of fracture liaison services and the role of a consultation intervention: a focus group study}

\section{Bullock ${ }^{1}$, C Jinks ${ }^{1}$, A Hawarden 1,2, F Manning- Crawford $^{1,2}$, J Fleming ${ }^{3}$, E Clark $^{4}$, C Iglesias $^{5}$, T O'Neill6, C Mallen', Z Paskins ${ }^{1,2}$ \\ ${ }^{1}$ School for Primary, Community, and Social Care, Keele University, Newcastle-under-Lyme, UK, ${ }^{2}$ Haywood Academic Rheumatology Centre, Staffordshire and Stoke-on-Trent Partnership Trust, Stoke-on-Trent, UK, ${ }^{3}$ Cambridge Institute of Public Health, University of Cambridge, Cambridge, UK, ${ }^{4}$ Faculty of Health Sciences, University of Bristol, Bristol, UK, ${ }^{5}$ Department of Health Sciences, University of York, York, UK, ${ }^{6}$ Centre for Epidemiology Versus Arthritis, University of Manchester, Manchester, UK}

Background: National osteoporosis guidance recommends the provision of information as a core component of management. Effective use of information within a consultation increases patient satisfaction and commitment to treatment. The iFraP intervention consists of a computerised decision-support tool (DST) and clinician training package to facilitate communication and shared decision-making (SDM), with a long-term aim of improving informed medication adherence.

Objective: To inform intervention development, we explored (1) current experiences of Fracture Liaison Service (FLS) appointments and (2) perspectives towards the new iFraP intervention.

Methods: Two focus groups were conducted with FLS healthcare professionals (HCPs), one with General Practitioners (GPs), and one with patients who recently attended an FLS appointment. Supplementary patient interviews were also conducted. Theoretically-informed thematic analysis was conducted to facilitate understanding of current FLS practice, potential intervention acceptability and possible barriers to, and facilitators of, implementation.

Results: FLS HCPs and GPs suggested that current FLSs worked well to identify and recommend osteoporosis treatment. However, many patients described their unmet need for more information about drugs. FLS consultations varied in content: some FLS HCPs did not view osteoporosis medicines as their responsibility and did not discuss medicines with the patient, with others discussing medicines in face-to-face clinic(s), by letter, or in the patient's home. All FLS HCPs described a number of uncertainties (e.g. if the patient had osteopenia) when determining if a patient should be recommended osteoporosis medicine. Overall, patients and GPs were confident that the iFraP intervention could meet patients' need for information by using visual images to facilitate understanding of osteoporosis. Many FLS HCPs reflected upon the value of the intervention (e.g. the use of Cates plots to support explanations of osteoporosis medicine effectiveness) but also potential barriers to intervention implementation.

Discussion: For the first time, these findings illustrate the experience of FLS consultations from three perspectives. The findings highlight FLS HCPs' clinical decision-support needs and patients' unmet need for clear information that addresses their concerns about medication.

Conclusion: The iFraP intervention was viewed as having potential to support HCPs' decisionmaking, and to facilitate communication and SDM. Differences in FLS configuration may constitute a barrier to implementation.

\section{Abstract ID: 47 \\ Original Research Subcategory: FLS}

What is important to include in a fracture liaison service consultation?: a Delphi survey to inform the development of the iFraP intervention

\section{Bullock ${ }^{1}$, C Jinks ${ }^{1}$, F Manning-Crawford 1,2,

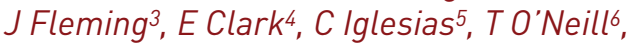 C Mallen ${ }^{1}$, Z Paskins ${ }^{1,2}$}

${ }^{1}$ School of Primary, Community and Social Care, Keele University, Newcastle-under-Lyme, UK, ${ }^{2}$ Haywood Academic Rheumatology Centre, Staffordshire and Stoke-on-Trent Partnership Trust, Stoke-on-Trent, UK, ${ }^{3}$ Cambridge Institute of Public Health, University of Cambridge, Cambridge, UK, ${ }^{4}$ Faculty of Health Sciences, University of Bristol, Bristol, UK, ${ }^{5}$ Department of Health Sciences, University of York, York, UK, ${ }^{6}$ Centre for Epidemiology Versus Arthritis, Faculty of Biology, Medicine and Health, University of Manchester, Manchester, UK

Background: Evidence-based treatments are recommended for patients with osteoporosis, however, many patients do not start or persist with treatment.

Objective: This study aimed to inform iFraP (Improving uptake of Fracture Prevention drug Treatments) intervention development, consisting 
of (1) a computerised decision-support tool, and (2) clinician training to improve informed fracture prevention treatment adherence.

Methods: Two rounds of modified Delphi survey were sent to patients with osteoporosis and/or fragility fractures, carers, and healthcare professionals (HCPs). Participants were presented with potential iFraP intervention content, derived from an evidence synthesis of current osteoporosis guidelines, theories of communication and stakeholder consultation. Participants were asked to rate their perception of the importance of each item/task and elaborate using free-text boxes. In Round 2, participants were shown mean scores of importance from Round 1. Items/tasks identified as of 'low importance' were removed before the subsequent round.

Results: In both Rounds, the majority of items were rated as important. In Round $1(n=72)$, items describing the consequences of osteoporosis (e.g. 'one in ten patients with a hip fracture will die within 12 months of fracture') were scored as low importance, with concerns that such statements may induce fear. HCP ratings indicated that examinations to identify spinal fractures were of low importance, with free-text responses suggesting that Fracture Liaison Service (FLS) HCPs may not 'be best qualified' to perform examinations. In Round $2(n=49)$ items using frequencies to explain the risk of osteoporosis, or the benefits/side effects of medicines (e.g. the risk of breaking a hip over the next 10 years) were deemed of low importance. When exploring which medications FLS HCPs should be able to discuss, Hormone Replacement Therapy, Raloxifene and Teriparatide injections were of low importance, with free-text statements suggesting that 'specialists' (e.g. Rheumatologists) should discuss these treatments rather than FLS HCPs due to their unfamiliarity.

Discussion: HCPs should provide patients with understandable information that highlights the importance of osteoporosis treatment, whilst remaining mindful of avoiding fearful messages. Patients and HCPs should be supported to discuss osteoporosis treatments, including their benefits and risks.

Conclusion: The Delphi survey has informed iFraP intervention development by highlighting important content.

\section{Abstract ID: 49 \\ Original Research \\ Subcategory: Management and treatment}

\section{${ }^{*}$ Discharge after hip fracture surgery by mobilisation timing: secondary analysis of the UK national hip fracture database}

\begin{abstract}
A Goubar ${ }^{1}, 0$ Almilaji', FC Martin 1,2, C Potter2, GD Jones², C Sackley1, S Ayis', KJ Sheehan'

'Department of Population Health Sciences, School of Population Health \& Env, King's College London, London, UK, '2Guy's and St. Thomas's National Health Service Foundation Trust, London, UK
\end{abstract}

Background: To maximise the benefits of hip fracture surgery the National Institute for Health and Care Excellence Clinical Guideline recommends mobilisation on the day after hip fracture surgery based a low to moderate quality trial with a small sample size. There is a need to generate additional evidence to support early mobilisation as a new UK Best Practice Tariff (BPT).

Objective: To determine whether mobilisation timing was associated with the cumulative incidence of hospital discharge by 30-days after hip fracture surgery, accounting for potential confounders and the competing risk of in-hospital death.

Method: We examined data for 135,105 patients 60 years or older who underwent surgery for nonpathological first hip fracture between January 2014 and December 2016 in any hospital in England or Wales. We tested whether the cumulative incidences of discharge differed between those mobilised early (within 36 hours of surgery) and those mobilised late accounting for potential confounders and the competing risk of in hospital death.

Results: 106,722 (79\%) of patients first mobilised early. The average rate of discharge was 60.1 (95\% CI 59.8-60.5) per 1000 patient days, varying from 65.2 (95\% CI 64.8-65.6) among those who mobilised early to 44.5 (95\% CI 43.9-45.1) among those who mobilised late, accounting for the competing risk of death. By 30 days postoperatively, the crude and adjusted odds ratios of discharge were 2.26 (95\% CI 2.2-2.32) and 1.93 (95\% CI 1.86-1.99) respectively among those who first mobilised early compared to those who mobilised late, accounting for the competing risk of death.

Discussion: Early mobilisation led to a near two fold increase in the adjusted odds of discharge by 30-days postoperatively. In the current 
study, one-fifth of patients experienced delayed mobilisation. Reasons for delay may relate to surgi$\mathrm{cal}$ and/or anaesthetic care process, or rehabilitation structures of care, which may be amenable to change to reduce unwarranted variation in mobilisation timing.

Conclusion: We recommend inclusion of mobilisation within 36 hours of surgery as a new UK $\mathrm{BPT}$ to encourage sites to target these care processes and help reduce delays to mobilization.

\section{Abstract ID: 54 \\ Original Research \\ Subcategory: Fracture risk \\ Low trauma fragility fractures presenting to a regional spinal unit}

\section{A Tarawneh, N Sadanandan, K Salem, O Sahota Queen \& Medical Centre, Nottingham University Hospitals NHS Trust, Nottingham, UK}

Background: With ageing demographics, the number of older people is expected to rise exponentially over the next decade. Low trauma fragility fractures are particularly prevalent in older people and commonly occur following a fall from standing height or less. Many of these sustain with vertebral and sacral fractures and present to regional spinal services.

Objective: Identify the incidence of low trauma fragility fractures presenting to the Nottingham Regional Spinal unit for patients aged 70 years and above over a 30-month period.

Methods: A retrospective 30-month review (January 2017-August 2019) of the clinical records of all in-patient referrals to the regional spinal unit (catchment population 4.5 million) for patients aged 70 years and above was undertaken. Patient demographics, co-morbidities, cause of referral, treatment modality (conservative versus surgical), length of hospital stay, discharge destination (home versus rehab) and mortality rates were collected and analysed.

Results: A total of 677 (Male: 335, Female: 342) in-patient referrals for patients aged 70 years and above were received. The mean age at presentation was $82.3( \pm 7.48)$ years.

Trauma [low trauma (LT) \& high trauma (HT)] was the most common cause of inpatient referrals $(n=448 ; 66.2 \%)$. Low trauma fragility fractures (LT) was the leading cause for the referrals and contributed to $37.8 \%(n=256)$ of the total inpatient referrals. HT contributed to $28.3 \%$ of the referrals $(n=192)$. The mean age of presentation of the LT group was $(83.9 \pm 7.1)$ years similar to (83.3 \pm 7.3$)$ years in the HT group. Two-hundred and six LT fractures were treated conservatively (80.4\%) compared to $89.1 \%(n=171)$ in HT group. Average length of hospital stay was 16.2, 12.8 days for LT and HT, respectively. Thirtyseven of the discharged patients (15.5\%) in LT were transferred to rehabilitation after discharge in comparison to $14.4 \%(n=22)$ in HT. Over-all 30 -day mortality rate was $6.6 \%$ for LT and $20.3 \%$ for HT.

Conclusion: A significant proportion of patients present to a regional spine unit with low trauma fragility fractures and are at high risk of future fragility fractures. These units require a dedicated fracture liaison service.

\section{Abstract ID: 58 \\ Original Research \\ Subcategory: Hip fracture}

\section{Causes of death after hip fracture in Norway}

K Holvik', CL Ellingsen², SM Solbakken², TE Finnes ${ }^{4,5}, 0$ Talsnes ${ }^{6}$, G Grimnes ${ }^{7,8}$, GS Tell?, AJ Søgaard ${ }^{1}$, HE Meyer ${ }^{1,3}$

1Department of Chronic Diseases and Ageing, Norwegian Institute of Public Health, Oslo, Norway, ${ }^{2}$ Department of Pathology, Stavanger University Hospital, Stavanger, Norway, ${ }^{3}$ Department of Community Medicine and Global Health, University of Oslo, Oslo, Norway, 4Department of Endocrinology, Oslo University Hospital, Oslo, Norway, ${ }^{5}$ Department of Internal Medicine, Innlandet Hospital Trust, Hamar, Norway, ${ }^{6}$ Department of Orthopaedic Surgery, Innlandet Hospital Trust, Elverum, Norway, ${ }^{7}$ Department of Clinical Medicine, UiT The Arctic University of Norway, Troms $\varnothing$, Norway, ${ }^{8}$ Division of Internal Medicine, University Hospital of Northern Norway, Troms $\varnothing$, Norway, ${ }^{9}$ Department of Global Public Health and Primary Care, University of Bergen, Bergen, Norway

Background: Hip fracture is a serious injury particularly affecting older patients with multimorbidity. Information on causes of death helps appraise the degree to which the high excess mortality in these patients reflects pre-existing comorbidities versus the hip fracture trauma itself.

Objective: To describe the distribution of causes of death after hip fracture in Norway.

Methods: Hospital information on all hip fractures 1999-2013 was available. Diagnoses for underlying causes of death through 2017 , coded 
according to ICD-10, were obtained by linkage to the Norwegian Cause of Death Registry, and grouped in categories defined by the EU shortlist. We calculated incidence proportions of mortality by cause of death categories according to time since the hip fracture. Additional analyses were performed in strata of sex, age and time period.

Results: Among 122,199 patients aged 50 years and older who suffered an incident hip fracture, $24.3 \%$ died within 1 year. External causes of injury, dominated by a diagnosis of unspecified fall or exposure to unspecified factor, comprised the leading underlying cause of death up to 6 months, overtaken by circulatory diseases for longer-term deaths ( $<1$ year and $<4$ years). Within 1 year, $6.3 \%$ had died from external causes and $6.8 \%$ from circulatory diseases. The corresponding incidence proportions in hip fracture patients 85 years and older were $10.7 \%$ and $10.3 \%$, respectively, out of $34.4 \%$ one-year deaths. Other common cause of death categories were, in descending order; cancer, respiratory diseases, and mental disorders (mainly dementia).

Discussion: External causes of injury and circulatory diseases were the most common underlying causes of death, each causing one-fourth of 1 -year deaths in the hip fracture patient population, and almost one-third in the oldest patients. With increasing time after the fracture, the distribution of causes of death in hip fracture survivors converged towards the pattern in the general population.

Conclusion: The traumatic injury of a hip fracture is a leading underlying cause of death among older patients who survive less than 1 year after their fracture. This finding underlines the importance of preventing falls and fractures in the older population.

\section{Abstract ID: 59 \\ Original Research \\ Subcategory: Hip fracture}

To what extent does achievement of the best practice tariff explain between-hospital variation in hip fracture outcomes?

\footnotetext{
R Patel', A Judge1, Y Ben-Shlomo², CL Gregson'

${ }^{1}$ Musculoskeletal Research Unit, University of Bristol, Bristol, UK,

2Population Health Sciences, University of Bristol, Bristol, UK
}

Background: Each year in England over 60,000 older adults fracture a hip, impacting quality-oflife, survival and health costs. Fracture services are provided through complex multidisciplinary organisational structures. Despite UK standards and guidelines, hospital delivery of hip fracture care is highly variable, as are patient outcomes across the country.

Objective: To determine the impact of organisational factors on the variation in hip fracture outcomes between English hospitals.

Methods: Analysis of National Hip Fracture Database (NHFD), Hospital Episodes Statistics (HES) and Office for National Statistics (ONS) mortality data for $2014 / 15$, linked at the patientlevel, and combined with Best Practice Tariff (BPT) organisational-level audit data. Statistical analyses used multi-level models, to identify which BPT organisational factors are responsible for the greatest variation in two patient outcomes: 30-day mortality and 'superspell' length of hospital stay (SLOS).

Results: We analysed 51,714 patient records from 162 English hospitals. Documentation of pre-operative Abbreviated Mental Test Score (AMTS) associated with lower mortality risk [OR 0.74 (95\% CI 0.58, 0.94); $p=0.02$ ], other components of BPT did not substantially change mortality risk. Substantial between-hospital variation in 30-day mortality persisted from $2.7 \%$ to $7.5 \%$ (Figure 1 ) after adjusting for within-hospital clustering, patient case-mix and BPT factors. Factors predicting reduced SLOS were documented pre-operative AMTS $(p=0.08)$ and physiotherapy assessment $(p=0.08)$. After full adjustment, substantial between-hospital

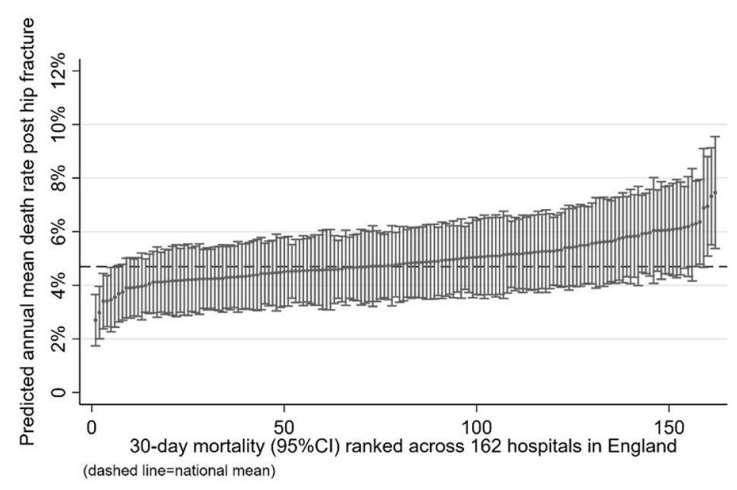

Figure 1. Variability in 30-day mortality, adjusted for patient case mix and hospital BPT parameters across English hospitals 
variation in SLOS remained varying from 13.1 to 39.8 days.

Discussion: We identified important BPT parameters which predict reduced mortality and length-of-stay, independent of patient characteristics. Although these parameters could explain variation in outcomes between patients, they explained little of the variation in outcomes between hospitals.

Conclusion: Understanding sources of variation in care delivery and its effects on patient outcomes, may inform service-level interventions to reduce unwarranted variation and maximise health equity.

\section{Abstract ID: 61 \\ Original Research \\ Subcategory: Hip fracture}

The contribution of an extensive medicationbased comorbidity index to explain excess mortality after hip fracture in Norway

\footnotetext{
K Holvik', V Hjellvik', Ø Karlstad' ${ }^{1}, N$ Gunnes$^{2}, M$ Hoff $3,4,5$, GS Tell 6 , HE Meyer ${ }^{1,7}$

${ }^{1}$ Department of Chronic Diseases and Ageing, Norwegian Institute of Public Health, Oslo, Norway, ${ }^{2}$ Norwegian National Advisory Unit on Women's Health, Oslo University Hospital, Oslo, Norway, ${ }^{3}$ Department of Neuromedicine and Movement Science, Norwegian University of Science and Technology, Trondheim, Norway, ${ }^{4}$ Department of Public Health and Nursing, Norwegian University of Science and Technology, Trondheim, Norway, ${ }^{5}$ Department of Rheumatology, St Olavs Hospital, Trondheim, Norway, ${ }^{6}$ Department of Global Public Health and Primary Care, University of Bergen, Bergen, Norway, ${ }^{7}$ Department of Community Medicine and Global Health, University of Oslo, Oslo, Norway
}

Background: Methods to account for a wide range of comorbidities in population-wide register-based studies are needed. In national prescription databases, information on drug use is available for the general population.

Objective: To apply the existing Rx-Risk Comorbidity Index based on dispensed drugs and adapt it to treatment practices in Norway, investigate its association with mortality according to history of hip fracture in the older population, and quantify its contribution in explaining the excess mortality after hip fracture.

Methods: The Rx-Risk Comorbidity Index was based on dispensed drugs recorded in the Norwegian Prescription Database (NorPD). Hospital data provided information on all hip fractures in Norway 1994-2013, and Statistics Norway provided dates of death. Using 2013 as the index year for calculating individual Rx-Risk scores in the ambulatory older population, we estimated one-year risk of death by Rx-Risk score, history of hip fracture, age and sex in linear regression.

Results: The NorPD population aged 65 years and older who were alive by the end of 2013 comprised 735,968 individuals, of whom 698,076 (94.9\%) filled at least one prescription within one or more of the $46 \mathrm{Rx}$-Risk comorbidity categories in 2013. $\mathrm{Rx}$-Risk scores ranged from -5 to 44 and were higher in those with a history of hip fracture: mean score 6.9 , versus 4.4 in those who had not experienced a hip fracture. One-year deaths increased with increasing $\mathrm{Rx}$-Risk scores and were higher in those with a history of hip fracture. When not considering Rx-Risk scores, age- and sex-adjusted oneyear mortality was $7.8 \%$ in those with a history of hip fracture and $3.4 \%$ in those with no hip fracture (risk difference 4.4 percentage points). When including adjustment for the Rx-Risk scores, the estimated risk difference was attenuated from 4.4 [95\% confidence interval (CI): 4.2, 4.6] to 3.3 (95\% CI: 3.1, 3.5) percentage points.

Conclusion: Individual comorbidity scores calculated by the Rx-Risk Comorbidity Index based on dispensed drugs explained a quarter of the excess mortality in those with a history of hip fracture, when also accounting for sex and age distribution.

\section{Abstract ID: 62 \\ Original Research Subcategory: DXA}

*Differential relationships between parentchild DXA and pQCT bone measures: results from the Southampton women's survey

\section{SA Carter ${ }^{1}$, CR Holroyd ${ }^{1,2}$, SR Crozier ${ }^{1}$, K Ward $^{1}$, EM Dennison', HM Inskip 1,3, KM Godfrey1,3, C Cooper ${ }^{1,3,4}$, NC Harvey ${ }^{1,3}$ \\ 1MRC Lifecourse Epidemiology Unit, University of Southampton, Southampton, UK, ${ }^{2}$ Rheumatology Department, University Hospital Southampton NHS Foundation Trust, Southampton, UK, ${ }^{3} \mathrm{NIHR}$ Southampton Biomedical Research Centre, University of Southampton and University Hospital Southampton NHS Foundation, Southampton, UK 4NIHR Biomedical Research Centre, University of Oxford, Oxford, UK}

Background and Aim: To investigate the associations between indices of bone mass in childhood and corresponding parental measures.

Methods: The Southampton Women's Survey cohort initially comprised 12,583 non-pregnant 
women aged 20-34 years, of whom 3158 had subsequent singleton live births, who have been followed-up. In a subset of participants, dual-energy $\mathrm{x}$-ray absorptiometry (DXA) assessment of bone mass was obtained in the child (at 8-9years) and in both parents, yielding bone area (BA), bone mineral content (BMC) and areal bone mineral density (aBMD) at lumbar spine and total hip sites. Another subset of children, at 6-7 years, and their parents, had peripheral quantitative computed tomography (pQCT; $4 \%$ and 38\% tibia) measures. Multivariable linear regression was used to assess relationships between mother/ father and offspring, adjusting for that parent's age, height, habitual walking speed and social class; offspring age and height, and sex; and the corresponding bone measure in the other parent ( $\beta$ coefficients as SD per SD).

Results: Data were available for 260 trios with DXA and 99 with pQCT. There were positive associations for BA, BMC and aBMD between either parent and offspring (shown here for lumbar spine), $\beta$ ranging from 0.06 to $0.25 \mathrm{SD} / \mathrm{SD}$. Mother-child associations were of greater magnitude than father-child: For example, mother-child aBMD $(\beta=0.25,95 \%$ CI $0.19,0.30)$ and fatherchild aBMD $(\beta=0.13,95 \%$ CI: $0.07,0.20)$, P-difference in $\beta=0.001$. The pattern of associations was less clear for pQCT in this small subset. There was a positive association for mother-offspring $4 \%$ tibial total area $(\beta=0.293,95 \%$ CI $0.145,0.440)$, whereas there was little evidence of a father-offspring association $(\beta=-0.080,95 \%$ CI: $-0.220,0.065)$. Parent-offspring associations were of similar magnitude for $38 \%$ cortical density (mother-offspring: $\beta=0.325,95 \%$ CI 0.010 , 0.640 ; father-offspring: $\beta=0.472,95 \%$ CI 0.103 , $0.841)$.

Conclusions: Whilst offspring bone measures are independently associated with those of either parent, the magnitude of the association is greater for maternal than paternal relationships. These findings are consistent with an in utero influence on offspring growth but might also reflect genetic and epigenetic parent of origin effects.

\section{Abstract ID: 63 \\ Original Research \\ Subcategory: Fracture risk
Body height and the risk of hip fracture in Women

HE Meyer ${ }^{1,2}, C$ Dahl2 $^{2}$, HK Kjeldgaard 1 , B Schei3, AJ Søgaard ${ }^{1}$, GS Tell', K Holvik'

'Department of Chronic Diseases and Ageing, Norwegian Institute of Public Health, Oslo, Norway, ${ }^{2}$ Department of Community Medicine and Global Health, University of Oslo, Oslo, Norway, ${ }^{3}$ Department of Public Health and Nursing, Norwegian University of Science and Technology, Trondheim, Norway, ${ }^{4}$ Department of Global Public Health and Primary Care, University of Bergen, Bergen, Norway

Background: Several studies have reported a positive association between body height and increased risk of hip fracture, whereas others have not. The lack of association in some studies could be due to age-related height loss, which may be more prominent in those with osteoporosis. This may be investigated by applying information about the individuals' highest attained body height in young adult age.

Objective: To assess if body height measured three decades earlier is more strongly related to the risk of hip fracture compared to current height in elderly women.

Methods: Seven Norwegian regional community based health studies were carried out in the period 1995 to 2002 with similar data collection, including standardized height and weight measurements (hereafter called examination 2). The analysis was restricted to 6516 women aged $65-79$ years at examination 2 (mean age $70.3 \pm 3.1$ years) who also had their height and weight measured in a nationwide tuberculosis screening program during 1963-75 when mean age was $39.8 \pm 3.3$ years (examination 1). We excluded women aged $<20$ years or $\geqslant 45$ years at examination 1 . The participants were followed with respect to incident hip fracture from examination 2 throughout 2013 using Cox regression.

Results: 912 women sustained a hip fractures during follow-up. A $10 \mathrm{~cm}$ higher body height was associated with a $26 \%$ increased risk of hip fracture $(\mathrm{HR}=1.26(95 \%$ CI $1.12-1.41)$ based on body height measured at examination 2. However, when height measured at examination 1 was used, $10 \mathrm{~cm}$ higher height was associated with a $44 \%$ higher risk $[\mathrm{HR}=1.44(95 \%$ CI 1.28-1.62)].Compared to women in the lowest quintile of body height at examination $1(<158 \mathrm{~cm})$, those in the highest quintile $(\geqslant 168 \mathrm{~cm})$ had an $87 \%$ increased risk of hip fracture $[\mathrm{HR}=1.87$ (95\% CI 1.50-2.34)].

Conclusion: Body height is a risk factor for hip fracture, which may easily be overlooked if current height in elderly people are used instead of their young adult height. 


\section{Abstract ID: 65 \\ Original Research \\ Subcategory: Fracture risk}

Previous falls indicate risk of future hip fracture among those with poor general health

\author{
R Aga' , AJ Søgaard', K Holvik', HE Meyer ${ }^{1,2}$ \\ 'Department of Chronic Diseases and Ageing, The Norwegian Institute of \\ Public Health, Oslo, Norway, ${ }^{2}$ Faculty of Medicine, University of Oslo, Oslo,
} Norway

Background: Elderly with previous falls have higher risk of recurrent falls, but the group is heterogeneous. What factors should health care professionals who meet elderly patients with fall injuries look for when assessing their future fracture risk?

Objective: Does general health among elderly who have experienced falling, influence their risk of a future hip fracture?

Methods: A population-based prospective cohort study was used. Information on self-perceived general health and falls in the year preceding the survey was obtained from questionnaires in a collection of regional health studies with harmonized data performed in Norway during the years 2000-2002. A total of 7751 men and women aged 70-79years old were followed throughout 2013 with respect to hip fracture by linkage to a database including all hip fractures treated in Norway. Cox survival analysis was performed to estimate hazard ratios (HR) for hip fracture according to self-reported previous falls and self-perceived general health.

Results: At baseline, $28.8 \%$ of the whole group reported that they had fallen during the last year. During follow-up, 985 individuals, comprising of $303(8.7 \%)$ of the men and $682(16.0 \%)$ of the women, sustained a hip fracture. In men, those who reported poor general health and who also reported one or more falls during the previous year, had a HR of 2.29 (95\% CI 1.66-3.15) of hip fracture compared to healthy non-fallers. The corresponding figure in women was $\mathrm{HR}$ of 1.62 (CI 95\% 1.33-1.99). In contrast, women and men with self-perceived good health who reported falls in the preceding year did not have an increased risk of future hip fracture compared to healthy non-fallers.

Discussion: The information given in the study may have changed at the time of the fracture.
Furthermore, there is no information on the circumstances around the fall incidents. Conclusion: Elderly in poor health, and men in particular, who report previous falls are at high-risk of a future hip fracture. An increased fracture risk was not observed in healthy fallers.

\section{Abstract ID: 66 \\ Original Research \\ Subcategory: Vertebral fracture}

\section{Prevalence of osteoporotic vertebral fractures in older women with back pain and impact on health related quality of life}

T Khera', Z Paskins'2,3, JH Tobias', $R$ Gooberman-Hill', TJ Peters', S Davis4, A Burston', EM Clark ${ }^{1}$

${ }^{1}$ Bristol Medical School, University of Bristol, Bristol, UK, ${ }^{2}$ Arthritis Research UK Primary Care Centre, Keele University, Keele, UK, ${ }^{3}$ Haywood Academic Rheumatology Centre, Midlands Partnership NHS Foundation Trust, Stoke-on-Trent, UK, 4Health Economics and Decision Science, University of Sheffield, Sheffield, UK

Background: Despite good population-based data on the prevalence of osteoporotic vertebral fractures (OVFs) in the general population, little is known about the prevalence and impact of OVFs in those with back pain.

Objective: To identify the prevalence of OVF in a cohort of older women with back pain, and to quantify impacts on health-related quality of life (HRQoL), including pain and function.

Methods: This observational study is a cohort of women aged $>65$ years with self-reported back pain in the preceding 4 months, originally recruited to develop a tool to screen for OVFs. This analysis uses baseline data. All participants had spinal radiographs, assessed for the presence or absence of OVF using the algorithm-based qualitative approach. HRQoL was assessed using the EQ-5D (EQ-VAS, pain item, and mobility item), falls and use of walking aids (including wheelchairs). Socio-economic status (SES) was assessed using highest achieved education. Analyses were based on a multivariable logistic regression approach. Ethics approval was obtained from the West of Scotland REC 3 18/ WS/0061.

Results: Out of 1635 participants, 134 (8.2\%) had one OVF and 75 (4.6\%) had $>1$ OVF. There was no difference in EQ-VAS nor EQ5D pain sub-score in those with or without OVFs. $40.0 \%$ 
of those with $>1$ OVF used a walking aid compared to $28.5 \%$ without OVFs $(p<0.001)$, robust to adjustment for area, age and SES. Those with $>1$ OVF had a worse EQ-5D mobility sub-score than those with no OVF (mean \pm SD $2.47 \pm 1.00$ versus $2.01 \pm 1.01, p=0.002)$. Those with $>1$ OVF had more falls than those with only one $\mathrm{OVF}$, but this association was no longer present after adjusting for age and SES.

Discussion: $12.8 \%$ of older women with back pain had at $\geqslant 1 \mathrm{OVF}$ and should be targeted for screening. The burden of disease was greater for those with more than one OVF, particularly related to mobility and need for walking aids. Interventions to improve HRQoL in those with back pain should be prioritised in those with OVFs.

\section{Abstract ID: 67 \\ Original Research \\ Subcategory: Vertebral fracture}

\section{Knowledge of presence of osteoporosis and/ or vertebral fracture and healthcare usage: results from a cohorts of older women with back pain}

T Khera' , Z Paskins 2,3, JH Tobias', R Gooberman-Hill', TJ Peters', S Davis', A Burston', EM Clark'

${ }^{1}$ Bristol Medical School, University of Bristol, Bristol, UK, ${ }^{2}$ Arthritis Research UK Primary Care Centre, Keele University, Keele, UK, ${ }^{3}$ Haywood Academic Rheumatology Centre, Midlands Partnership NHS Foundation Trust, Stoke-on-Trent, UK, 4Health Economics and Decision Science, University of Sheffield, Sheffield, UK

Background: Most osteoporotic vertebral fractures (OVFs) do not present to healthcare, but the actual proportion of older women with undiagnosed OVFs is unknown. Also, healthcare costs associated with OVFs are increasing as our population ages, but it is unknown if this is influenced by knowledge of a formal diagnosis.

Objective: To identify the proportion of older women with OVF who remain undiagnosed, and to quantify healthcare usage (use of pain relief medication and visits to healthcare professionals) in those who are, and are not, aware of their diagnosis.

Methods: This observational study is a cohort of 1635 older women with back pain and spinal radiographs. All images were categorised as having or not having an OVF using the algorithmbased qualitative method. Self-completion questionnaires captured data on self-reported diagnosis of osteoporosis/osteopaenia and/or vertebral fractures, plus visits to healthcare professionals over the past 3 months and use of pain relief medication over the past week. Use of pain relief was scored from 0 (not taken) to 6 (taken 4-6 times a day). Additional data included age, education and concomitant illnesses. Analyses were based on multivariable logistic regression. Ethics approval was obtained (West of Scotland REC 3 18/WS/0061).

Results: $48.3 \%$ of women with $\geqslant 1$ OVF selfreported a diagnosis of osteoporosis/osteopenia. $71.8 \%$ of those with $\geqslant 1$ OVF did not know they had a vertebral fracture. Presence of an OVF (whether known or unknown) was not associated with increased use of pain relief medication, but self-reporting of osteoporosis/osteopenia was (mean \pm SD $2.1 \pm 1.4$ versus $2.4 \pm 1.5$ in those without osteoporosis/osteopenia versus those with osteoporosis/osteopenia, respectively, $p=0.003$ ). Self-reported diagnosis of osteoporosis/osteopenia was also associated with more visits to healthcare professionals (mean \pm SD $4.5 \pm 5.8$ versus $3.4 \pm 3.8$ visits respectively, $p=0.002$ ) robust to adjustment for age, education and concomitant illnesses.

Discussion: Self-reported diagnosis of osteoporosis/osteopenia, but not presence of OVF (whether known or unknown), was associated with higher use of pain relief medication and contact with healthcare professionals. Understanding the reasons for this is necessary.

\section{Abstract ID: 71 \\ Original Research \\ Subcategory: Vit D}

\section{*Vitamin D supplementation in pregnancy is associated with greater offspring bone and lean mass at 4 years: findings from the mavidos randomised controlled trial}

EM Curtis', RJ Moon 1,2, S D'Angelo', SR Crozier', NJ Bishop ${ }^{3}$, JS Gopal-Kothandapani3 , S Kennedy4, A Papageorghiou 4 , R Fraser ${ }^{5}$, S Gandhi ${ }^{5}$, I Schoenmakers ${ }^{6}$, A Prentice $^{7}$, HM Inskip ${ }^{1,8}$, KM Godfrey ${ }^{1,8}$, MK Javaid ${ }^{9}$, R Eastell ${ }^{10}$, C Cooper ${ }^{1,8,9}$, NC Harvey ${ }^{1,8}$

${ }^{1}$ MRC Lifecourse Epidemiology Unit, University of Southampton, Southampton, UK, ${ }^{2}$ Paediatric Endocrinology, University of Southampton, Southampton, UK, ${ }^{3}$ Academic Unit of Child Health, University of Sheffield, Sheffield, UK, 4Nuffield Department of Women and Reproductive 
Health, University of Oxford, Oxford, UK, ${ }^{5}$ Department of Obstetrics and Gynaecology, University of Sheffield, Sheffield, UK, ${ }^{6}$ Department of Medicine, Faculty of Medicine and Health Sciences, University of East Anglia, Norwich, UK, ${ }^{7}$ MRC Nutrition and Bone Health, University of Cambridge, Cambridge, UK, ${ }^{8}$ NIHR Southampton Biomedical Research Centre, University of Southampton, Southampton, UK, ${ }^{9}$ NIHR Oxford Biomedical Research Centre, University of Oxford, Oxford, UK ${ }^{10}$ Department of Oncology and Metabolism, University of Sheffield, Sheffield, UK

Objectives: MAVIDOS is a multi-centre randomised trial of vitamin $\mathrm{D}$ supplementation in pregnancy, in which we demonstrated that the intervention (vs placebo) led to greater neonatal bone mass amongst winter-born babies. We investigated whether this beneficial effect is sustained into childhood at 4 years old.

Materials and Methods: 1134 pregnant women were randomised in three UK cities, in a doubleblind design, to $1000 \mathrm{IU} /$ day cholecalciferol or matched placebo from 14 weeks gestation to birth. At age 4 years (Participants from one city only, $n=723$ births), offspring assessments included anthropometry, whole-body dual-energy x-ray absorptiometry (DXA) [yielding whole body less head (WBLH) bone mineral content (BMC), bone mineral density (BMD), bone area (BA), lean mass and fat mass], and a maternal questionnaire was completed. T-tests or MannWhitney U tests were used to compare bone DXA outcomes by maternal randomisation group. Full ethics and MHRA approval was granted. Trial registration: ISRCTN82927713, registered $11 / 04 / 2008$.

Results: 564 children attended the 4-year visit and 452 had a useable DXA with little movement artefact. There was no difference in child weight, height or BMI between maternal randomisation groups. Pregnancy vitamin D supplementation led to greater offspring indices of bone mass compared with placebo, irrespective of season of birth, for example WBLH aBMD [mean (95\% CI): supplemented group: $0.477(0.472,0.481) \mathrm{g} / \mathrm{cm}^{2}$; placebo group: $0.470 \quad(0.466,0.475) \mathrm{g} / \mathrm{cm}^{2}$, $p=0.048]$. Associations were consistent for BMC and lean mass, but there was no effect on fat mass. There was evidence of a larger effect of the intervention in the context of lower childhood calcium intake and lower physical activity.

Conclusions: This new evidence from a large placebo-controlled, double-blind randomised trial suggests that maternal pregnancy vitamin D supplementation leads to a sustained improvement in offspring bone and muscle mass.

\section{Abstract ID: 79 \\ Original Research \\ Subcategory: Management and treatment}

\section{*Sedentary behaviour and bone turnover markers in older adults}

\begin{abstract}
A Mavroeidi', L McMichan', DA Skelton², N Owen ${ }^{3,4}$, DW Dunstan 3,5, WD Fraser ${ }^{6}$, JCY Tang 6 , S Agyapong-Badu7 , CA Greig7,8, SFM Chastin'2,9

${ }^{1}$ Physical Activity for Health, University of Strathclyde, Glasgow, UK, 2Department of Physiotherapy and Paramedicine, Glasgow Caledonian University, Glasgow, UK, ${ }^{3}$ Baker, Heart and Diabetes Institute, Melbourne, Australia, ${ }^{4}$ Centre for Urban Transitions, Swinburne University, Melbourne, Australia, ${ }^{5}$ Mary MacKillop Institute for Health Research, Australian Catholic University, Melbourne, Australia, ${ }^{6}$ Norwich Medical School, University of East Anglia, Norwich, UK, ${ }^{7}$ School of Sport, Exercise and Rehabilitation Sciences, University of Birmingham, Birmingham, UK, ${ }^{8}$ MRC-Versus Arthritis Centre for Musculoskeletal Ageing Research, University of Birmingham, Birmingham, UK, ${ }^{9}$ Department of Movement and Sports Science, Ghent University, Ghent, Belgium
\end{abstract}

Background: Physical immobility is a known risk factor for osteoporosis; deleterious effects on bone metabolism have been demonstrated in complete-bed-rest studies. Modern lifestyles promote a ubiquitous but less-severe form of immobility - sedentary behaviour (SB; time spent sitting). Population studies show that older adults self-report $8-10 \mathrm{~h} / \mathrm{d}$ of $\mathrm{SB}$, but the effects of SB on bone turnover markers have not been examined experimentally.

Objective: To examine whether extended periods of sitting can influence markers of bone turnover.

Methods: 33 participants [mean age 78.2 years (SD 7.4)] from the ACUte effects of SITting time on physiological and psychological function in older adults (ACUSIT) study completed three controlled uninterrupted sitting conditions $(1 \mathrm{~h}$, $2 \mathrm{~h}$ and $4 \mathrm{~h}$; randomised cross-over design). Venous blood samples were collected before and after each experimental condition. Markers of bone turnover - type I collagen C-telopeptide ( $\beta$ CTX; bone resorption) and procollagen type I amino-terminal propeptide (PINP; bone formation) - were measured using electrochemiluminesence immunoassays. Mixed factorial ANOVAs with repeated measures were performed, adjusted for age, gender, BMI, PTH and 25-hydroxyvitamin D.

Results: The bone-resorption marker ( $\beta$-CTX) increased significantly after sitting for $4 \mathrm{~h}$ compared to sitting for 1 or $2 \mathrm{~h}$, which remained significant after adjustment for confounders. There 


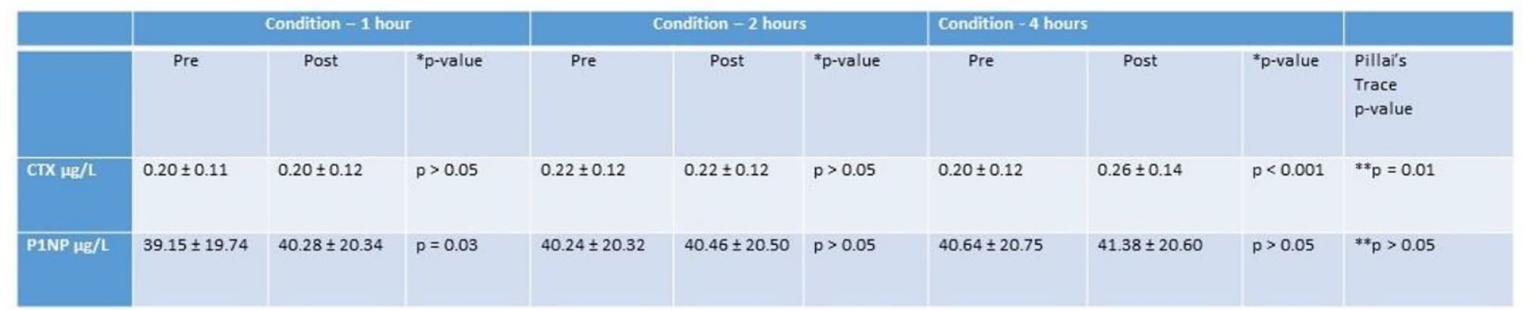

Mean $\pm \mathrm{SD} .{ }^{*}$-values were calculated using paired $\mathrm{t}$-test for each condition. ${ }^{* *}$ Multivariate Pillai's Trace for adjusted analysis (Gender, Age, BMI, 25(OH)D, PTH).

were no significant main effects for time nor condition on P1NP, apart from a small initial increase after $1 \mathrm{~h}$ of sitting.

Discussion: Longer sitting bouts led to an increase in bone resorption, while formation activity appeared to be largely unaffected. While the acute effect is of a lesser magnitude than bed rest, the accumulated chronic effect could be much higher due to the sustained continuous exposure to sitting of everyday life.

Conclusion: This is the first study to identify a dose-response acute effect of prolonged uninterrupted SB $(4 \mathrm{~h})$ on bone metabolism. Further studies, particularly identifying effects of counter-measures (frequent breaks from sitting), potentially may inform simple, evidence-based, preventive interventions for bone health.

\section{Abstract ID: 80 \\ Original Research \\ Subcategory: Vit D}

The relationship between 25-hydroxyvitamin $D$ concentration and disability trajectories in very-old adults: The Newcastle 85+ Study

\footnotetext{
SH Hakeem 1,2,3, NM Mendonca ${ }^{1,2}$, TA Aspray',5, AK Kingston 1,2, CR Ruiz-Martin'4, CJ Jagger1,2, JM Mathers ${ }^{1,2}$, RD Duncan 5 , TH Hill1,2

${ }^{1}$ Population Health Sciences Institute, Newcastle University, Newcastle, UK, ${ }^{2}$ Human Nutrition Research Centre, Newcastle University, Newcastle, UK, ${ }^{3}$ College of Nursing, Umm Al-Quraa University, Makkah, Saudi Arabia, ${ }^{4}$ BioScreening Core Facility, Faculty of Medical Sciences, Newcastle, UK, ${ }^{5}$ Feeman Hospital, NHS, Newcastle, UK
}

Background: Poor vitamin D status is common in very-old adults ( $>80$ years) and may have adverse consequences for muscle function, a major predictor of disability.

Objective: To explore the association between $25(\mathrm{OH}) \mathrm{D}$ concentrations and disability trajectories in very-old adults.
Methodology: A total of 775 participants from the Newcastle $85+$ Study for who serum $25(\mathrm{OH})$ $\mathrm{D}$ concentration at baseline was available were included. Serum 25(OH)D concentrations of $<25 \mathrm{nmol} / 1,25-50 \mathrm{nmol} / 1$ and $\geqslant 50 \mathrm{nmol} / 1$ were used as cut-offs to define poor, adequate and high vitamin $\mathrm{D}$ status, respectively. Disability was defined as difficulty in performing 17 activities of daily living, at baseline and after 18, 36 and 60 months. The relationship between the $25(\mathrm{OH})$ $\mathrm{D}$ cut-offs and disability trajectories was examined by multinomial logistic regression.

Results: A three-trajectory model was derived (low, moderate and severe). Participants with $25(\mathrm{OH}) \mathrm{D}$ concentrations $<25 \mathrm{nmol} / 1$ were more likely to have moderate $(\mathrm{OR}=2.01,95 \%$ $\mathrm{CI}=1.29-3.14, p=0.002)$ and severe $(\mathrm{OR}=3.39$, $95 \% \mathrm{CI}=1.99-5.76, p=0.001)$ disability trajectories compared with those with concentrations of $25-50 \mathrm{nmol} / 1$, after adjusting for sex, living in an institution, season, cognitive status, BMI, vitamin $\mathrm{D}$ containing medication $(\mathrm{OR}=1.97,95 \%$ $\mathrm{CI}=1.22-3.17, p=0.005)$ and $(\mathrm{OR}=3.12,95 \%$ $\mathrm{CI}=1.67-5.85, p=0.001)$, respectively. However,

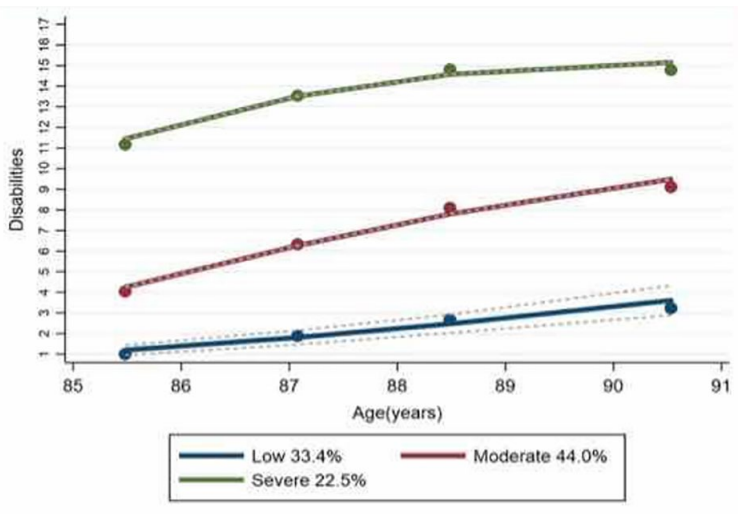

Figure 1. Disability trajectories with $95 \%$ confidence intervals in the participants

Percentages represent group size. Disabilities resulted from calculating ADLs, IADLs and mobility. 
this relationship disappeared after further adjustment for physical activity.

Discussion: In fully adjusted models trajectories of disability was not related to vitamin D status but appeared to be related to physical activity in very-old adults.

Conclusion: Physical activity rather than vitamin D status predicted disability trajectories in very-old adults.

\section{Abstract ID: 81 \\ Original Research \\ Subcategory: Fracture risk}

\section{A systematic review of observational studies reporting vitamin $D$ status in very-old adults across the world}

\section{SH Hakeem 1,2,3, WI lqbal', NM Mendonça ${ }^{1,2}$, TA Aspray ${ }^{1,4}$, TH Hill1,2 \\ 1Population Health Sciences Institute, Newcastle University, William Leech Building, Newcastle upon Tyne, UK, ${ }^{2}$ Human Nutrition Research Centre, Newcastle University, William Leech Building, Newcastle upon Tyne, UK, ${ }^{3}$ College of Nursing, Umm Al-Quraa University, Makkah, Saudi Arabia, 4 NIHR Newcastle Biomedical Research Centre, Newcastle upon Tyne Hospitals NHS Foundation Trust and Newcastle University, Newcastle upon Tyne, UK}

Background: To date, the majority of studies assessing vitamin $\mathrm{D}$ status in older adults have focused on the 'young old' or those aged less than 80 years of age. As more people reach very old age it is imperative that vitamin $\mathrm{D}$ data and its determinants become available so that public health nutrition policy can devise age-appropriate guidance for this age group.

Objective: The study aimed to assess the $25(\mathrm{OH})$ $\mathrm{D}$ concentrations in very-old adults (i.e. those $>80$ years of age) across the globe, and to explore any associations with living status and geographical locations.

Method: Four databases (Medline, ProQuest, PubMed and Web of Science) were searched for community-dwelling and institutionalised participants aged 80 years-old and older with reported serum $25(\mathrm{OH}) \mathrm{D}$ from cross-sectional studies were included. The methodology of the systematic review is based on the published protocol on PROSPERO (ID: CRD42018117158).

Results: A total of 18 studies were included in this systematic review. Four studies were from the USA, nine from Europe, two from China and
Japan, and three were from Australia and New Zealand. Among the European included studies, the range (and seasonal reporting) of $25(\mathrm{OH}) \mathrm{D}$ varied between the countries. The lowest concentration of $25(\mathrm{OH}) \mathrm{D}$ reported was 17.8 (16.619.1) $\mathrm{nmol} / \mathrm{l}$ in Austria, while the highest concertation reported were 79.2 (74.4-84.0) $\mathrm{nmol} / 1$ in Italy and $81.7(52.1-111.3) \mathrm{nmol} / \mathrm{l}$ is Spain. Overall, the highest $25(\mathrm{OH}) \mathrm{D}$ concentration was reported in community-dwelling participants from the USA $82(74.6-89.4) \mathrm{nmol} / 1$ which is located at a latitude of $42^{\circ} \mathrm{N}$. The lowest concentration was reported in institutionalized from Austria at a latitude $48^{\circ} \mathrm{N}$. Using the $25 \mathrm{nmol} / 1$ as threshold to define vitamin D deficiency ( 8 of the 18 studies), the highest prevalence of deficiency $(33 \%)$ was found in Newcastle (UK), which is located at latitude $55^{\circ} \mathrm{N}$, while the lowest prevalence of deficiency $(2 \%)$ was found in New Zealand at latitude $38^{\circ} \mathrm{S}$.

Discussion: Substantial proportions of very old adults across the globe have poor vitamin D status which is exacerbated by extremes of latitude and institutionalization.

Conclusion: $25(\mathrm{OH}) \mathrm{D}$ concentrations in very old adults vary significantly by latitude and living condition.

\section{Abstract ID: 82 \\ Original Research \\ Subcategory: Fracture risk}

The impact of the secular increase in body mass index of hip fracture risk in the Norwegian population

\section{HK Kjeldgaard ', K Holvik', M O'Flaherty², GS Tell ${ }^{3}, H E$ Meyer ${ }^{1,4}$}

'Department of Chronic Diseases and Ageing, Norwegian Institute of Public Health, Oslo, Norway, ${ }^{2}$ Department of Public Health \& Policy, University of Liverpool, Liverpool, UK, ${ }^{3}$ Department of Global Public Health and Primary Care, University of Bergen, Bergen, Norway, 4Department of Community Medicine and Global Health, University of Oslo, Oslo, Norway

Background: A low body mass index (BMI: weight $(\mathrm{kg}) /$ height $(\mathrm{m}) 2$ ) is an important risk factor for hip fracture. In Norway, hip fracture rates have declined in recent decades at the same time as BMI has increased in the population; however, to what extent increased BMI has contributed to the declining hip fracture trend is unknown. We aimed to estimate the number of incident hip fractures prevented or postponed 
(IFPP) attributable to increased BMI using an adaptation of IMPACT coronary heart disease model methods.

Objective: To quantify the contribution of population BMI change over time to the observed decline in hip fracture rates in Norway between 1999 and 2018.

Methods: Hip fractures in Norway from 1999 to 2018 were extracted from the Norwegian hip fracture database (NORHip) and mean BMI of the Norwegian population was extrapolated from the fourth wave (1994-1995) and the seventh wave (2015-2016) of the Tromsø study. We calculated the difference between the observed number of hip fractures in 2018 and the expected number given stable fracture rates. IFPPs in 2018 attributable to changes in mean BMI were estimated according to sex and 5-year age groups from 50 to $85+$.

Results: Hip fracture rates decreased by $27 \%$ with 2,468 fewer hip fractures observed than expected in 2018. The mean BMI had increased in all age groups in both men and women over the time period by about $5 \%$. Unadjusted estimates showed that the increased mean BMI accounted for $\sim 35 \%$ of the decline, that is, 852 IFPPs.

Discussion: These preliminary results suggest that the increase in population mean BMI, reflecting a shift in the population BMI distribution, has significantly contributed to the declining hip fracture incidence.

Conclusion: The incident hip fracture rates in Norway declined between 1999 and 2018. These preliminary results suggest that increased $\mathrm{BMI}$ in the population has been a significant contributor to this decline.

\section{Abstract ID: 83 \\ Original Research \\ Subcategory: Management and treatment}

\section{*Monitoring osteoporosis in primary care: PINP and osteoporosis in Sheffield evaluation (pose study)}

\author{
L Mattia', C Mark-Wagstaff', B Abrahamsen², \\ S Davis ${ }^{3}, N$ Peel'4, R Eastell', M Schini ${ }^{1}$ \\ ${ }^{1}$ Department of Oncology and Metabolism, University of Sheffield, \\ Sheffield, UK, 'Department of Medicine, University of Southern Denmark,
}

Odense, Denmark, ${ }^{3}$ School of Health and Related Research, University of Sheffield, Sheffield, UK, 4Metabolic Bone Centre, Sheffield Teaching Hospitals NHS FT, Sheffield, UK

Background: The s-PINP (serum procollagen type I N propeptide) measurement was introduced into our service in 2011 and we have provided a monitoring algorithm for the management of osteoporosis treatment delivered in primary care.

Objective: Our aims were to evaluate whether PINP monitoring was associated with improved treatment initiation, less likelihood of a gap in treatment, better bone health outcomes (BMD increase, fracture risk), and more likely change treatment in a real-world setting.

Methods: We performed an observational study in 1406 patients. The inclusion criteria were referral from GPs, BMD scan performed in our department between 01/01/2012 and 31/12/2013 and PINP measurement performed in our laboratory (Roche Cobas autoanalyzer, Penzberg, Germany). Treatment initiation was recommended in BMD reports sent to the GP. The population was divided into Group A (intention to monitor, with baseline PINP) and Group B (no intention to monitor, without baseline PINP). Clinical, radiological and biochemical data have been retrospectively extracted. We report an interim analysis of 681 patients.

Results: Within the cohort of patients (mean age 71.3 years, $81 \%$ female), we found no statistical differences in baseline characteristics between two groups (age, gender, BMI, BMD and major risk factors). More patients in group A $(n=350,79 \%)$ started oral treatment than in group B $(n=116,49 \%)(p<0.001)$. There were no differences in the presence of gap (more than 3 months between two prescriptions) between two groups. More patients who had follow-up BMD scan (at 4-6 years from baseline) belonged to Group A (46\%) than B (29\%), $p<0.001$. During follow-up, patients in Group A had a greater increase of hip BMD $(+2.6 \%)$ than $\mathrm{B}$ $(+0.2 \%) \quad(p \quad 0.009)$ and were more likely to change management ( $p$ 0.037). The number of new fractures was less in Group A but was not statistically significant.

Discussion: Patients monitored with PINP are more likely to start oral treatment, have follow-up DXA scans, have a greater increase of hip BMD and change management. 
Conclusion: PINP monitoring can be initiated in general practice and is associated with improved clinical management.

\section{Abstract ID: 86 \\ Original Research \\ Subcategory: Vertebral fracture}

\section{Computer-aided opportunistic identification of vertebral fragility fractures in computed tomography images}

\section{PA Bromiley 1 , EP Kariki², TF Cootes ${ }^{1}$}

1 Division of Informatics, Imaging and Data Sciences, University of Manchester, Manchester, UK, ${ }^{2}$ Radiology, Manchester Academic Health Science Centre, Manchester University NHS Foundation Trust, Manchester, UK

Objective: Osteoporosis is a common, progressive skeletal disorder and is associated with considerable increases in morbidity and mortality. Vertebral fractures (VFs) are often the earliest clinical manifestation and indicate a significantly increased risk of future fractures. However, VFs are under-reported in clinical practice, particularly when incidentally visualised in computed tomography (CT) images. A recent Royal College of Radiologists (RCR) audit revealed that such fractures were accurately reported in only $26.2 \%$ of patients, and appropriate onward referral was recommended in only $2.6 \% .{ }^{1}$

Methods: We have created a computer-aided diagnostic system for VFs, using machine learning software to automatically identify vertebral bodies in CT images and classify fractures. The software can localise vertebral outlines with sub$\mathrm{mm}$ accuracy, enabling automation of standard vertebral body height measurements and fracture grading. It forms the basis of a commercial osteoporosis case-finding service designed to increase incidental VF reporting rates in CT images. This operates as a tele-radiology service that runs in parallel with hospital-based radiology reporting. Diagnoses are reviewed by in-house radiologists to ensure accuracy and patient safety, and a VF-specific report is then automatically generated and returned to the requesting hospital and the patient's local Fracture Liaison Service (FLS).

Results: Three prospective feasibility studies of the service were conducted at NHS hospitals, covering 12 months of CT imaging for patients aged over 50 (9797 patients; $50.1 \%$ female). The service reported VFs in $20.6 \%$ of patients $(50.3 \%$ female); $33.0 \%$ of these were also reported by radiologists at the requesting hospitals but only $5.2 \%$ had been referred to their local FLS, similar to the reporting and referral rates found in the RCR audit. ${ }^{1}$ As a result, the service referred 1944 patients for further management.

Discussion and Conclusion: The studies have not evaluated the accuracy of the service itself; this will require a future multi-reader study. However, we conclude that the service can identify significantly more VFs than hospital-based reporting, and that its automation of FLS referral may be an equally important route to patient benefit.

\section{References}

1. Howlett DC, Drinkwater KJ, Mahmood N, et al. Radiology reporting of osteoporotic vertebral fragility fractures on computed tomography studies: results of a UK national audit. Eur Radiol 2020; 30: 4713-4723.

\section{Abstract ID: 88 \\ Original Research \\ Subcategory: Fracture risk \\ Neuromuscular function as a contributor to fracture risk in type 1 diabetes}

T Vilaca, M Paggiosi, J Walsh, D Selvarajah, $R$ Eastell

Oncology and Metabolism, The University of Sheffield, Sheffield, UK

Background: Fracture risk is increased in people with diabetes and the risk is higher in those with longer and more severe disease. The underlying mechanisms are under investigation but extraskeletal features might be involved.

Objective: To assess muscle mass and strength and physical performance in people with type 1 diabetes with and without neuropathy and healthy controls.

Methods: We assessed appendicular skeletal muscle mass by dual-energy X-ray absorptiometry, handgrip strength using a digital hand dynamometer and physical performance by short physical performance battery (SPPB) in patients with type 1 diabetes with (T1DN+, $n=20)$ and without (T1DN-, $n=20$ ) distal symmetric polyneuropathy and healthy controls $(n=20)$. Appendicular skeletal muscle mass was normalized by height ${ }^{2}$ to 
calculate the appendicular skeletal muscle mass index. For the SPPB, participants were asked to stand with their feet side-by-side, in semi-tandem and tandem position, to stand up and sit down five times as quickly as possible, and to "walk at their usual speed" on an 8-foot walking course. Each of these were scored from 0 to 4 .

Results: T1DN+ had worse performance in the SPPB than T1DN- and controls. T1DN + scored 10.3 (2.1); T1DN- 11.8 (0.4) and controls 11.9 (0.4) $p<0.001$; four T1DN+ participants had poor performance (SPPB score $\leqslant 8$ ); Handgrip strength in $\mathrm{kg}[\mathrm{T} 1 \mathrm{DN}+28.5$ (20.6-33.9); T1DN- 28.4 (21.1-40.8) and controls 35.1 $(20.9-42.6) p<0.418]$ and appendicular muscular mass in $\mathrm{kg} / \mathrm{m}^{2}$ [T1DN + 7.4 (1.3); T1DN- 7.6 (1.2) and controls 7.4 (1.4) p0.853] were not different between the three groups.

Discussion: Despite no difference in appendicular muscle mass or handgrip strength, participants with diabetes and neuropathy scored lower in the SPPB. Four out of 20 had a poor performance, suggesting sarcopenia. Neuropathy is associated with an increased risk of falls and affects the lower more than the upper limbs. In addition, poor muscular function might have negative impact in bone.

Conclusion: Our findings suggest that neuromuscular dysfunction, as shown by poor physical performance, might contribute to the increase in the risk of fractures in people with type 1 diabetes and neuropathy.

\section{Abstract ID: 92 \\ Original Research \\ Subcategory: MSK}

Cone and non-cone total knee revisions: bone mineral density changes in the ipsilateral and contralateral hips

\section{Gundry', K Knapp', A Toms², P Hourigan², S Hopkins ${ }^{1}$ \\ ${ }^{1}$ Medical Imaging, University of Exeter Medical School, Exeter, UK, 2Princess Elizabeth Orthopaedic Centre, Royal Devon and Exeter Hospital, Exeter, UK}

Background: The association between total knee revisions (rTKR) and post-operative bone mineral density (BMD) changes in the hip is not well known. Furthermore, the impact of cone implantation in knee revisions and its impact on BMD in the ipsilateral and contralateral hips is less known.

Objective: This research investigated the potential impact of traditional rTKR and cone implantation rTKR on ipsilateral and contralateral hip BMD via dual energy X-ray absorptiometry (DXA) scans.

Method: 26 patients underwent a rTKR, 18 had a cone (Stryker) implant and eight had a noncone rTKR. Participants underwent bilateral hip DXA scans at pre-op (within 5 weeks of surgery), 6 weeks post-op, 3, 6 and 12 months post-op (20 completed 12 months, although some data were excluded due to total hip replacements). Participant's data were compared to their original

\section{Changes in the Ipsilateral hip BMD (\%) compared to pre-op, in cone and non-cone groups}

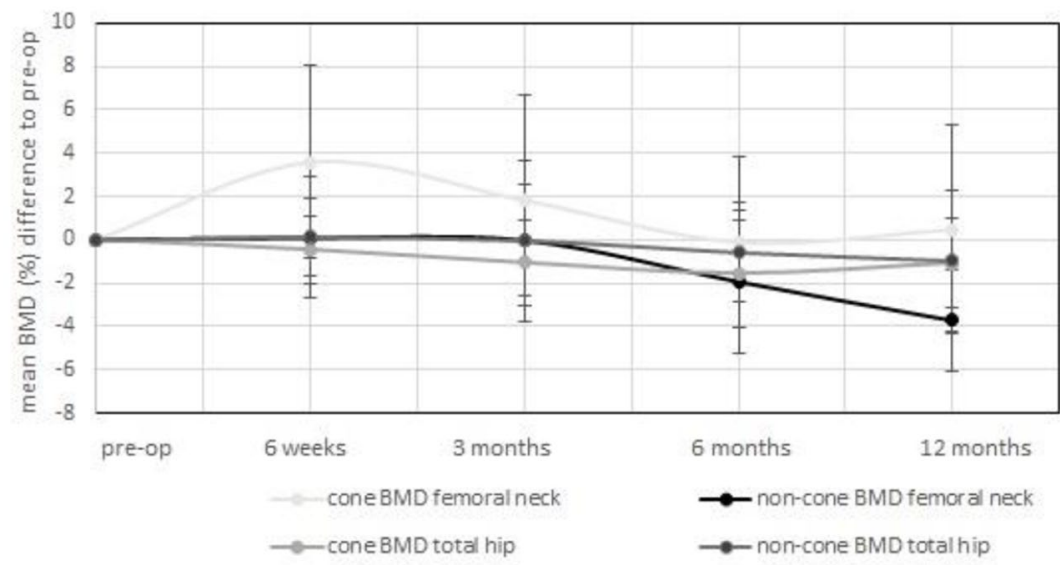

Figure 1. 
pre-op hip BMD across five regions of the hip (neck, wards, trochanter, shaft, total), with the $\mathrm{BMD}$ converted into a percentage change.

Results: Results are shown in Figure 1of the ipsilateral hip in both the cone and non-cone group.

Discussion: Both groups showed an increase in BMD at the femoral neck in the ipsilateral hip following rTKR. In the cone group this change is statistically significant at 6 weeks; this may be due to the high amount of trabecular bone in this region and the return to weight bearing activities. Although this increase is temporary, with the future visits demonstrating reduced increases or gradual losses.

Conclusion: rTKR patients from both groups show an increase in the femoral neck of the ipsilateral hip 6 weeks post-surgery, but this increase starts to decline across these regions throughout the following visits $\mathrm{rTKR}$ has a negative impact on hip BMD at 12 months.
Visit SAGE journals online journals.sagepub.com/ home/tab

(s)SAGE journals 


\section{Author index}

Abdy, S. 90

Abrahamsen, B. 83

Adams, E. 38

Aga, R. $65^{\star}$

Agyapong-Badu, S. 79

Ahmed, T. 24

Allen, MA. 9

Allsopp, PJ. 36

Almilaji, O. 49

Alshakh, RN. 16*, 39

Andrews, TM. 10

Anyan-Brown, SA. 94

Armstrong, DJ. 36

Arya, S. $76^{\star}$

Aspray, T. 68, 75, 80, 81, 90

Atkinson, K. 40

Ayis, S. 49

Azzabi, A. 75

Barbary, R. 89*

Barker, KL. 15

Barnes, H. 89

Bebbington, H. 97

Beevor, CB. 9

Beirne, A. 100

Bennett, A. 75

Ben-Shlomo, Y. 59

Berbecila, D. $84^{\star}$

Bevilacqua, GB. 22*, 25

Bishop, NJ. 71

Black, A. 91

Blagden, M. 8

Blair, L. 1

Blesic, K. 97

Boucher, BB. 25

Bradley, J. 95, 99

Bradley, S. 46

Broadbent, R. 6

Bromiley, PA. $86^{\star}$

Brown, M. 56

Bullock, L. 37, 45*, 47

Bunning, TB. 11, 18

Burns, A. 75

Burston, A. 66, 67

Butler, RB. 23

Byravan, S. $98^{\star}$

Carter, SA. 32, 62*

Cernovschi-Feasey, N. 73*

Chan, C. 97

Chandler, R. 75

Chastin, SFM. 79

Clark, E. 45, 47, 56, 66, 67
Clark, TC. $9^{\star}$

Clifton, L. 10

Clynes, MA. 7, 17, 22, 25, 33, 43* $44^{\star}$

Cold, S. 30

Collier, E. 2

Collins, GS. 10

Comben, E. 98

Connacher, S. 48, 69, 93

Cooles, F. 68

Cooper, CC. 7, 17, 22, 25, 32, 33, 43,

$44,62,71$

Cooper, L. 5

Cootes, TF. 86

Cordner, J. 46

Coyle, L. 46

Cracknell, A. 28

Crawford-Manning, F. 37 38,78

Crozier, SR. 62, 71

Cunningham, M. 40

Curtis, EM. $71^{\star}$

D'angelo, S. 43, 71

Dahl, C. 63

Dale, N. $38^{\star}$

Darling, AL. 19* $29^{\star}$

Darnell, G. 34, 97

Davda, D. 98

Davis, S. 66, 67, 83

Day, SD. 9

De Lusignan, S. 19

Dempster, R. 46

Dennison, EM. 7, 17, 22, 25, 32, 33,

43, 44, 62

Dockery, F. 100

Dodd, J. 96

Dolan, AL. 31, $77^{\star}$

Drinkwater, K. 57

Duncan, RD. 80

Dunstan, DW. 79

Dwivedi, R. 53, $74^{\star}$

Eastell, R. 29, 30, 71, 83, 88

Eckert, R. 48, 69, $93^{\star}$

Edwards, MHE. 9

Ellingsen, CL. 58

English, S. 46

Evans, SF. 12

Fagan, LF. 11

Feehan, O. $36^{\star}$

Ferguson, J. 75

Finnegan, MF. 9

Finnes, TE. 58

Fleming, J. 45, 47, 97
Folkestad, L. 30

Fox, P. 100

Frölich, J. 30

Fraser, R. 71

Fraser, WD. 79

French, MW. 13

Frew, J. 75

Frighi, V. $10^{\star}$

Fuggle, NR. $7^{\star}, 17^{\star}$

Fujita, F. 27

Fujita, M. 44

Fuller, A. 10

Fuller, N. 56

Gandhi, S. 71

Gendi, N. 39

George, S. 96

Gibson, MDJ. $31^{\star}$

Gilbert, FHA. 56*

Godfrey, KM. 62, 71

Gooberman-Hill, R. 66, 67

Gopal-Kothandapani, JS. 71

Gorman, P. 97

Gossiel, F. 29, 30

Goubar, A. $49^{\star}$

Gould, K. 38, $78^{\star}$

Grady, Cl. 42*

Greenall, C. 37

Gregson, CL. 24, 59

Greig, CA. 79

Griffin, JGL. 5, 40*, 50*, 51, 52, 57

Grimnes, G. 58

Gundry, M. $92^{\star}$

Gunnes, N. 61

Haeri, V. 48

Hakeem, SH. $80^{\star}, 81^{\star}$

Hamilton, LH. 9

Hannaway, N. $75^{\star}$

Hannink, E. $15^{\star}$

Hardcastle, S. 24

Hart, D. 24

Harvey, NC. 44, 62, 71

Havelock, W. 41

Hawarden, A. 37, 45

Hawley, SH. 11, 18

Heaton, CM. $2^{\star}$

Heijmans, S. 8

Hill, TH. 30, 80, 81

Hinchliffe, T. 94

Hjellvik, V. 61

Ho, SH. 23

Hoff, M. 61 


\section{Hoggett, NH. 9}

Hollick, R. 91

Holroyd, CR. 62

Holt, TA. 10

Holvik, K. 58*, 61* , 63, 65, 82

Hopkins, S. 92

Hourigan, P. 92

Howlett, DC. $57^{\star}$

Iglesias, C. 45, 47

Iki, M. 27

Illes, J. 57

Inman, DI. 11, 18

Inskip, HM. 62, 71

Iqbal, WI. 81

Jaalkhorol, M. 27*

Jagger, CJ. 80

Jamal, MS. $24^{\star}$

Jameson, KA. 7, 17, 22, 25

Javaid, MK. $28^{\star}, 44,48,57,69,71,93$

Jiang, X. 75

Jinks, C. 37, 45, 47

Johansen, A. 41

Johansen, AJ. 18*, 11*

Jones, GD. 49

Jones, O. $85^{\star}$

Judge, A. 59

Judge, AJ. 11, 18

Kagamimori, S. 27

Kajita, E. 27

Kanis, JA. 44

Kariki, EP. 86

Karlstad, Ø. 61

Kelly, J. 48, 69, 93

Kennedy, S. 71

Khalid, H. 94

Khera, T. $66^{\star}, 67^{\star}$

Kingston, AK. 80

Kjeldgaard, HK. 63, 82*

Knapp, KM. 56, 91, 92

Knell, J. 51*, 52*

Komatsu, M. 27

Kouda, K. 27

Lanham-New, SA. 29

Laskou, FL. 25*

Le, T. 87

Leigh, J. 2

Leong, A. 96

Leslie, WD. 44

Lewis, AR. 84

Lewis, K. 85

Leyland, S. 37

Lindsay, JR. $46^{\star}$

Lis, D. $87^{\star}$

Litwic, A. 32

Liz Fagan, LF. 18
Lloyd, M. 73

Low, R. 48, 69, 93

Magee, PJ. 36

Mahmood, N. 57

Mahoney, S. 48, 69, 93

Mallen, C. 45, 47

Malpass, T. 38, 78

Manning-Crawford, F. 45, 47

Mansour, R. 93

Mark-Wagstaff, C. 83

Marshall, L. 84

Martin, F. 49, 87, 94

Mathers, J. 30, 80

Mattia, L. $83^{\star}$

Mavroeidi, A. 42, 79*

Mccloskey, EV. 8*

Mcgregor, J. Mclorinan, JE. 35^

Mcmenemin, R. 75

Mcmichan, L. 79

Mcsorley, EM. 36

Md Yusof, MY. 35

Mendonça, NM. 81

Mendonca, NM. 80

Meyer, HE. 58, 61, 63* ${ }^{\star}$ 65, 82

Middleton, BA. 29

Mighton, S. 40

Milan, C. 48, 69, 93

Mony, D. 56

Moon, RJ. 71

Morgan, HS. 17

Morrin, H. 94*

Muir, D. $41^{\star}$

Muirhead, F. 42

Murday-Bailey, RMD. 9

Nadesalingam, K. 35

Nadkarni, V. 87

Namiraa, D. 27

Nandagudi, A. 16

Naylor, S. 99

Nelson, DA. 13*

Newman, M. 15

Nic Uidhir, A. 100

Noonan, KN. 25

O'kelly, J. 8

O'neill, T. 45, 47

O'flaherty, M. 82

Olajobi, O. 95

Oliver, S. 90

Onuekwa, S. 95*

Osborne, P. 48, 69, 93

Osterdahl, M. 87

Owen, N. 79

Paggiosi, M. 88

Palmer, K. 8

Pang, W. 98
Papageorghiou, A. 71

Papapoulos, SE. 8

Paskins, Z. 37, 38, 45, 47, 66, 67, 78

Patel, R. 59*

Pearson, R. 75

Pedley, I. 75

Peel, N. 83

Penketh, JP. 9

Perri, G. 30*

Peters, TJ. 66, 67

Pettit, R. 40

Phair, A. 40

Pletts, S. 60

Poon, MH. 84

Potter, C. 49

Pourshahidi, LK. 36

Powell, DE. 12* 23

Powrie, N. $5^{\star}, 6^{\star}, 14^{\star}$

Prentice, A. 71

Prieto Alhambra, D. 48, 69, 93

Pritt, AP. 9

Protheroe, J. 37

Quazi, S. 4

Rakieh, C. 12, 23*

Ramdewor, R. 89

Rathi, J. 8

Reynolds, A. 99*

Rice, G. 28

Richards, C. 14

Roast, J. 10

Roberts, B. $1^{\star}$

Roberts, F. 87

Roberts, TR. 23

Robertson, LAL. 13

Rodgers-Mansfield, BRM. 9

Ruiz-Martin, CR. 80

Søgaard, AJ. 58, 63, 65

Sackley, C. 49

Sadanandan, N. 54

Sahota, O. 54

Salem, K. 54

Salt, K. 96*

Salter, T. 73

Sapkota, H. 76, 96, 98, 99

Sayed, M. 53*

Schei, B. 63

Schini, M. 83

Schoenmakers, I. 71

Selvarajah, D. 88

Shahab, R. 87

Sheehan, KJ. 49

Shipley, J. 24

Showler, H. 75

Shuhart, CR. 44

Silva, SS. 23 
Skelton, DA. 42, 79

Skene, DJ. 29

Slevin, MM. 36

Smith, D. $91^{\star}$

Smith, MC. 10

Smitten, S. 76, 95, 99

Solbakken, SM. 58

Spice, C. 28

Spicer, SS. 9

Stad, RK. 8

Statham, LA. $68^{\star}, 90^{\star}$

Stevens, P. $48^{\star}, 69,93$

Stewart, S. 1

Stokes, O. 56

Sularz, AK. $34^{\star}$

Summers, R. 97

Syed, H. 4

Syed, L. $4^{\star}$

Tachiki, T. 27

Talsnes, O. 58

Tamaki, J. 27
Tang, JCY. 79

Tarawneh, A. $54^{\star}$

Tell, GS. 58, 61, 63, 82

Tharp, K. 97

Thorpe, O. $100^{*}$

Tian-Clarke, M. 38, 78

Titcombe, P. 17

Tobias, JH. 66, 67

Tobin, G. 90

Toms, A. 92

Tuck, S. 60

Turk, A. 41

Twemlow, A. 38

Venkatachalam, S. 76, 95, 96, 98, 99

Vilaca, T. $88^{\star}$

Vindlacheruvu, M. 34, 97

Walker-Bone, KE. 43

Walsh, J. 30, 88

Ward, K. 32, 62

Warren, S. 24

Watkins, E. 94
Watson, T. 75

Watts, H. 60

Webb, J. 24

Weerasuriya, NK. 84

Westbury, LD. 32, 44

White, G. 48, 69, 93

Whittleworth, R. 40

Wiles, B. 28

Williamson, T. 2

Wilton, W. 46

Winther, KH. 30

Woodhall, V. 68, 90

Wright, CW. 91

Wynn, K. 97

Young, M. 97

Young-Min, SAY. 9

Yura, A. 27

Zeb, S. 73

Zhang, J. 22, 32*, 33* 